\title{
Bell-Type Quantum Field Theories
}

\author{
Detlef Dürr, Sheldon Goldstein†, \\ Roderich Tumulka; and Nino Zanghi ${ }^{\S}$ \\ July 15, 2004
}

\begin{abstract}
In 3] John S. Bell proposed how to associate particle trajectories with a lattice quantum field theory, yielding what can be regarded as a $|\Psi|^{2}$-distributed Markov process on the appropriate configuration space. A similar process can be defined in the continuum, for more or less any regularized quantum field theory; such processes we call Bell-type quantum field theories. We describe methods for explicitly constructing these processes. These concern, in addition to the definition of the Markov processes, the efficient calculation of jump rates, how to obtain the process from the processes corresponding to the free and interaction Hamiltonian alone, and how to obtain the free process from the free Hamiltonian or, alternatively, from the one-particle process by a construction analogous to "second quantization." As an example, we consider the process for a second quantized Dirac field in an external electromagnetic field.
\end{abstract}

PACS numbers: 03.65.Ta, 02.50.-r, 03.70.+k

\section{Contents}

\begin{tabular}{lll}
\hline 1 & Introduction & 3
\end{tabular}

2 Ingredients of Bell-Type Quantum Field Theories 3

2.1 Review of Bohmian Mechanics and Equivariance . . . . . . . . . . . . . . 3

2.2 Equivariant Markov Processes . . . . . . . . . . . . . . . . . . . 4

2.3 Equivariant Jump Processes . . . . . . . . . . . . . . . . . . . . 6

*Mathematisches Institut der Universität München, Theresienstraße 39, 80333 München, Germany. E-mail: duerr@mathematik.uni-muenchen.de

$\dagger$ Departments of Mathematics and Physics - Hill Center, Rutgers, The State University of New Jersey, 110 Frelinghuysen Road, Piscataway, NJ 08854-8019, USA. E-mail: oldstein@math.rutgers.edu

${ }_{\ddagger}^{\ddagger}$ Dipartimento di Fisica and INFN sezione di Genova, Via Dodecaneso 33, 16146 Genova, Italy. E-mail: tumulka@mathematik.uni-muenchen.de

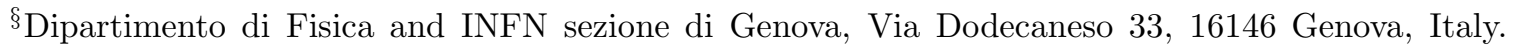
E-mail: zanghi@ge.infn.it 
2.4 Process Additivity . . . . . . . . . . . . . . . . . . . . . . . 7

2.5 What Added Processes Mav Look Like ....... . . . . . . . . . . . 8

2.6 Integral Operators Correspond to Jump Processed . . . . . . . . . . . . . 9

2.7 Minimal Jump Rates . . . . . . . . . . . . . . . . . . . . . . . . . . 10

2.8 Process Associated with the Free Hamiltonian . . . . . . . . . . . . 13

2.9 Other Approaches to the Free Process . . . . . . . . . . . . . . . . . 15

2.10 Bell-Tvpe QFT . . . . . . . . . . . . . . . . . . . . . . 17

2.11 More on Identical Particles . . . . . . . . . . . . . . . . . . . . . . . 18

3 Application to Simple Models $\quad 19$

3.1 A Simple QFT . . . . . . . . . . . . . . . . . . . . . . . . . . . . . . 19

3.2 Efficient Calculation of Rates in the Previous Example . . . . . . . . . 22

$3.2 .1 \quad$ A Diagram Notation . . . . . . . . . . . . . . . . . . 22

3.2 .2 Operations With Diagrams. . . . . . . . . . . . . . . . 24

3.2.3 Diagrams of Creation and Annihilation Operators . . . . . . . . 24

3.2.4 Application of the Diagram Method . . . . . . . . . . . . . . . . . 25

3.3 Pair Creation in an External Field . . . . . . . . . . . . . . . 26

3.3 .1 Fock Space and Hamiltonian . . . . . . . . . . . . . . . 27

3.3 .2 The Associated Process . . . . . . . . . . . . . . . . . . . . . . . 28

4 Second Quantization of a Markov Process 31

4.1 Preliminaries Concerning the Conditional Density Matrix . . . . . . . . . 31

4.2 Algorithm . . . . . . . . . . . . . . . . . . . . . . . . . . 33

4.2 .1 Direct Sums . . . . . . . . . . . . . . . . . . . . . 34

4.2 .2 Tensor Products . . . . . . . . . . . . . . . . . . . 34

4.2 .3 Second Quantization of the POVM . . . . . . . . . . . 35

4.2 .4 Construction of the Free Process $\ldots \ldots \ldots$. . . . . . . . 36

5 Towards a Notion of Minimal Process 36

$5.1 \quad$ Free Process From Differential Operators . . . . . . . . . . . . . . . 36

5.2 Minimalitv . . . . . . . . . . . . . . . . . . . . . . . 38

5.3 Minimal Processes $\ldots \ldots \ldots$. . . . . . . . . . . . . . . . 40

$\begin{array}{lll}6 & \text { Remarks } & 42\end{array}$

6.1 Svmmetries . . . . . . . . . . . . . . . . . . . . . . . 42

6.2 On the Notion of Reversibility . . . . . . . . . . . . . . . . . . . . 44

6.3 Heisenberg Picture . . . . . . . . . . . . . . . . . . . . . . 45

6.4 Examples of Process Additivitv . . . . . . . . . . . . . . . . . . 45

6.5 Second Quantization of a Minimal Jump Process . . . . . . . . . . . . 47

6.6 Global Existence Question . . . . . . . . . . . . . . . . . . . . . . . . . . 48

6.7 POVM Versus PVM . . . . . . . . . . . . . . . . . . . . . . . . . . 48

6.8 The Role of Field Operators . . . . . . . . . . . . . . . . . . . . . . . . . 49

$\begin{array}{lll}7 \text { Conclusions } & 50\end{array}$ 


\section{Introduction}

The aim of this paper is to present methods for constructing Bell-type QFTs. The primary variables of Bell-type QFTs are the positions of the particles. Bell suggested a dynamical law, governing the motion of the particles, in which the Hamiltonian $H$ and the state vector $\Psi$ determine certain jump rates 3 . Since these rates are in a sense the smallest choice possible, we call them the minimal jump rates. By construction, they preserve the $|\Psi|^{2}$ distribution. We assume a well-defined Hamiltonian as given; to achieve this, it is often necessary to introduce cut-offs. We shall assume this has been done where needed. In cases in which one has to choose between several possible position observables, for example because of issues related to the Newton-Wigner operator [27. 23. we shall also assume that a choice has been made.

Bell-type QFTs can also be regarded as extensions of Bohmian mechanics. When one tries to incorporate particle creation and annihilation into Bohmian mechanics, one is naturally lead to models like the one we presented in [14. The quantum equilibrium distribution, playing a central role in Bohmian mechanics, then more or less dictates that creation of a particle occurs in a stochastic manner-just as in Bell's model.

Bell-type QFTs have in common a good deal of mathematical structure, which we will elucidate. The paper is organized as follows. In Section 2 we introduce all the main ideas and reasonings; a superficial reading should focus on this section. Some examples of Bell-type QFTs are presented in Section 3. (Simple examples of minimal jump rates can be found in [15.) In Section 4 we describe the construction of a process for the free Hamiltonian based on "second quantization." In Section 5 we sketch the concept of the "minimal process" associated with a Hamiltonian $H$. Section 6 concerns some properties of Bell-type QFTs that derive from the construction methods developed in this paper. In Section 7 we conclude.

\section{Ingredients of Bell-Type Quantum Field Theories}

\subsection{Review of Bohmian Mechanics and Equivariance}

Bohmian mechanics [6, 19, 21] is a non-relativistic theory about $N$ point particles moving in 3-space, according to which the configuration $Q=\left(\boldsymbol{Q}_{1}, \ldots, \boldsymbol{Q}_{N}\right)$ evolves according to $^{1}$

$$
\frac{d Q}{d t}=v(Q), \quad v=\hbar \operatorname{Im} \frac{\Psi^{*} \nabla \Psi}{\Psi^{*} \Psi} .
$$

$\Psi=\Psi_{t}(q)$ is the wave function, which evolves according to the Schrödinger equation

$$
i \hbar \frac{\partial \Psi}{\partial t}=H \Psi
$$

\footnotetext{
${ }^{1}$ The masses $m_{k}$ of the particles have been absorbed in the Riemann metric $g_{\mu \nu}$ on configuration space $\mathbb{R}^{3 N}, g_{i a, j b}=m_{i} \delta_{i j} \delta_{a b}, i, j=1 \ldots N, a, b=1,2,3$, and $\nabla$ is the gradient associated with $g_{\mu \nu}$, i.e., $\nabla=\left(m_{1}^{-1} \nabla_{\boldsymbol{q}_{1}}, \ldots, m_{N}^{-1} \nabla_{\boldsymbol{q}_{N}}\right)$.
} 
with

$$
H=-\frac{\hbar^{2}}{2} \Delta+V
$$

for spinless particles, with $\Delta=\operatorname{div} \nabla$. For particles with spin, $\Psi$ takes values in the appropriate spin space $\mathbb{C}^{k}, V$ may be matrix valued, and numerator and denominator of (11) have to be understood as involving inner products in spin space. The secret of the success of Bohmian mechanics in yielding the predictions of standard quantum mechanics is the fact that the configuration $Q_{t}$ is $\left|\Psi_{t}\right|^{2}$-distributed in configuration space at all times $t$, provided that the initial configuration $Q_{0}$ (part of the Cauchy data of the theory) is so distributed. This property, called equivariance in [19, suffices for empirical agreement between any quantum theory (such as a QFT) and any version thereof with additional (often called "hidden") variables $Q$, provided the outcomes of all experiments are registered or recorded in these variables. That is why equivariance will be our guide for obtaining the dynamics of the particles.

The equivariance of Bohmian mechanics follows immediately from comparing the continuity equation for a probability distribution $\rho$ associated with (11),

$$
\frac{\partial \rho}{\partial t}=-\operatorname{div}(\rho v)
$$

with the equation satisfied by $|\Psi|^{2}$ which follows from (2),

$$
\frac{\partial|\Psi|^{2}}{\partial t}(q, t)=\frac{2}{\hbar} \operatorname{Im}\left[\Psi^{*}(q, t)(H \Psi)(q, t)\right] \text {. }
$$

In fact, it follows from (3) that

$$
\frac{2}{\hbar} \operatorname{Im}\left[\Psi^{*}(q, t)(H \Psi)(q, t)\right]=-\operatorname{div}\left[\hbar \operatorname{Im} \Psi^{*}(q, t) \nabla \Psi(q, t)\right]
$$

so, recalling (11), one obtains that

$$
\frac{\partial|\Psi|^{2}}{\partial t}=-\operatorname{div}\left(|\Psi|^{2} v\right)
$$

and hence that if $\rho_{t}=\left|\Psi_{t}\right|^{2}$ at some time $t$ then $\rho_{t}=\left|\Psi_{t}\right|^{2}$ for all times. Equivariance is an expression of the compatibility between the Schrödinger evolution for the wave function and the law, such as (11), governing the motion of the actual configuration. In [19, in which we were concerned only with the Bohmian dynamics (11), we spoke of the distribution $|\Psi|^{2}$ as being equivariant. Here we wish to find processes for which we have equivariance, and we shall therefore speak of equivariant processes and motions.

\subsection{Equivariant Markov Processes}

The study of example QFTs like that of [14] has lead us to the consideration of Markov processes as candidates for the equivariant motion of the configuration $Q$ for Hamiltonians $H$ more general than those of the form (3). 
Consider a Markov process $Q_{t}$ on configuration space. The transition probabilities are characterized by the backward generator $L_{t}$, a (time-dependent) linear operator acting on functions $f$ on configuration space:

$$
L_{t} f(q)=\frac{d}{d s} \mathbb{E}\left(f\left(Q_{t+s}\right) \mid Q_{t}=q\right)
$$

where $d / d s$ means the right derivative at $s=0$ and $\mathbb{E}(\cdot \mid \cdot)$ denotes the conditional expectation. Equivalently, the transition probabilities are characterized by the forward generator $\mathscr{L}_{t}$ (or, as we shall simply say, generator), which is also a linear operator but acts on (signed) measures on the configuration space. Its defining property is that for every process $Q_{t}$ with the given transition probabilities, the distribution $\rho_{t}$ of $Q_{t}$ evolves according to

$$
\frac{\partial \rho_{t}}{\partial t}=\mathscr{L}_{t} \rho_{t}
$$

$\mathscr{L}_{t}$ is the dual of $L_{t}$ in the sense that

$$
\int f(q) \mathscr{L}_{t} \rho(d q)=\int L_{t} f(q) \rho(d q)
$$

We will use both $L_{t}$ and $\mathscr{L}_{t}$, whichever is more convenient. We will encounter several examples of generators in the subsequent sections.

We can easily extend the notion of equivariance from deterministic to Markov processes. Given the Markov transition probabilities, we say that the $|\Psi|^{2}$ distribution is equivariant if and only if for all times $t$ and $t^{\prime}$ with $t<t^{\prime}$, a configuration $Q_{t}$ with distribution $\left|\Psi_{t}\right|^{2}$ evolves, according to the transition probabilities, into a configuration $Q_{t^{\prime}}$ with distribution $\left|\Psi_{t^{\prime}}\right|^{2}$. In this case, we also simply say that the transition probabilities are equivariant, without explicitly mentioning $|\Psi|^{2}$. Equivariance is equivalent to

$$
\mathscr{L}_{t}\left|\Psi_{t}\right|^{2}=\frac{\partial\left|\Psi_{t}\right|^{2}}{\partial t}
$$

for all $t$. When (11) holds (for a fixed $t$ ) we also say that $\mathscr{L}_{t}$ is an equivariant generator (with respect to $\Psi_{t}$ and $H$ ). Note that this definition of equivariance agrees with the previous meaning for deterministic processes.

We call a Markov process $Q$ equivariant if and only if for every $t$ the distribution $\rho_{t}$ of $Q_{t}$ equals $\left|\Psi_{t}\right|^{2}$. For this to be the case, equivariant transition probabilities are necessary but not sufficient. (While for a Markov process $Q$ to have equivariant transition probabilities amounts to the property that if $\rho_{t}=\left|\Psi_{t}\right|^{2}$ for one time $t$, where $\rho_{t}$ denotes the distribution of $Q_{t}$, then $\rho_{t^{\prime}}=\left|\Psi_{t^{\prime}}\right|^{2}$ for every $t^{\prime}>t$, according to our definition of an equivariant Markov process, in fact $\rho_{t}=\left|\Psi_{t}\right|^{2}$ for all $t$.) However, for equivariant transition probabilities there exists a unique equivariant Markov process.

The crucial idea for our construction of an equivariant Markov process is to note that (5) is completely general, and to find a generator $\mathscr{L}_{t}$ such that the right hand side of (15) can be read as the action of $\mathscr{L}$ on $\rho=|\Psi|^{2}$,

$$
\frac{2}{\hbar} \operatorname{Im} \Psi^{*} H \Psi=\mathscr{L}|\Psi|^{2} .
$$


We shall implement this idea beginning in Section 2.6, after a review of jump processes and some general considerations. But first we shall illustrate the idea with the familiar case of Bohmian mechanics.

For $H$ of the form (3), we have (6) and hence that

$$
\frac{2}{\hbar} \operatorname{Im} \Psi^{*} H \Psi=-\operatorname{div}\left(\hbar \operatorname{Im} \Psi^{*} \nabla \Psi\right)=-\operatorname{div}\left(|\Psi|^{2} \hbar \operatorname{Im} \frac{\Psi^{*} \nabla \Psi}{|\Psi|^{2}}\right) .
$$

Since the generator of the (deterministic) Markov process corresponding to the dynamical system $d Q / d t=v(Q)$ given by a velocity vector field $v$ is

$$
\mathscr{L} \rho=-\operatorname{div}(\rho v)
$$

we may recognize the last term of (13) as $\mathscr{L}|\Psi|^{2}$ with $\mathscr{L}$ the generator of the deterministic process defined by (11). Thus, as is well known, Bohmian mechanics arises as the natural equivariant process on configuration space associated with $H$ and $\Psi$.

To be sure, Bohmian mechanics is not the only solution of (12) for $H$ given by (3). Among the alternatives are Nelson's stochastic mechanics [26] and other velocity formulas [12. However, Bohmian mechanics is the most natural choice, the one most likely to be relevant to physics. It is, in fact, the canonical choice, in the sense of minimal process which we shall explain in Section 5.3 .

\subsection{Equivariant Jump Processes}

Let $\mathcal{Q}$ denote the configuration space of the process, whatever sort of space that may be (vector space, lattice, manifold, etc.); mathematically speaking, we need that $\mathcal{Q}$ be a measurable space. A (pure) jump process is a Markov process on $\mathcal{Q}$ for which the only motion that occurs is via jumps. Given that $Q_{t}=q$, the probability for a jump to $q^{\prime}$, i.e., into the infinitesimal volume $d q^{\prime}$ about $q^{\prime}$, by time $t+d t$ is $\sigma_{t}\left(d q^{\prime} \mid q\right) d t$, where $\sigma$ is called the jump rate. In this notation, $\sigma$ is a finite measure in the first variable; $\sigma(B \mid q)$ is the rate (the probability per unit time) of jumping to somewhere in the set $B \subseteq \mathcal{Q}$, given that the present location is $q$. The overall jump rate is $\sigma(\mathcal{Q} \mid q)$.

It is often the case that $\mathcal{Q}$ is equipped with a distinguished measure, which we shall denote by $d q$ or $d q^{\prime}$, slightly abusing notation. For example, if $\mathcal{Q}=\mathbb{R}^{d}, d q$ may be the Lebesgue measure, or if $\mathcal{Q}$ is a Riemannian manifold, $d q$ may be the Riemannian volume element. When $\sigma(\cdot \mid q)$ is absolutely continuous relative to the distinguished measure, we also write $\sigma\left(q^{\prime} \mid q\right) d q^{\prime}$ instead of $\sigma\left(d q^{\prime} \mid q\right)$. Similarly, we sometimes use the letter $\rho$ for denoting a measure and sometimes the density of a measure, $\rho(d q)=\rho(q) d q$.

A jump first occurs when a random waiting time $T$ has elapsed, after the time $t_{0}$ at which the process was started or at which the most recent previous jump has occurred. For purposes of simulating or constructing the process, the destination $q^{\prime}$ can be chosen at the time of jumping, $t_{0}+T$, with probability distribution $\sigma_{t_{0}+T}(\mathcal{Q} \mid q)^{-1} \sigma_{t_{0}+T}(\cdot \mid q)$. In case the overall jump rate is time-independent, $T$ is exponentially distributed with mean $\sigma(\mathcal{Q} \mid q)^{-1}$. When the rates are time-dependent - as they will typically be in what 
follows - the waiting time remains such that

$$
\int_{t_{0}}^{t_{0}+T} \sigma_{t}(\mathcal{Q} \mid q) d t
$$

is exponentially distributed with mean 1, i.e., $T$ becomes exponential after a suitable (time-dependent) rescaling of time. For more details about jump processes, see [8].

The generator of a pure jump process can be expressed in terms of the rates:

$$
\mathscr{L}_{\sigma} \rho(d q)=\int_{q^{\prime} \in \mathcal{Q}}\left(\sigma\left(d q \mid q^{\prime}\right) \rho\left(d q^{\prime}\right)-\sigma\left(d q^{\prime} \mid q\right) \rho(d q)\right),
$$

a "balance" or "master" equation expressing $\partial \rho / \partial t$ as the gain due to jumps to $d q$ minus the loss due to jumps away from $q$.

We shall say that jump rates $\sigma$ are equivariant if $\mathscr{L}_{\sigma}$ is an equivariant generator. It is one of our goals in this paper to describe a general scheme for obtaining equivariant jump rates. In Sections [2.6 and 2.7] we will explain how this leads us to formula (29).

\subsection{Process Additivity}

The Hamiltonian of a QFT usually comes as a sum, such as

$$
H=H_{0}+H_{I}
$$

with $H_{0}$ the free Hamiltonian and $H_{I}$ the interaction Hamiltonian. If several particle species are involved, $H_{0}$ is itself a sum containing one free Hamiltonian for each species. The left hand side of (12), which should govern our choice of the generator, is then also a sum,

$$
\frac{2}{\hbar} \operatorname{Im} \Psi^{*} H_{0} \Psi+\frac{2}{\hbar} \operatorname{Im} \Psi^{*} H_{I} \Psi=\mathscr{L}|\Psi|^{2}
$$

This opens the possibility of finding a generator $\mathscr{L}$ by setting $\mathscr{L}=\mathscr{L}_{0}+\mathscr{L}_{I}$, provided we have generators $\mathscr{L}_{0}$ and $\mathscr{L}_{I}$ corresponding to $H_{0}$ and $H_{I}$ in the sense that

$$
\begin{aligned}
& \frac{2}{\hbar} \operatorname{Im} \Psi^{*} H_{0} \Psi=\mathscr{L}_{0}|\Psi|^{2} \\
& \frac{2}{\hbar} \operatorname{Im} \Psi^{*} H_{I} \Psi=\mathscr{L}_{I}|\Psi|^{2} .
\end{aligned}
$$

This feature of (12) we call process additivity; it is based on the fact that the left hand side of (12) is linear in $H$. Note that the backward generator of the process with forward generator $\mathscr{L}_{0}+\mathscr{L}_{I}$ is $L_{0}+L_{I}$; thus forward and backward generators lead to the same notion of process additivity, and to the same process corresponding to $H_{0}+H_{I}$. In many cases, as will be elaborated in Section 2.8. $H_{0}$ is based on an operator known from quantum mechanics (e.g., the Dirac operator), in such a way that $\mathscr{L}_{0}$ can be obtained from the appropriate Bohmian law of motion. In Section 2.6 we will explain how $\mathscr{L}_{I}$ can usually be taken as the generator of a jump process. 
Our proposal is to take seriously the process generated by $\mathscr{L}=\mathscr{L}_{0}+\mathscr{L}_{I}$ and regard it as the process naturally associated with $H$. The bottom line is that process additivity provides a method of constructing a Bell-type theory.

Obviously, the mathematical observation of process additivity (that sums of generators define an equivariant process associated with sums of Hamiltonians) applies not only to the splitting of $H$ into a free and an interaction contribution, but to every case where $H$ is a sum. And it seems that process additivity provides a physically very reasonable process in every case where $H$ is naturally a sum, in fact the most reasonable process: the one that should be considered the Bell-type process, defining the Bell-type theory.

\subsection{What Added Processes May Look Like}

To get some feeling for what addition of generators, $\mathscr{L}=\mathscr{L}_{1}+\mathscr{L}_{2}$, means for the corresponding processes, we consider some examples. First consider two deterministic processes (on the same configuration space), having generators of the form $\mathscr{L} \rho=-\operatorname{div}(\rho v)$. To add the generators obviously means to add the velocity vector fields, $v=v_{1}+v_{2}$, so the resulting velocity is a superposition of two contributions.

Next consider a pure jump process. Since, according to (15), the generator $\mathscr{L}$ is linear in $\sigma$, adding generators means adding rates, $\sigma=\sigma_{1}+\sigma_{2}$. This is equivalent to saying there are two kinds of jumps: if the present location is $q \in \mathcal{Q}$, with probability $\sigma_{1}(\mathcal{Q} \mid q) d t$ the process performs a jump of the first type within the next $d t$ time units, and with probability $\sigma_{2}(\mathcal{Q} \mid q) d t$ a jump of the second type. That does not mean, however, that one can decide from a given realization of the process which jump was of which type.

Next suppose we add the generators of a deterministic and a jump process,

$$
\mathscr{L} \rho(q)=-\operatorname{div}(\rho v)(q)+\int_{q^{\prime} \in \mathcal{Q}}\left(\sigma\left(q \mid q^{\prime}\right) \rho\left(q^{\prime}\right)-\sigma\left(q^{\prime} \mid q\right) \rho(q)\right) d q^{\prime} .
$$

This process moves with velocity $v(q)$ until it jumps to $q^{\prime}$, where it continues moving, with velocity $v\left(q^{\prime}\right)$. The jump rate may vary with time in two ways: first because $\sigma$ may be time-dependent, second because $\sigma$ may be position-dependent and $Q_{t}$ moves with velocity $v$. One can easily understand (19) in terms of gain or loss of probability density due to motion and jumps. So this process is piecewise deterministic: although the temporal length of the pieces (the intervals between two subsequent jumps) and the starting points (the jump destinations) are random, given this data the trajectory is determined.

The generator of the Wiener process in $\mathbb{R}^{d}$ is the Laplacian, and to add to it the generator of a deterministic process means to introduce a drift. Note that this is different from adding, in $\mathbb{R}^{d}$, a Wiener process to a solution of the deterministic process. In spaces like $\mathbb{R}^{d}$, where it so happens that one is allowed to add locations, there is a danger of confusing addition of generators with addition of realizations. Whenever we speak of adding processes, it means we add generators. 
To add generators of a diffusion and a pure jump process yields what is often called a jump diffusion process, one making jumps with time- and position-dependent rates and following a diffusion path in between. Diffusion processes, however, will play almost no role in this paper.

\subsection{Integral Operators Correspond to Jump Processes}

We now address the interaction part $H_{I}$ of the Hamiltonian (16). In QFTs with cutoffs it is usually the case that $H_{I}$ is an integral operator. For that reason, we shall in this work focus on integral operators for $H_{I}$. We now point out why the naturally associated process is a pure jump process. For short, we will write $H$ rather than $H_{I}$ in this and the subsequent section. For the time being, think of $\mathcal{Q}$ as $\mathbb{R}^{d}$ and of wave functions as complex valued.

What characterizes jump processes versus continuous processes is that some amount of probability that vanishes at $q \in \mathcal{Q}$ can reappear in an entirely different region of configuration space, say at $q^{\prime} \in \mathcal{Q}$. This is manifest in the equation for $\partial \rho / \partial t$, (15)): the first term in the integrand is the probability increase due to arriving jumps, the second the decrease due to departing jumps, and the integration over $q^{\prime}$ reflects that $q^{\prime}$ can be anywhere in $\mathcal{Q}$. This suggests that Hamiltonians for which the expression (15) for $\partial|\Psi|^{2} / \partial t$ is naturally an integral over $d q^{\prime}$ correspond to pure jump processes. So when is the left hand side of (12) an integral over $d q^{\prime}$ ? When $H$ is an integral operator, i.e., when $\left\langle q|H| q^{\prime}\right\rangle$ is not merely a formal symbol, but represents an integral kernel that exists as a function or a measure and satisfies

$$
(H \Psi)(q)=\int d q^{\prime}\left\langle q|H| q^{\prime}\right\rangle \Psi\left(q^{\prime}\right) .
$$

In this case, we should choose the jump rates in such a way that, when $\rho=|\Psi|^{2}$,

$$
\sigma\left(q \mid q^{\prime}\right) \rho\left(q^{\prime}\right)-\sigma\left(q^{\prime} \mid q\right) \rho(q)=\frac{2}{\hbar} \operatorname{Im} \Psi^{*}(q)\left\langle q|H| q^{\prime}\right\rangle \Psi\left(q^{\prime}\right),
$$

and this suggests, since jump rates must be nonnegative (and the right hand side of (21) is anti-symmetric), that

$$
\sigma\left(q \mid q^{\prime}\right) \rho\left(q^{\prime}\right)=\left[\frac{2}{\hbar} \operatorname{Im} \Psi^{*}(q)\left\langle q|H| q^{\prime}\right\rangle \Psi\left(q^{\prime}\right)\right]^{+}
$$

(where $x^{+}$denotes the positive part of $x \in \mathbb{R}$, that is, $x^{+}$is equal to $x$ for $x>0$ and is zero otherwise), or

$$
\sigma\left(q \mid q^{\prime}\right)=\frac{\left[(2 / \hbar) \operatorname{Im} \Psi^{*}(q)\left\langle q|H| q^{\prime}\right\rangle \Psi\left(q^{\prime}\right)\right]^{+}}{\Psi^{*}\left(q^{\prime}\right) \Psi\left(q^{\prime}\right)} .
$$

These rates are an instance of what we call the minimal jump rates associated with $H$ (and $\Psi$ ). The name comes from the fact that they are actually the minimal possible 
values given (21), as is expressed by the inequality (114) and will be explained in detail in Section 5.2. Minimality entails that at any time $t$, one of the transitions $q_{1} \rightarrow q_{2}$ or $q_{2} \rightarrow q_{1}$ is forbidden. We will call the process defined by the minimal jump rates the minimal jump process (associated with $H$ ).

In contrast to jump processes, continuous motion, as in Bohmian mechanics, corresponds to such Hamiltonians that the formal matrix elements $\left\langle q|H| q^{\prime}\right\rangle$ are nonzero only infinitesimally close to the diagonal, and in particular to differential operators like the Schrödinger Hamiltonian (3), which has matrix elements of the type $\delta^{\prime \prime}\left(q-q^{\prime}\right)+V(q) \delta\left(q-q^{\prime}\right)$. We can summarize the situation, as a rule of thumb, by the following table:

\begin{tabular}{|r|l|}
\hline A contribution to $H$ that is a $\ldots$ & corresponds to ... \\
\hline integral operator & jumps \\
differential operator & deterministic continuous motion \\
multiplication operator & no motion $(\mathscr{L}=0)$ \\
\hline
\end{tabular}

The minimal jump rates as given by (22) have some nice features. The possible jumps for this process correspond to the nonvanishing matrix elements of $H$ (though, depending on the state $\Psi$, even some of the jump rates corresponding to nonvanishing matrix elements of $H$ might happen to vanish). Moreover, in their dependence on the state $\Psi$, the jump rates $\sigma$ depend only "locally" upon $\Psi$ : the jump rate for a given jump $q^{\prime} \rightarrow q$ depends only on the values $\Psi\left(q^{\prime}\right)$ and $\Psi(q)$ corresponding to the configurations linked by that jump. Discretizing $\mathbb{R}^{3}$ to a lattice $\varepsilon \mathbb{Z}^{3}$, one can obtain Bohmian mechanics as a limit $\varepsilon \rightarrow 0$ of minimal jump processes [33, 34], whereas greater-than-minimal jump rates lead to Nelson's stochastic mechanics [26] and similar diffusions, such as (117); see [34, 22. If the Schrödinger operator (3) is approximated in other ways by operators corresponding to jump processes, e.g., by $H_{\varepsilon}=e^{-\varepsilon H} H e^{-\varepsilon H}$, the minimal jump processes presumably also converge to Bohmian mechanics.

We have reason to believe that there are lots of self-adjoint operators which do not correspond to any stochastic process that can be regarded as defined, in any reasonable sense, by (22). ${ }^{2}$ But such operators seem never to occur in QFT. (The Klein-Gordon operator $\sqrt{m^{2} c^{4}-\hbar^{2} c^{2} \Delta}$ does seem to have a process, but it requires a more detailed discussion which will be provided in a forthcoming work [18.)

\subsection{Minimal Jump Rates}

The reasoning of the previous section applies to a far more general setting than just considered: to arbitrary configuration spaces $\mathcal{Q}$ and "generalized observables" - POVMsdefining, for our purposes, what the "position representation" is. We now present this more general reasoning, which leads to one of the main formulas of this paper, (29).

\footnotetext{
${ }^{2}$ Consider, for example, $H=p \cos p$ where $p$ is the one-dimensional momentum operator $-i \hbar \partial / \partial q$. Its formal kernel $\left\langle q|H| q^{\prime}\right\rangle$ is the distribution $-\frac{i}{2} \delta^{\prime}\left(q-q^{\prime}-1\right)-\frac{i}{2} \delta^{\prime}\left(q-q^{\prime}+1\right)$, for which (22) would not have a meaning. From a sequence of smooth functions converging to this distribution, one can obtain a sequence of jump processes with rates (22): the jumps occur very frequently, and are by amounts of approximately \pm 1 . A limiting process, however, does not exist.
} 
The process we construct relies on the following ingredients from QFT:

1. A Hilbert space $\mathscr{H}$ with scalar product $\langle\Psi \mid \Phi\rangle$.

2. A unitary one-parameter group $U_{t}$ in $\mathscr{H}$ with Hamiltonian $H$,

$$
U_{t}=e^{-\frac{i}{\hbar} t H},
$$

so that in the Schrödinger picture the state $\Psi$ evolves according to

$$
i \hbar \frac{d \Psi_{t}}{d t}=H \Psi_{t}
$$

$U_{t}$ could be part of a representation of the Poincaré group.

3. A positive-operator-valued measure (POVM) $P(d q)$ on $\mathcal{Q}$ acting on $\mathscr{H}$, so that the probability that the system in the state $\Psi$ is localized in $d q$ at time $t$ is

$$
\mathbb{P}_{t}(d q)=\left\langle\Psi_{t}|P(d q)| \Psi_{t}\right\rangle
$$

Mathematically, a POVM $P$ on $\mathcal{Q}$ is a countably additive set function ("measure"), defined on measurable subsets of $\mathcal{Q}$, with values in the positive (bounded self-adjoint) operators on (a Hilbert space) $\mathscr{H}$, such that $P(\mathcal{Q})$ is the identity operator. ${ }^{3}$ Physically, for our purposes, $P(\cdot)$ represents the (generalized) position observable, with values in $\mathcal{Q}$. The notion of POVM generalizes the more familiar situation of observables given by a set of commuting self-adjoint operators, corresponding, by means of the spectral theorem, to a projection-valued measure (PVM): the case where the positive operators are projection operators. A typical example is the single Dirac particle: the position operators on $L^{2}\left(\mathbb{R}^{3}, \mathbb{C}^{4}\right)$ induce there a natural PVM $P_{0}(\cdot)$ : for any Borel set $B \subseteq$ $\mathbb{R}^{3}, P_{0}(B)$ is the projection to the subspace of functions that vanish outside $B$, or, equivalently, $P_{0}(B) \Psi(q)=\mathbf{1}_{B}(q) \Psi(q)$ with $\mathbf{1}_{B}$ the indicator function of the set $B$. Thus, $\left\langle\Psi\left|P_{0}(d q)\right| \Psi\right\rangle=|\Psi(q)|^{2} d q$. When one considers as Hilbert space $\mathscr{H}$ only the subspace of positive energy states, however, the localization probability is given by $P(\cdot)=P_{+} P_{0}(\cdot) I$ with $P_{+}: L^{2}\left(\mathbb{R}^{3}, \mathbb{C}^{4}\right) \rightarrow \mathscr{H}$ the projection and $I: \mathscr{H} \rightarrow L^{2}\left(\mathbb{R}^{3}, \mathbb{C}^{4}\right)$ the inclusion mapping. Since $P_{+}$does not commute with most of the operators $P_{0}(B)$, $P(\cdot)$ is no longer a PVM but a genuine $\mathrm{POVM}^{4}$ and consequently does not correspond to any position operator - although it remains true (for $\Psi$ in the positive energy subspace) that $\langle\Psi|P(d q)| \Psi\rangle=|\Psi(q)|^{2} d q$. That is why in QFT, the position observable is indeed more often a POVM than a PVM. POVMs are also relevant to photons [1, 25]. In one approach, the photon wave function $\Psi: \mathbb{R}^{3} \rightarrow \mathbb{C}^{3}$ is subject to the constraint condition $\nabla \cdot \Psi=\partial_{1} \Psi_{1}+\partial_{2} \Psi_{2}+\partial_{3} \Psi_{3}=0$. Thus, the physical Hilbert space $\mathscr{H}$ is the (closure

\footnotetext{
${ }^{3}$ The countable additivity is to be understood as in the sense of the weak operator topology. This in fact implies that countable additivity also holds in the strong topology.

${ }^{4}$ This situation is indeed more general than it may seem. By a theorem of Naimark [11 p. 142], every POVM $P(\cdot)$ acting on $\mathscr{H}$ is of the form $P(\cdot)=P_{+} P_{0}(\cdot) P_{+}$where $P_{0}$ is a PVM on a larger Hilbert space, and $P_{+}$the projection to $\mathscr{H}$.
} 
of the) subspace of $L^{2}\left(\mathbb{R}^{3}, \mathbb{C}^{3}\right)$ defined by this constraint, and the natural PVM on $L^{2}\left(\mathbb{R}^{3}, \mathbb{C}^{3}\right)$ gives rise, by projection, to a POVM on $\mathscr{H}$. So much for POVMs. Let us get back to the construction of a jump process.

The goal is to specify equivariant jump rates $\sigma=\sigma^{\Psi, H, P}$, i.e., such rates that

$$
\mathscr{L}_{\sigma} \mathbb{P}=\frac{d \mathbb{P}}{d t} .
$$

To this end, one may take the following steps:

1. Note that

$$
\frac{d \mathbb{P}_{t}(d q)}{d t}=\frac{2}{\hbar} \operatorname{Im}\left\langle\Psi_{t}|P(d q) H| \Psi_{t}\right\rangle
$$

2. Insert the resolution of the identity $I=\int_{q^{\prime} \in \mathcal{Q}} P\left(d q^{\prime}\right)$ and obtain

$$
\frac{d \mathbb{P}_{t}(d q)}{d t}=\int_{q^{\prime} \in \mathcal{Q}} \mathbb{J}_{t}\left(d q, d q^{\prime}\right)
$$

where

$$
\mathbb{J}_{t}\left(d q, d q^{\prime}\right)=\frac{2}{\hbar} \operatorname{Im}\left\langle\Psi_{t}\left|P(d q) H P\left(d q^{\prime}\right)\right| \Psi_{t}\right\rangle .
$$

3. Observe that $\mathbb{J}$ is anti-symmetric, $\mathbb{J}\left(d q^{\prime}, d q\right)=-\mathbb{J}\left(d q, d q^{\prime}\right)$. Thus, since $x=x^{+}-$ $(-x)^{+}$,

$\mathbb{J}\left(d q, d q^{\prime}\right)=\left[(2 / \hbar) \operatorname{Im}\left\langle\Psi\left|P(d q) H P\left(d q^{\prime}\right)\right| \Psi\right\rangle\right]^{+}-\left[(2 / \hbar) \operatorname{Im}\left\langle\Psi\left|P\left(d q^{\prime}\right) H P(d q)\right| \Psi\right\rangle\right]^{+}$.

4. Multiply and divide both terms by $\mathbb{P}(\cdot)$, obtaining that

$$
\begin{aligned}
\int_{q^{\prime} \in \mathcal{Q}} \mathbb{J}\left(d q, d q^{\prime}\right)= & \int_{q^{\prime} \in \mathcal{Q}}\left(\frac{\left[(2 / \hbar) \operatorname{Im}\left\langle\Psi\left|P(d q) H P\left(d q^{\prime}\right)\right| \Psi\right\rangle\right]^{+}}{\left\langle\Psi\left|P\left(d q^{\prime}\right)\right| \Psi\right\rangle} \mathbb{P}\left(d q^{\prime}\right)-\right. \\
& \left.-\quad \frac{\left[(2 / \hbar) \operatorname{Im}\left\langle\Psi\left|P\left(d q^{\prime}\right) H P(d q)\right| \Psi\right\rangle\right]^{+}}{\langle\Psi|P(d q)| \Psi\rangle} \mathbb{P}(d q)\right) .
\end{aligned}
$$

5. By comparison with (15), recognize the right hand side of the above equation as $\mathscr{L}_{\sigma} \mathbb{P}$, with $\mathscr{L}_{\sigma}$ the generator of a Markov jump process with jump rates

$$
\sigma\left(d q \mid q^{\prime}\right)=\frac{\left[(2 / \hbar) \operatorname{Im}\left\langle\Psi\left|P(d q) H P\left(d q^{\prime}\right)\right| \Psi\right\rangle\right]^{+}}{\left\langle\Psi\left|P\left(d q^{\prime}\right)\right| \Psi\right\rangle}
$$

which we call the minimal jump rates. 
Mathematically, the right hand side of this formula as a function of $q^{\prime}$ must be understood as a density (Radon-Nikodým derivative) of one measure relative to another. ${ }^{5}$ The plus symbol denotes the positive part of a signed measure; it can also be understood as applying the plus function, $x^{+}=\max (x, 0)$, to the density, if it exists, of the numerator.

To sum up, we have argued that with $H$ and $\Psi$ is naturally associated a Markov jump process $Q_{t}$ whose marginal distributions coincide at all times by construction with the quantum probability measure, $\rho_{t}(\cdot)=\mathbb{P}_{t}(\cdot)$, so that $Q_{t}$ is an equivariant Markov process.

In Section 4 of [15], we establish precise conditions on $H, P$, and $\Psi$ under which the jump rates (29) are well-defined and finite $\mathbb{P}$-almost everywhere, and prove that in this case the rates are equivariant, as suggested by the steps 1-5 above. It is perhaps worth remarking at this point that any $H$ can be approximated by Hamiltonians $H_{n}$ (namely Hilbert-Schmidt operators) for which the rates (29) are always (for all $\Psi$ ) well-defined and equivariant [15]. Concerning this, see also the end of Section 5.3 .

\subsection{Process Associated with the Free Hamiltonian}

We now address the free Hamiltonian $H_{0}$ of a QFT. We describe the process naturally associated with $H_{0}$, when this is the second quantized Schrödinger or Dirac operator. We will treat more general free Hamiltonians in the next section. We shall consider here

\footnotetext{
${ }^{5}$ Quite aside from the previous discussion, it is perhaps worth noting that there are not so many expressions in $H, P$, and $\Psi$ that would meet the formal criteria for being a candidate for the jump rate. Since the only connection between abstract Hilbert space and configuration space is by $P$, which leads to measures on $\mathcal{Q}$, the only way to obtain a function on $\mathcal{Q}$ is to form a Radon-Nikodým quotient of two measures, $\sigma\left(q^{\prime}\right)=A\left(d q^{\prime}\right) / B\left(d q^{\prime}\right)$. Since $\sigma$ must be a measure-valued function, the numerator should be a bi-measure (a measure in each of two variables). The simplest measure one can form from $H, P$, and $\Psi$ is $\langle\Psi|P(d q)| \Psi\rangle$; the simplest bi-measures are $\left\langle\Psi\left|H^{n_{1}} P(d q) H^{n_{2}} P\left(d q^{\prime}\right) H^{n_{3}}\right| \Psi\right\rangle$. Jump rates must have dimension $1 /$ time, and the only object at hand having this dimension is $H / \hbar$. Thus, $H$ can appear only once in the numerator. The expressions $\left\langle\Psi\left|H P(d q) P\left(d q^{\prime}\right)\right| \Psi\right\rangle$ and $\left\langle\Psi\left|P(d q) P\left(d q^{\prime}\right) H\right| \Psi\right\rangle$ are no good because for PVMs $P$ they are concentrated on the diagonal of $\mathcal{Q} \times \mathcal{Q}$ and hence do not lead to nontrivial jumps. Let us write $\mu$ for the measure-valued function we have arrived at:$$
\mu\left(d q, q^{\prime}\right)=\frac{1}{\hbar} \frac{\left\langle\Psi\left|P(d q) H P\left(d q^{\prime}\right)\right| \Psi\right\rangle}{\left\langle\Psi\left|P\left(d q^{\prime}\right)\right| \Psi\right\rangle} .
$$

This provides complex measures, whereas $\sigma\left(\cdot \mid q^{\prime}\right)$ must be a positive real measure. There are not many ways of forming a positive real measure from a complex one, the essential ones being

$$
|\mu|,|\operatorname{Re} \mu|,|\operatorname{Im} \mu|,(\operatorname{Re} \mu)^{+},(\operatorname{Re} \mu)^{-},(\operatorname{Im} \mu)^{+},(\operatorname{Im} \mu)^{-}
$$

times a numerical constant $\lambda>0$. One could of course form additional expressions at the price of higher complexity.

This has gotten us already pretty close to the minimal rates (29), which correspond to $\sigma=2(\operatorname{Im} \mu)^{+}$. To proceed further, we might demand the absence of unnecessary jumps; that means that at any time, either the jump $q_{1} \rightarrow q_{2}$ or $q_{2} \rightarrow q_{1}$ is forbidden; this leaves only $\lambda(\operatorname{Im} \mu)^{ \pm}$. Moreover, $2(\operatorname{Im} \mu)^{+}$is the only expression in the list that has Bohmian mechanics as a limiting case or implies equivariance. Furthermore it corresponds to the natural guess (118) for a backward generator, discussed in Section 5.3
} 
only Hamiltonians for one type of particle.

We first define the configuration space $\mathcal{Q}$. Let us write $\mathcal{Q}^{(1)}$ ("one-particle configuration space") for physical space; this is typically, but not necessarily, $\mathbb{R}^{3}$. The space $\mathcal{Q}$ in which the "free process" takes place is the configuration space for a variable number of identical particles; we call it $\Gamma \mathcal{Q}^{(1)}$. It can be defined as the space of all finite subsets-with-multiplicities of $\mathcal{Q}^{(1)}$. A set-with-multiplicities consists of a set and, for each element $x$ of the set, a positive integer, called the multiplicity of $x$. The number of particles in a configuration $q$ is the sum of its multiplicities, \#q. Such configurations describe several identical particles, some of which may be located at the same position in space. Equivalently, one could say that $\Gamma \mathcal{Q}^{(1)}$ is the set of all mappings $n: \mathcal{Q}^{(1)} \rightarrow \mathbb{N} \cup\{0\}$ (meaning the number of particles at a given location) such that

$$
\sum_{\boldsymbol{q} \in \mathcal{Q}^{(1)}} n(\boldsymbol{q})<\infty
$$

Another equivalent definition is the set of all finite nonnegative measures $n(\cdot)$ on $\mathcal{Q}^{(1)}$ that assume only integer values; the meaning of $n(R)$ is the number of particles in the region $R$ of physical space. Finally, one can define

$$
\Gamma \mathcal{Q}^{(1)}=\bigcup_{n=0}^{\infty} \mathcal{Q}^{(n)} \text { where } \mathcal{Q}^{(n)}=\left(\mathcal{Q}^{(1)}\right)^{n} / \text { permutations. }
$$

A related space, for which we write $\Gamma_{\neq} \mathcal{Q}^{(1)}$, is the space of all finite subsets of $\mathcal{Q}^{(1)}$; it is contained in $\Gamma \mathcal{Q}^{(1)}$, after obvious identifications. In fact, $\Gamma_{\neq} \mathcal{Q}^{(1)}=\Gamma \mathcal{Q}^{(1)} \backslash \Delta$, where $\Delta$ is the set of coincidence configurations, i.e., those having two or more particles at the same position. $\Gamma_{\neq} \mathcal{Q}^{(1)}$ is the union of the spaces $\mathcal{Q}_{\neq}^{(n)}$ for $n=0,1,2, \ldots$, where $\mathcal{Q}_{\neq}^{(n)}$ is the space of subsets of $\mathcal{Q}^{(1)}$ with $n$ elements.

For $\mathcal{Q}^{(1)}=\mathbb{R}^{d}$, the $n$-particle sector $\mathcal{Q}_{\neq}^{(n)}$ is a manifold of dimension $n d$ (see [13] for a discussion of Bohmian mechanics on this manifold). If $d \geq 2$, the set $\Delta$ of coincidence configurations has codimension $\geq 2$ and thus can usually be ignored. We can then replace $\Gamma \mathbb{R}^{d}$ by the somewhat simpler space $\Gamma_{\neq} \mathbb{R}^{d}$.

The position POVM $P^{(1)}$ on $\mathcal{Q}^{(1)}$ (acting on the one-particle Hilbert space) naturally leads to a POVM we call $\Gamma P^{(1)}$ on $\mathcal{Q}=\Gamma \mathcal{Q}^{(1)}$, acting on Fock space (see Section 4.2 .3 for the definition). ${ }^{6}$ Since a configuration from $\Gamma\left(\mathbb{R}^{3}\right)$ defines the number of particles and their positions, the name "position observable" for $P=\Gamma P^{(1)}$ stretches the meaning of "position" somewhat: it now also encompasses the number of particles.

We now give a description of the free process associated with the second-quantized Schrödinger operator; it arises from Bohmian mechanics. Fock space $\mathscr{H}=\mathscr{F}$ is a direct sum

$$
\mathscr{F}=\bigoplus_{n=0}^{\infty} \mathscr{F}^{(n)}
$$

\footnotetext{
${ }^{6}$ The coincidence configurations form a null set, $\Gamma P^{(1)}(\Delta)=0$, when $\mathcal{Q}^{(1)}$ is a continuum, or, more precisely, when $P^{(1)}$ is nonatomic as a measure.
} 
where $\mathscr{F}^{(n)}$ is the $n$-particle Hilbert space. $\mathscr{F}^{(n)}$ is the subspace of symmetric (for bosons) or anti-symmetric (for fermions) functions in $L^{2}\left(\mathbb{R}^{3 n},\left(\mathbb{C}^{2 s+1}\right)^{\otimes n}\right)$ for spin- $s$ particles. Thus, $\Psi \in \mathscr{F}$ can be decomposed into a sequence $\Psi=\left(\Psi^{(0)}, \Psi^{(1)}, \ldots, \Psi^{(n)}, \ldots\right)$, the $n$-th member $\Psi^{(n)}$ being an $n$-particle wave function, the wave function representing the $n$-particle sector of the quantum state vector. The obvious way to obtain a process on $\mathcal{Q}=\Gamma \mathbb{R}^{3}$ is to let the configuration $Q(t)$, containing $N=\# Q(t)$ particles, move according to the $N$-particle version of Bohm's law (1), guided by $\Psi^{(N)} .^{7}$ This is indeed an equivariant process since $H_{0}$ has a block diagonal form with respect to the decomposition (301),

$$
H_{0}=\bigoplus_{n=0}^{\infty} H_{0}^{(n)}
$$

and $H_{0}^{(n)}$ is just a Schrödinger operator for $n$ noninteracting particles, for which, as we already know, Bohmian mechanics is equivariant. We used a very similar process in [14] (the only difference being that particles were numbered in [14]).

Similarly, if $H_{0}$ is the second quantized Dirac operator, we let a configuration $Q$ with $N$ particles move according to the usual $N$-particle Bohm-Dirac law [7, p. 274]

$$
\frac{d Q}{d t}=c \frac{\Psi^{*}(Q) \alpha_{N} \Psi(Q)}{\Psi^{*}(Q) \Psi(Q)}
$$

where $c$ denotes the speed of light and $\alpha_{N}=\left(\boldsymbol{\alpha}^{(1)}, \ldots, \boldsymbol{\alpha}^{(N)}\right)$ with $\boldsymbol{\alpha}^{(k)}$ acting on the spin index of the $k$-th particle.

\subsection{Other Approaches to the Free Process}

We will give below a general velocity formula, applicable to a wider class of free Hamiltonians. Alternatively, we can provide a free process for any $H_{0}$ if we are given an equivariant process for the one-particle Hamiltonian $H^{(1)}$. This is based on the particular mathematical structure of $H_{0}$, which can be expressed by saying it arises from a one-particle Hamiltonian $H^{(1)}$ by applying a "second quantization functor $\Gamma$ " [29]. That is, there is an algorithm (in a bosonic or fermionic version) for forming, from a one-particle Hilbert space $\mathscr{H}^{(1)}$ and a one-particle Hamiltonian $H^{(1)}$, a Fock space $\mathscr{F}=\Gamma \mathscr{H}^{(1)}$ and free Hamiltonian $H_{0}=\Gamma H^{(1)}$. And parallel to this "second quantization" algorithm, there is an algorithm for the canonical construction, from a given equivariant one-particle Markov process $Q_{t}^{(1)}$, of a process we call $\Gamma Q_{t}^{(1)}$ that takes place in $\mathcal{Q}=\Gamma \mathcal{Q}^{(1)}$ and is equivariant with respect to $H_{0}$. This algorithm may be called the "second quantization" of a Markov process.

The algorithm is described in Section 4.2. What the algorithm does is essentially to construct an $n$-particle version of $Q_{t}^{(1)}$ for every $n$, and finally combine these by means of a random particle number $N=N(t)=\# Q(t)$ which is constant under the free

\footnotetext{
${ }^{7}$ As defined, configurations are unordered, whereas we have written Bohm's law (11) for ordered configurations. Thanks to the (anti-)symmetry of the wave function, however, all orderings will lead to the same particle motion. For more about such considerations, see our forthcoming work [13].
} 
process, parallel to the fact that the particle number operator is conserved by $H_{0}$. We note further that the process $\Gamma Q_{t}^{(1)}$ is deterministic if $Q_{t}^{(1)}$ is. If we take the one-particle process to be Bohmian mechanics or the Bohm-Dirac motion, the algorithm reproduces the processes described in the previous section.

The algorithm leaves us with the task of finding a suitable one-particle law, which we do not address in this paper. For some Hamiltonians, such as the Dirac operator, this is immediate, for others it is rather nontrivial, or even unsolved. The Klein-Gordon operator $\sqrt{m^{2} c^{4}-\hbar^{2} c^{2} \Delta}$ will be discussed in forthcoming work [18, and for a study of photons see [28].

When $H_{0}$ is made of differential operators of up to second order (which includes of course the Schrödinger and Dirac operators), there is another way to characterize the process associated with $H_{0}$, a way which allows a particularly succinct description of the process and a particularly direct derivation and construction. In fact, we give a formula for its backward generator $L_{0}$, or alternatively the velocity (or the forward generator $\left.\mathscr{L}_{0}\right)$, in terms of $H_{0}, P$, and $\Psi$.

We begin by defining, for any $H, P$, and $\Psi$, an operator $L$ acting on functions $f: \mathcal{Q} \rightarrow \mathbb{R}$, which may or may not be the backward generator of a process, by

$$
L f(q)=\operatorname{Re} \frac{\langle\Psi|P(d q) \hat{L} \hat{f}| \Psi\rangle}{\langle\Psi|P(d q)| \Psi\rangle}=\operatorname{Re} \frac{\left\langle\Psi\left|P(d q) \frac{i}{\hbar}[H, \hat{f}]\right| \Psi\right\rangle}{\langle\Psi|P(d q)| \Psi\rangle} .
$$

where $[$,$] means the commutator,$

$$
\hat{f}=\int_{q \in \mathcal{Q}} f(q) P(d q)
$$

and $\hat{L}$ is the "generator" of the (Heisenberg) time evolution of the operator $\hat{f}$,

$$
\hat{L} \hat{f}=\left.\frac{d}{d \tau} e^{i H \tau / \hbar} \hat{f} e^{-i H \tau / \hbar}\right|_{\tau=0}=\frac{i}{\hbar}[H, \hat{f}] .
$$

(If $P$ is a PVM, then $\hat{f}=f(\hat{q})$, where $\hat{q}$ is the configuration operator.) (32) could be guessed in the following way: since $L f$ is in a certain sense, see (8) , the time derivative of $f$, it might be expected to be related to $\hat{L} \hat{f}$, which is in a certain sense, see (34), the time derivative of $\hat{f}$. As a way of turning the operator $\hat{L} \hat{f}$ into a function $L f(q)$, the middle term in (32) is an obvious possibility. Note that this way of arriving at (32) does not make use of equivariance; for another way that does, see Section [5.1]

The formula for the forward generator equivalent to (32) reads

$$
\mathscr{L} \rho(d q)=\operatorname{Re}\left\langle\Psi\left|\frac{\widehat{d \rho}}{d \mathbb{P}} \frac{i}{\hbar}[H, P(d q)]\right| \Psi\right\rangle
$$

as follows from (10).

Whenever $L$ is indeed a backward generator, we call it the minimal free (backward) generator associated with $\Psi, H$, and $P$. (The name is based on the concept of minimal process as explained in Section 5.3.) Then the corresponding process is equivariant (see 
Section 5.1). This is the case if (and, there is reason to expect, only if ) $P$ is a PVM and $H$ is a differential operator of up to second order in the position representation, in which $P$ is diagonal. In that case, the process is deterministic, and the backward generator has the form $L=v \cdot \nabla$ where $v$ is the velocity vector field; thus, (32) directly specifies the velocity, in the form of a first-order differential operator $v \cdot \nabla$. In case $H$ is the $N$-particle Schrödinger operator with or without spin, (32) yields the Bohmian velocity (11), and if $H$ is the Dirac operator, the Bohm-Dirac velocity (31). To sum up, in some cases definition (32) leads to just the right backward generator.

To return to our starting point: if the one-particle generator $\mathscr{L}^{(1)}$ arises from the one-particle Hamiltonian $H^{(1)}$ by (35), then (35) also holds between the free generator $\mathscr{L}_{0}=\Gamma \mathscr{L}^{(1)}$ and the free Hamiltonian $H_{0}=\Gamma H^{(1)}$. (See Section 5.1 for details.) In other words, (32) is compatible with the "second quantization" algorithm. Thus, in relevant cases (32) allows a direct definition of the free process in terms of $H_{0}$, just as (29) directly defines, in terms of $H_{I}$, the jump rates.

A relevant point is that the "second quantization" of a differential operator is again a differential operator, in a suitable sense, and has the same order. Note also that (32), when applied to the second quantized Schrödinger or Dirac Hamiltonian, defines the same vector field on $\Gamma\left(\mathbb{R}^{3}\right)$ as described in the previous section.

\subsection{Bell-Type QFT}

We briefly summarize what we have obtained. A Bell-type QFT is about particles moving in physical 3-space; their number and positions are represented by a point $Q_{t}$ in configuration space $\mathcal{Q}$. Provided physical space is $\mathbb{R}^{3}, \mathcal{Q}$ is usually $\Gamma \mathbb{R}^{3}$ or a Cartesian product of several such spaces, each factor representing a different particle species. $Q_{t}$ follows a Markov process in $\mathcal{Q}$, which is governed by a state vector $\Psi$ in a suitable Hilbert space $\mathscr{H}$. $\mathscr{H}$ is related to $\mathcal{Q}$ by means of a PVM or POVM $P$. $\Psi$ undergoes a unitary evolution with Hamiltonian $H$. The process $Q_{t}$ usually consists of deterministic continuous trajectories interrupted by stochastic jumps; more generally, it arises by process additivity (i.e., by adding generators) from a free process associated with $H_{0}$ and a jump process associated with $H_{I}$. The jump rates are given by (29) for $H=H_{I}$. The free process arises from Bohmian mechanics, or a suitable analogue, by a construction that can be formalized as the "second quantization" of a one-particle Markov process; when appropriate, it is defined directly by (32). The process $Q_{t}$ is equivariant, i.e., $\left\langle\Psi_{t}|P(d q)| \Psi_{t}\right\rangle$ distributed.

Examples of Bell-type QFTs can be found in [3, 14, and in Section 3, It is our contention that, essentially, there is a unique Bell-type version of every regularized QFT. We have to postpone, however, the discussion of operators of the Klein-Gordon type. We also have to assume that the QFT provides us with the POVM $P(\cdot)$; this is related to an ongoing discussion in the literature [27, 25, 23] concerning the right position operator. 


\subsection{More on Identical Particles}

The $n$-particle sector of the configuration space (without coincidence configurations) of identical particles $\Gamma_{\neq}\left(\mathbb{R}^{3}\right)$ is the manifold of $n$-point subsets of $\mathbb{R}^{3}$; let $\mathcal{Q}$ be this manifold. The most common way of describing the quantum state of $n$ fermions is by an anti-symmetric (square-integrable) wave function $\Psi$ on $\hat{\mathcal{Q}}:=\mathbb{R}^{3 n}$; let $\mathscr{H}$ be the space of such functions. Whereas for bosons $\Psi$ could be viewed as a function on $\mathcal{Q}$, for fermions $\Psi$ is not a function on $\mathcal{Q}$.

Nonetheless, the configuration observable still corresponds to a PVM $P$ on $\mathcal{Q}$ : for $B \subseteq \mathcal{Q}$, we set $P(B) \Psi\left(\boldsymbol{q}_{1}, \ldots, \boldsymbol{q}_{n}\right)=\Psi\left(\boldsymbol{q}_{1}, \ldots, \boldsymbol{q}_{n}\right)$ if $\left\{\boldsymbol{q}_{1}, \ldots, \boldsymbol{q}_{n}\right\} \in B$ and zero otherwise. In other words, $P(B)$ is multiplication by the indicator function of $\pi^{-1}(B)$ where $\pi$ is the obvious projection mapping $\hat{\mathcal{Q}} \backslash \Delta \rightarrow \mathcal{Q}$, with $\Delta$ the set of coincidence configurations.

To obtain other useful expressions for this PVM, we introduce the formal kets $|\hat{q}\rangle$ for $\hat{q} \in \hat{\mathcal{Q}}$ (to be treated like elements of $L^{2}(\hat{\mathcal{Q}})$ ), the anti-symmetrization operator $S$ (i.e., the projection $L^{2}(\hat{\mathcal{Q}}) \rightarrow \mathscr{H}$ ), the normalized anti-symmetrizer ${ }^{8} s=\sqrt{n !} S$, and the formal kets $|s \hat{q}\rangle:=s|\hat{q}\rangle$ (to be treated like elements of $\mathscr{H}$ ). The $|\hat{q}\rangle$ and $|s \hat{q}\rangle$ are normalized in the sense that

$$
\left\langle\hat{q} \mid \hat{q}^{\prime}\right\rangle=\delta\left(\hat{q}-\hat{q}^{\prime}\right) \text { and }\left\langle s \hat{q} \mid s \hat{q}^{\prime}\right\rangle=(-1)^{\varrho\left(\hat{q}, \hat{q}^{\prime}\right)} \delta\left(q-q^{\prime}\right),
$$

where $q=\pi(\hat{q}), q^{\prime}=\pi\left(\hat{q}^{\prime}\right), \varrho\left(\hat{q}, \hat{q}^{\prime}\right)$ is the permutation that carries $\hat{q}$ into $\hat{q}^{\prime}$ given that $q=q^{\prime}$, and $(-1)^{\varrho}$ is the sign of the permutation $\varrho$. Now we can write

$$
P(d q)=\sum_{\hat{q} \in \pi^{-1}(q)}|\hat{q}\rangle\langle\hat{q}|d q=n ! S| \hat{q}\rangle\langle\hat{q}|d q=| s \hat{q}\rangle\langle s \hat{q}| d q,
$$

where the sum is over the $n$ ! ways of numbering the $n$ points in $q$; the last two terms actually do not depend on the choice of $\hat{q} \in \pi^{-1}(q)$, the numbering of $q$.

The probability distribution arising from this PVM is

$$
\mathbb{P}(d q)=\sum_{\hat{q} \in \pi^{-1}(q)}|\Psi(\hat{q})|^{2} d q=n !|\Psi(\hat{q})|^{2} d q=|\langle s \hat{q} \mid \Psi\rangle|^{2} d q
$$

with arbitrary $\hat{q} \in \pi^{-1}(q)$.

There is a way of viewing fermion wave functions as being defined on $\mathcal{Q}$, rather than $\mathbb{R}^{3 n}$, by regarding them as cross-sections of a particular 1-dimensional vector bundle over $\mathcal{Q}$. To this end, define an $n$ !-dimensional vector bundle $E$ by

$$
E_{q}:=\bigoplus_{\hat{q} \in \pi^{-1}(q)} \mathbb{C} .
$$

${ }^{8}$ The name means this: since $S$ is a projection, $S \Psi$ is usually not a unit vector when $\Psi$ is. Whenever $\Psi \in L^{2}(\hat{\mathcal{Q}})$ is supported by a fundamental domain of the permutation group, i.e., by a set $\Omega \subseteq \hat{\mathcal{Q}}$ on which (the restriction of) $\pi$ is a bijection to $\mathcal{Q}$, the norm of $S \Psi$ is $1 / \sqrt{n !}$, so that $s \Psi$ is again a unit vector. 
Every function $\Psi: \mathbb{R}^{3 n} \rightarrow \mathbb{C}$ naturally gives rise to a cross-section $\Phi$ of $E$, defined by

$$
\Phi(q):=\bigoplus_{\hat{q} \in \pi^{-1}(q)} \Psi(\hat{q}) .
$$

The anti-symmetric functions form a 1-dimensional subbundle of $E$ (see also [13] for a discussion of this bundle).

\section{Application to Simple Models}

In this section, we point out how the jump rates of the model in [14] are contained in (29) and present a full-fledged Bell-type QFT for the second-quantized Dirac equation in an external electromagnetic field.

Further cut-off QFTs that may provide interesting examples of Bell-type QFTs, worth a detailed discussion in a future work [17, are the scalar self-interacting field (e.g., $\left.\Phi^{4}\right)$, QED, and other gauge field theories. We have to postpone the treatment of these theories because they require discussions lying outside the scope of this paper, in particular a discussion of the position representation of photon wave functions in QED, and, concerning $\Phi^{4}$, of the appropriate probability current for the Klein-Gordon equation.

\subsection{A Simple QFT}

We presented a simple example of a Bell-type QFT in [14, and we will now briefly point to the aspects of this model that are relevant here. The model is based on one of the simplest possible QFTs [32, p. 339].

The relevant configuration space $\mathcal{Q}$ for a QFT (with a single particle species) is the configuration space of a variable number of identical particles in $\mathbb{R}^{3}$, which is the set $\Gamma\left(\mathbb{R}^{3}\right)$, or, ignoring the coincidence configurations (as they are exceptions), the set $\Gamma_{\neq}\left(\mathbb{R}^{3}\right)$ of all finite subsets of $\mathbb{R}^{3}$. The $n$-particle sector of this is a manifold of dimension $3 n$; this configuration space is thus a union of (disjoint) manifolds of different dimensions. The relevant configuration space for a theory with several particle species is the Cartesian product of several copies of $\Gamma_{\neq}\left(\mathbb{R}^{3}\right)$. In the model of [14, there are two particle species, a fermion and a boson, and thus the configuration space is

$$
\mathcal{Q}=\Gamma_{\neq}\left(\mathbb{R}^{3}\right) \times \Gamma_{\neq}\left(\mathbb{R}^{3}\right) .
$$

We will denote configurations by $q=(x, y)$ with $x$ the configuration of the fermions and $y$ the configuration of the bosons.

For simplicity, we replaced in [14] the sectors of $\Gamma_{\neq}\left(\mathbb{R}^{3}\right) \times \Gamma_{\neq}\left(\mathbb{R}^{3}\right)$, which are manifolds, by vector spaces of the same dimension (by artificially numbering the particles), and obtained the union

$$
\hat{\mathcal{Q}}=\bigcup_{n=0}^{\infty}\left(\mathbb{R}^{3}\right)^{n} \times \bigcup_{m=0}^{\infty}\left(\mathbb{R}^{3}\right)^{m},
$$


with $n$ the number of fermions and $m$ the number of bosons. Here, however, we will use (40) as the configuration space, since we have already discussed the space $\Gamma_{\neq}\left(\mathbb{R}^{3}\right)$. In comparison with (41), this amounts to (merely) ignoring the numbering of the particles.

$\mathscr{H}$ is the tensor product of a fermion Fock space and a boson Fock space, and thus the subspace of wave functions in $L^{2}(\hat{\mathcal{Q}})$ that are anti-symmetric in the fermion coordinates and symmetric in the boson coordinates. Let $S$ denote the appropriate symmetrization operator, i.e., the projection operator $L^{2}(\hat{\mathcal{Q}}) \rightarrow \mathscr{H}$, and $s$ the normalized symmetrizer

$$
s \Psi\left(\boldsymbol{x}_{1}, \ldots, \boldsymbol{x}_{n}, \boldsymbol{y}_{1}, \ldots, \boldsymbol{y}_{m}\right)=\sqrt{n ! m !} S \Psi\left(\boldsymbol{x}_{1}, \ldots, \boldsymbol{x}_{n}, \boldsymbol{y}_{1}, \ldots, \boldsymbol{y}_{m}\right),
$$

i.e., $s=\sqrt{N ! M !} S$ with $N$ and $M$ the fermion and boson number operators, which commute with $S$ and with each other. As in Section 2.11] we denote by $\pi$ the projection mapping $\hat{\mathcal{Q}} \backslash \Delta \rightarrow \mathcal{Q}, \pi\left(\boldsymbol{x}_{1}, \ldots, \boldsymbol{x}_{n}, \boldsymbol{y}_{1}, \ldots, \boldsymbol{y}_{m}\right)=\left(\left\{\boldsymbol{x}_{1}, \ldots, \boldsymbol{x}_{n}\right\},\left\{\boldsymbol{y}_{1}, \ldots, \boldsymbol{y}_{m}\right\}\right)$. The configuration PVM $P(B)$ on $\mathcal{Q}$ is multiplication by $\mathbf{1}_{\pi^{-1}(B)}$, which can be understood as acting on $\mathscr{H}$, though it is defined on $L^{2}(\hat{\mathcal{Q}})$, since it is permutation invariant and thus maps $\mathscr{H}$ to itself. We utilize again the formal kets $|\hat{q}\rangle$ where $\hat{q} \in \hat{\mathcal{Q}} \backslash \Delta$ is a numbered configuration, for which we also write $\hat{q}=(\hat{x}, \hat{y})=\left(\boldsymbol{x}_{1}, \ldots, \boldsymbol{x}_{n}, \boldsymbol{y}_{1}, \ldots, \boldsymbol{y}_{m}\right)$. We also use the symmetrized and normalized kets $|s \hat{q}\rangle=s|\hat{q}\rangle$. As in (36), we can write

$$
P(d q)=\sum_{\hat{q} \in \pi^{-1}(q)}|\hat{q}\rangle\langle\hat{q}|d q=n ! m ! S| \hat{q}\rangle\langle\hat{q}|d q=| s \hat{q}\rangle\langle s \hat{q}| d q
$$

with arbitrary $\hat{q} \in \pi^{-1}(q)$. For the probability distribution, we thus have, as in (37),

$$
\mathbb{P}(d q)=\sum_{\hat{q} \in \pi^{-1}(q)}|\Psi(\hat{q})|^{2} d q=n ! m !|\Psi(\hat{q})|^{2} d q=|\langle s \hat{q} \mid \Psi\rangle|^{2} d q
$$

with arbitrary $\hat{q} \in \pi^{-1}(q)$.

The free Hamiltonian is the second quantized Schrödinger operator (with zero potential), associated with the free process described in Section 2.8, The interaction Hamiltonian is defined by

$$
H_{I}=\int d^{3} \boldsymbol{x} \psi^{\dagger}(\boldsymbol{x})\left(a_{\varphi}^{\dagger}(\boldsymbol{x})+a_{\varphi}(\boldsymbol{x})\right) \psi(\boldsymbol{x})
$$

with $\psi^{\dagger}(\boldsymbol{x})$ the creation operators (in position representation), acting on the fermion Fock space, and $a_{\varphi}^{\dagger}(\boldsymbol{x})$ the creation operators (in position representation), acting on the boson Fock space, regularized through convolution with an $L^{2}$ function $\varphi: \mathbb{R}^{3} \rightarrow \mathbb{R}$. $H_{I}$ has a kernel; we will now obtain a formula for it, see (51) below. The $|s \hat{q}\rangle$ are connected to the creation operators according to

$$
|s \hat{q}\rangle=\psi^{\dagger}\left(\boldsymbol{x}_{n}\right) \cdots \psi^{\dagger}\left(\boldsymbol{x}_{1}\right) a^{\dagger}\left(\boldsymbol{y}_{m}\right) \cdots a^{\dagger}\left(\boldsymbol{y}_{1}\right)|0\rangle,
$$

where $|0\rangle \in \mathscr{H}$ denotes the vacuum state. A relevant fact is that the creation and annihilation operators $\psi^{\dagger}, \psi, a^{\dagger}$ and $a$ possess kernels. Using the canonical (anti-)commutation 
relations for $\psi$ and $a$, one obtains from (46) the following formulas for the kernels of $\psi(\boldsymbol{r})$ and $a(\boldsymbol{r}), \boldsymbol{r} \in \mathbb{R}^{3}$ :

$$
\begin{aligned}
\left\langle s \hat{q}|\psi(\boldsymbol{r})| s \hat{q}^{\prime}\right\rangle & =\delta_{n, n^{\prime}-1} \delta_{m, m^{\prime}} \delta^{3 n^{\prime}}\left(x \cup \boldsymbol{r}-x^{\prime}\right)(-1)^{\varrho\left((\hat{x}, \boldsymbol{r}), \hat{x}^{\prime}\right)} \delta^{3 m}\left(y-y^{\prime}\right) \\
\left\langle s \hat{q}|a(\boldsymbol{r})| s \hat{q}^{\prime}\right\rangle & =\delta_{n, n^{\prime}} \delta_{m, m^{\prime}-1} \delta^{3 n}\left(x-x^{\prime}\right)(-1)^{\varrho\left(\hat{x}, \hat{x}^{\prime}\right)} \delta^{3 m^{\prime}}\left(y \cup \boldsymbol{r}-y^{\prime}\right)
\end{aligned}
$$

where $(x, y)=q=\pi(\hat{q})$, and $\varrho\left(\hat{x}, \hat{x}^{\prime}\right)$ denotes the permutation that carries $\hat{x}$ to $\hat{x}^{\prime}$ given that $x=x^{\prime}$. The corresponding formulas for $\psi^{\dagger}$ and $a^{\dagger}$ can be obtained by exchanging $\hat{q}$ and $\hat{q}^{\prime}$ on the right hand sides of (47) and (48). For the smeared-out operator $a_{\varphi}(\boldsymbol{r})$, we obtain

$$
\left\langle s \hat{q}\left|a_{\varphi}(\boldsymbol{r})\right| s \hat{q}^{\prime}\right\rangle=\delta_{n, n^{\prime}} \delta_{m, m^{\prime}-1} \delta^{3 n}\left(x-x^{\prime}\right)(-1)^{\varrho\left(\hat{x}, \hat{x}^{\prime}\right)} \sum_{\boldsymbol{y}^{\prime} \in y^{\prime}} \delta^{3 m}\left(y-y^{\prime} \backslash \boldsymbol{y}^{\prime}\right) \varphi\left(\boldsymbol{y}^{\prime}-\boldsymbol{r}\right)
$$

We make use of the resolution of the identity

$$
I=\int_{\mathcal{Q}} d q|s \hat{q}\rangle\langle s \hat{q}|
$$

Inserting (50) twice into (45) and exploiting (47) and (49), we find

$$
\begin{aligned}
\left\langle s \hat{q}\left|H_{I}\right| s \hat{q}^{\prime}\right\rangle & =\delta_{n, n^{\prime}} \delta_{m-1, m^{\prime}} \delta^{3 n}\left(x-x^{\prime}\right)(-1)^{\varrho\left(\hat{x}, \hat{x}^{\prime}\right)} \sum_{\boldsymbol{y} \in y} \delta^{3 m^{\prime}}\left(y \backslash \boldsymbol{y}-y^{\prime}\right) \sum_{\boldsymbol{x} \in x} \varphi(\boldsymbol{y}-\boldsymbol{x}) \\
& +\delta_{n, n^{\prime}} \delta_{m^{\prime}-1, m} \delta^{3 n}\left(x-x^{\prime}\right)(-1)^{\varrho\left(\hat{x}, \hat{x}^{\prime}\right)} \sum_{\boldsymbol{y}^{\prime} \in y^{\prime}} \delta^{3 m}\left(y-y^{\prime} \backslash \boldsymbol{y}^{\prime}\right) \sum_{\boldsymbol{x} \in x} \varphi\left(\boldsymbol{y}^{\prime}-\boldsymbol{x}\right)
\end{aligned}
$$

By (43), the jump rates (29) are

$$
\sigma\left(q \mid q^{\prime}\right)=\frac{\left[\frac{2}{\hbar} \operatorname{Im}\langle\Psi \mid s \hat{q}\rangle\left\langle s \hat{q}\left|H_{I}\right| s \hat{q}^{\prime}\right\rangle\left\langle s \hat{q}^{\prime} \mid \Psi\right\rangle\right]^{+}}{\left\langle\Psi \mid s \hat{q}^{\prime}\right\rangle\left\langle s \hat{q}^{\prime} \mid \Psi\right\rangle} .
$$

More explicitly, we obtain from (51) the rates

$$
\begin{aligned}
\sigma\left(q \mid q^{\prime}\right) & =\delta_{n n^{\prime}} \delta_{m-1, m^{\prime}} \delta^{3 n}\left(x-x^{\prime}\right) \sum_{\boldsymbol{y} \in y} \delta^{3 m^{\prime}}\left(y \backslash \boldsymbol{y}-y^{\prime}\right) \sigma_{\mathrm{crea}}\left(q^{\prime} \cup \boldsymbol{y} \mid q^{\prime}\right) \\
& +\delta_{n n^{\prime}} \delta_{m, m^{\prime}-1} \delta^{3 n}\left(x-x^{\prime}\right) \sum_{\boldsymbol{y}^{\prime} \in y^{\prime}} \delta^{3 m}\left(y-y^{\prime} \backslash \boldsymbol{y}^{\prime}\right) \sigma_{\mathrm{ann}}\left(q^{\prime} \backslash \boldsymbol{y}^{\prime} \mid q^{\prime}\right)
\end{aligned}
$$

with

$$
\begin{aligned}
\sigma_{\text {crea }}\left(q^{\prime} \cup \boldsymbol{y} \mid q^{\prime}\right) & =\frac{2 \sqrt{m^{\prime}+1}}{\hbar} \frac{\left[\operatorname{Im} \Psi^{*}(\hat{q})(-1)^{\varrho\left(\hat{x}, \hat{x}^{\prime}\right)} \sum_{\boldsymbol{x}^{\prime} \in x^{\prime}} \varphi\left(\boldsymbol{y}-\boldsymbol{x}^{\prime}\right) \Psi\left(\hat{q}^{\prime}\right)\right]^{+}}{\Psi^{*}\left(\hat{q}^{\prime}\right) \Psi\left(\hat{q}^{\prime}\right)} \\
\sigma_{\text {ann }}\left(q^{\prime} \backslash \boldsymbol{y}^{\prime} \mid q^{\prime}\right) & =\frac{2}{\hbar \sqrt{m^{\prime}}} \frac{\left[\operatorname{Im} \Psi^{*}(\hat{q})(-1)^{\varrho\left(\hat{x}, \hat{x}^{\prime}\right)} \sum_{\boldsymbol{x}^{\prime} \in x^{\prime}} \varphi\left(\boldsymbol{y}^{\prime}-\boldsymbol{x}^{\prime}\right) \Psi\left(\hat{q}^{\prime}\right)\right]^{+}}{\Psi^{*}\left(\hat{q}^{\prime}\right) \Psi\left(\hat{q}^{\prime}\right)},
\end{aligned}
$$


for arbitrary $\hat{q}^{\prime} \in \pi^{-1}\left(q^{\prime}\right)$ and $\hat{q} \in \pi^{-1}(q)$ with $q=\left(x^{\prime}, y^{\prime} \cup \boldsymbol{y}\right)$ respectively $q=\left(x^{\prime}, y^{\prime} \backslash \boldsymbol{y}^{\prime}\right)$. (Note that a sum sign can be drawn out of the plus function if the terms have disjoint supports.)

Equation (53) is worth looking at closely: One can read off that the only possible jumps are $\left(x^{\prime}, y^{\prime}\right) \rightarrow\left(x^{\prime}, y^{\prime} \cup \boldsymbol{y}\right)$, creation of a boson, and $\left(x^{\prime}, y^{\prime}\right) \rightarrow\left(x^{\prime}, y^{\prime} \backslash \boldsymbol{y}^{\prime}\right)$, annihilation of a boson. In particular, while one particle is created or annihilated, the other particles do not move. The process that we considered in [14] consists of pieces of Bohmian trajectories interrupted by jumps with rates (153); the process is thus an example of the jump rate formula (29), and an example of combining jumps and Bohmian motion by means of process additivity.

The example shows how, for other QFTs, the jump rates (29) can be applied to relevant interaction Hamiltonians: If $H_{I}$ is, in the position representation, a polynomial in the creation and annihilation operators, then it possesses a kernel on the relevant configuration space. A cut-off (implemented here by smearing out the creation and annihilation operators) needs to be introduced to make $H_{I}$ a well-defined operator on $L^{2}$.

If, in some QFT, the particle number operator is not conserved, jumps between the sectors of configuration space are inevitable for an equivariant process. And, indeed, when $H_{I}$ does not commute with the particle number operator (as is usually the case), jumps can occur that change the number of particles. Often, $H_{I}$ contains only offdiagonal terms with respect to the particle number; then every jump will change the particle number. This is precisely what happens in the model of [14].

\subsection{Efficient Calculation of Rates in the Previous Example}

We would like to give another, refined way of calculating the explicit jump rates (53) from the definition (45) of $H_{I}$. The calculation above is rather cumbersome, partly because of all the $\delta$ 's. It is also striking that only very few transitions $q^{\prime} \rightarrow q$ are actually possible, which suggests that it is unnecessary to write down a formula for the kernel $\left\langle q\left|H_{I}\right| q^{\prime}\right\rangle$ valid for all pairs $q, q^{\prime}$. Rather than writing down all the $\delta$ terms as in (53), it is easier to specify the possible transitions $q^{\prime} \rightarrow q$ and to write down the rates, such as (54a $)$ and (54b), only for these transitions. Thus, for a more efficient calculation of the rates, it is advisable to first determine the possible transitions, and then we need keep track only of the corresponding kernel elements.

\subsubsection{A Diagram Notation}

To formulate this more efficient strategy, it is helpful to regard $\Psi$ as a cross-section of a fiber bundle $E$ over the Riemannian manifold $\mathcal{Q}$, or of a countable union $E=\bigcup_{i} E^{(i)}$ of bundles $E^{(i)}$ over Riemannian manifolds $\mathcal{Q}^{(i)}$ with $\mathcal{Q}=\bigcup_{i} \mathcal{Q}^{(i)}$. (In the present example, with $\mathcal{Q}$ given by (40), we take $i$ to be the pair $(n, m)$ of particle numbers, $\mathcal{Q}^{(n, m)}$ to be the $(n, m)$-particle sector, and $E^{(i)}$ to be defined by (138) (with $\pi$ the natural projection from $\hat{\mathcal{Q}} \backslash \Delta$, with $\hat{\mathcal{Q}}$ given by (41), to $\mathcal{Q})$. The $\hat{q} \in \pi^{-1}(q)$ can be viewed as defining an orthonormal basis of $E_{q}$.) 
A key element of the strategy is a special diagram notation for operators. The operators we have in mind are $H_{I}$ and its building blocks, the field operators. The strategy will start with the diagrams for the field operators, and obtain from them a diagram for $H_{I}$. The diagram will specify, for an operator $O$, what the kernel of $O$ is, while leaving out parts of the kernel that are zero. So let us assume that $O$ has kernel $\left\langle q|O| q^{\prime}\right\rangle$, i.e., $(O \Psi)(q)=\int\left\langle q|O| q^{\prime}\right\rangle \Psi\left(q^{\prime}\right) d q^{\prime}$. The diagram

$$
q^{\prime} \underset{O}{\stackrel{K\left(q^{\prime}, \lambda\right)}{\longrightarrow}} F\left(q^{\prime}, \lambda\right)
$$

means that the operator $O$ has kernel constructed from $F$ and $K$,

$$
\left\langle q|O| q^{\prime}\right\rangle=\int_{\Lambda} d \lambda \delta\left(q-F\left(q^{\prime}, \lambda\right)\right) K\left(q^{\prime}, \lambda\right),
$$

where $\lambda$ varies in some parameter space $\Lambda, F: \mathcal{Q} \times \Lambda \rightarrow \mathcal{Q}$, and $K$ is a function (or distribution) of $q^{\prime}$ and $\lambda$ such that $K\left(q^{\prime}, \lambda\right): E_{q^{\prime}} \rightarrow E_{F\left(q^{\prime}, \lambda\right)}$ is a $\mathbb{C}$-linear mapping.

The role of $\lambda$ is to parametrize the possible transitions; e.g., for the boson creation (54a) in the previous section, $\lambda$ would be the position $\boldsymbol{y}$ of the new boson, and $\Lambda=\mathbb{R}^{3}$. The notation (55) does not explicitly mention what $\Lambda$ and the measure $d \lambda$ are; this will usually be clear from the context of the diagram. The measure $d \lambda$ will usually be a uniform distribution over the parameter space $\Lambda$, such as Lebesgue measure if $\Lambda=\mathbb{R}^{d}$ or the counting measure if $\Lambda$ is finite or countably infinite. We may also allow having a different $\Lambda_{q^{\prime}}$ for every $q^{\prime}$.

In words, (55) may be read as: "According to $O$, the possible transitions from $q$ ' are to $F\left(q^{\prime}, \lambda\right)$, and are associated with the amplitudes $K\left(q^{\prime}, \lambda\right)$." In fact, when $O=H$, a jump from $q^{\prime}$ can lead only to those $q$ 's for which $q=F\left(q^{\prime}, \lambda\right)$ for some value of $\lambda$, and the corresponding jump rate (29) is

$$
\sigma\left(F\left(q^{\prime}, \lambda\right) \mid q^{\prime}\right)=\frac{\left[(2 / \hbar) \operatorname{Im} \Psi^{*}\left(F\left(q^{\prime}, \lambda\right)\right) K\left(q^{\prime}, \lambda\right) \Psi\left(q^{\prime}\right)\right]^{+}}{\Psi^{*}\left(q^{\prime}\right) \Psi\left(q^{\prime}\right)}
$$

provided that for given $q^{\prime}, F\left(q^{\prime}, \cdot\right)$ is an injective mapping. Here, $\sigma\left(q \mid q^{\prime}\right)$ is the density of the measure $\sigma\left(d q \mid q^{\prime}\right)$ with respect to the measure on $\mathcal{Q}$

$$
\mu_{q^{\prime}}(d q)=\int_{\Lambda} d \lambda \delta\left(q-F\left(q^{\prime}, \lambda\right)\right) d q,
$$

where $\delta\left(q-q_{0}\right) d q$ denotes the measure on $\mathcal{Q}$ with total weight 1 concentrated at $q_{0}$. (58), the image of $d \lambda$ under the map $F\left(q^{\prime}, \cdot\right)$, is concentrated on the set $\left\{F\left(q^{\prime}, \lambda\right): \lambda \in \Lambda\right\}$ of possible destinations and plays the role of the "uniform distribution" over this set. In other words, (57) is the rate of occurrence, with respect to $d \lambda$, of the transition corresponding to $\lambda$. (For the boson creation rate (54a),$\mu_{q^{\prime}}(d q)$ turns out the Lebesgue measure in $\boldsymbol{y}$ on the subset $\left\{q^{\prime} \cup \boldsymbol{y}: \boldsymbol{y} \in \mathbb{R}^{3} \backslash q^{\prime}\right\} \subseteq \mathcal{Q}$.)

Given $O$, the choice of $\Lambda, F$, and $K$ is not unique. One could always choose $\Lambda=\mathcal{Q}$, $F\left(q^{\prime}, q\right)=q$, and $K\left(q^{\prime}, q\right)=\left\langle q|O| q^{\prime}\right\rangle$, which of course would mean to miss the point 
of this notation. The case that $F$ and $K$ do not depend on a parameter $\lambda$ is formally contained in the scheme (56) by taking $\Lambda$ to be a one-point set (and $d \lambda$ the counting measure); in this case (56) means

$$
\left\langle q|O| q^{\prime}\right\rangle=\delta\left(q-F\left(q^{\prime}\right)\right) K\left(q^{\prime}\right) .
$$

Conversely, whenever $\# \Lambda=1$, the dependence of $F$ and $K$ on the parameter $\lambda$ is irrelevant.

A basic advantage of the notation (55), compared to writing down a formula for $\left\langle q|O| q^{\prime}\right\rangle$, is that many $\delta$ factors become unnecessary. For example, if $O$ is multiplication by $V(q)$, then ( $\Lambda$ is a one-point set and) we have the diagram

$$
q^{\prime} \stackrel{V\left(q^{\prime}\right)}{\longrightarrow} q^{\prime}
$$

\subsubsection{Operations With Diagrams}

For the product $\mathrm{O}_{2} \mathrm{O}_{1}$ of two operators given by diagrams, we have the diagram

$$
q^{\prime} \stackrel{K_{2}\left(F_{1}\left(q^{\prime}, \lambda_{1}\right), \lambda_{2}\right) K_{1}\left(q^{\prime}, \lambda_{1}\right)}{O_{2} O_{1}} F_{2}\left(F_{1}\left(q^{\prime}, \lambda_{1}\right), \lambda_{2}\right)
$$

with parameter space $\Lambda_{1} \times \Lambda_{2}$, for which we also write

$$
q^{\prime} \stackrel{K_{1}\left(q^{\prime}, \lambda_{1}\right)}{O_{1}} F_{1}\left(q^{\prime}, \lambda_{1}\right) \stackrel{K_{2}\left(F_{1}\left(q^{\prime}, \lambda_{1}\right), \lambda_{2}\right)}{O_{2}} F_{2}\left(F_{1}\left(q^{\prime}, \lambda_{1}\right), \lambda_{2}\right)
$$

We thus define the concatenation of two diagrams by means of the composition of the transition mappings and the product of the amplitudes, i.e., using obvious notation,

$$
q_{1} \stackrel{\alpha}{\rightarrow} q_{2} \stackrel{\beta}{\rightarrow} q_{3} \quad \text { means } \quad q_{1} \stackrel{\alpha \beta}{\rightarrow} q_{3}
$$

Thus, multiplication of operators corresponds to concatenation of diagrams.

For the sum $O_{1}+O_{2}$ of two operators given by diagrams with the same parameter space $\Lambda_{1}=\Lambda_{2}=\Lambda$ and the same transition mapping $F_{1}\left(q^{\prime}, \lambda\right)=F_{2}\left(q^{\prime}, \lambda\right)=F\left(q^{\prime}, \lambda\right)$, we have the diagram

$$
q^{\prime} \underset{O_{1}\left(q^{\prime}, \lambda\right)+K_{2}\left(q^{\prime}, \lambda\right)}{O_{1}+O_{2}} F\left(q^{\prime}, \lambda\right)
$$

\subsubsection{Diagrams of Creation and Annihilation Operators}

We now write down diagrams for creation and annihilation operators. In the case that $O=O(\boldsymbol{r})$ arises from formally evaluating an operator-valued distribution $O(\boldsymbol{x})$ at $\boldsymbol{x}=\boldsymbol{r}$, the dependence of $K\left(q^{\prime}, \lambda\right)$ on $\lambda$ is in the sense of distributions rather than functions. More precisely, we have

$$
K\left(q^{\prime}, \lambda\right)=D\left(q^{\prime}, \lambda\right) K_{0}\left(q^{\prime}, \lambda\right)
$$

where $D$ is a (real-valued) distribution on $\mathcal{Q} \times \Lambda$, and $K_{0}$ a mapping-valued function such that for every $q^{\prime}$ and $\lambda, K_{0}\left(q^{\prime}, \lambda\right)$ is a linear mapping $E_{q^{\prime}} \rightarrow E_{F\left(q^{\prime}, \lambda\right)}$. 
For $\psi^{\dagger}(\boldsymbol{r})$ and $\psi(\boldsymbol{r}), \boldsymbol{r} \in \mathbb{R}^{3}$, we have (recall that $x^{\prime}$ is a finite subset of $\mathbb{R}^{3}$ )

$$
\begin{array}{ll}
\left(x^{\prime}, y^{\prime}\right) \underset{\psi^{\dagger}(\boldsymbol{r})}{\stackrel{\alpha_{\mathrm{f}}}{\longrightarrow}}\left(x^{\prime} \cup \boldsymbol{r}, y^{\prime}\right) & (\# \Lambda=1) \\
\left(x^{\prime}, y^{\prime}\right) \underset{\psi\left(\boldsymbol{x}^{\prime}-\boldsymbol{r}\right) \varepsilon_{\mathrm{f}}}{\stackrel{(\boldsymbol{r})}{\longrightarrow}}\left(x^{\prime} \backslash \boldsymbol{x}^{\prime}, y^{\prime}\right) & \left(\Lambda=x^{\prime}, \lambda=\boldsymbol{x}^{\prime}\right)
\end{array}
$$

using linear mappings $\alpha_{\mathrm{f}}: E_{q^{\prime}} \rightarrow E_{\left(x^{\prime} \cup \boldsymbol{r}, y^{\prime}\right)}$ ("append a fermion") and $\varepsilon_{\mathrm{f}}: E_{q^{\prime}} \rightarrow E_{\left(x^{\prime} \backslash \boldsymbol{x}^{\prime}, y^{\prime}\right)}$ ("erase a fermion"), which can be regarded as the natural mappings between these fiber spaces. They are defined through the following properties:

$\alpha_{\mathrm{f}} \Psi$ is appropriately symmetrized

$$
\begin{aligned}
& \left(\alpha_{\mathrm{f}} \Psi\right)\left(\left(\hat{x}^{\prime}, \boldsymbol{r}\right), \hat{y}^{\prime}\right)=\frac{1}{\sqrt{n^{\prime}+1}} \Psi\left(\hat{x}^{\prime}, \hat{y}^{\prime}\right) \\
& \left(\varepsilon_{\mathrm{f}} \Psi\right)\left(\hat{x}, \hat{y}^{\prime}\right)=\sqrt{n^{\prime}} \Psi\left(\left(\hat{x}, \boldsymbol{x}^{\prime}\right), \hat{y}^{\prime}\right)
\end{aligned}
$$

where $\Psi \in E_{q^{\prime}}$, and $\hat{x}$ is an arbitrary ordering of the set $x=x^{\prime} \backslash \boldsymbol{x}^{\prime}$. (Recall that the set $\pi^{-1}\left(q^{\prime}\right)$ of the possible orderings of $q^{\prime}$ forms a basis of $E_{q^{\prime}}$, so that every ordering $\left(\hat{x}^{\prime}, \hat{y}^{\prime}\right)=\hat{q}^{\prime} \in \pi^{-1}\left(q^{\prime}\right)$ corresponds to a particular component of $\Psi$. Thus, $\left(\left(\hat{x}^{\prime}, \boldsymbol{r}\right), \hat{y}^{\prime}\right) \in$ $\pi^{-1}\left(x^{\prime} \cup \boldsymbol{r}, y^{\prime}\right)$ corresponds to a particular component in $E_{\left(x^{\prime} \cup \boldsymbol{r}, y^{\prime}\right)}$.)

For the smeared-out creation and annihilation operators $a_{\varphi}^{\dagger}(\boldsymbol{r})$ and $a_{\varphi}(\boldsymbol{r})$, we have

$$
\begin{array}{ll}
\left(x^{\prime}, y^{\prime}\right) \underset{a_{\varphi}^{\dagger}(\boldsymbol{r})}{\stackrel{\varphi(\boldsymbol{y}-\boldsymbol{r}) \alpha_{\mathrm{b}}}{\longrightarrow}}\left(x^{\prime}, y^{\prime} \cup \boldsymbol{y}\right) & \left(\Lambda=\mathbb{R}^{3}, \lambda=\boldsymbol{y}\right) \\
\left(x^{\prime}, y^{\prime}\right) \underset{a_{\varphi}(\boldsymbol{r})}{\stackrel{\varphi\left(\boldsymbol{y}^{\prime}-\boldsymbol{r}\right) \varepsilon_{\mathrm{b}}}{\longrightarrow}}\left(x^{\prime}, y^{\prime} \backslash \boldsymbol{y}^{\prime}\right) & \left(\Lambda=y^{\prime}, \lambda=\boldsymbol{y}^{\prime}\right)
\end{array}
$$

where $\alpha_{\mathrm{b}}$ ("append a boson") and $\varepsilon_{\mathrm{b}}$ ("erase a boson") are the analogous linear mappings relating different spaces, $\alpha_{\mathrm{b}}: E_{q^{\prime}} \rightarrow E_{\left(x^{\prime}, y^{\prime} \cup \boldsymbol{y}\right)}$ and $\varepsilon_{\mathrm{b}}: E_{q^{\prime}} \rightarrow E_{\left(x^{\prime}, y^{\prime} \backslash \boldsymbol{y}^{\prime}\right)}$, defined by the following properties:

$$
\begin{aligned}
& \alpha_{\mathrm{b}} \Psi \text { is appropriately symmetrized } \\
& \left(\alpha_{\mathrm{b}} \Psi\right)\left(\hat{x}^{\prime},\left(\hat{y}^{\prime}, \boldsymbol{y}\right)\right)=\frac{1}{\sqrt{m^{\prime}+1}} \Psi\left(\hat{x}^{\prime}, \hat{y}^{\prime}\right) \\
& \left(\varepsilon_{\mathrm{b}} \Psi\right)\left(\hat{x}^{\prime}, \hat{y}\right)=\sqrt{m^{\prime}} \Psi\left(\hat{x}^{\prime},\left(\hat{y}, \boldsymbol{y}^{\prime}\right)\right),
\end{aligned}
$$

where $\hat{y}$ is an arbitrary ordering of the set $y=y^{\prime} \backslash \boldsymbol{y}^{\prime}, \hat{x}^{\prime}$ one of $x^{\prime}, \hat{y}^{\prime}$ one of $y^{\prime}$, and $\Psi \in E_{q^{\prime}}$.

\subsubsection{Application of the Diagram Method}

Now let us apply the strategy to the example (45) of the previous section. For $\psi^{\dagger}(\boldsymbol{r}) a_{\varphi}^{\dagger}(\boldsymbol{r}) \psi(\boldsymbol{r})$, we have the diagram

$$
q^{\prime} \underset{\psi(\boldsymbol{r})}{\stackrel{\delta\left(\boldsymbol{x}^{\prime}-\boldsymbol{r}\right) \varepsilon_{\mathrm{f}}}{\longrightarrow}}\left(x^{\prime} \backslash \boldsymbol{x}^{\prime}, y^{\prime}\right) \underset{a_{\varphi}^{\dagger}(\boldsymbol{r})}{\stackrel{\varphi(\boldsymbol{y}-\boldsymbol{r}) \alpha_{\mathrm{b}}}{\longrightarrow}}\left(x^{\prime} \backslash \boldsymbol{x}^{\prime}, y^{\prime} \cup \boldsymbol{y}\right) \underset{\psi^{\dagger}(\boldsymbol{r})}{\stackrel{\alpha_{\mathrm{f}}}{\longrightarrow}}\left(x^{\prime} \backslash \boldsymbol{x}^{\prime} \cup \boldsymbol{r}, y^{\prime} \cup \boldsymbol{y}\right)
$$


with $\Lambda=x^{\prime} \times \mathbb{R}^{3}$. Using the concatenation rule (62), we can write instead

$$
q^{\prime} \underset{\psi^{\dagger}(\boldsymbol{r}) a_{\varphi}^{\dagger}(\boldsymbol{r}) \psi(\boldsymbol{r})}{\stackrel{\left(\boldsymbol{x}^{\prime}-\boldsymbol{r}\right) \varphi(\boldsymbol{y}-\boldsymbol{r}) \alpha_{\mathrm{f}} \alpha_{\mathrm{b}} \varepsilon_{\mathrm{f}}}{\longrightarrow}}\left(x^{\prime} \backslash \boldsymbol{x}^{\prime} \cup \boldsymbol{r}, y^{\prime} \cup \boldsymbol{y}\right) .
$$

Integrating over $d \boldsymbol{r}$, we obtain, since $x^{\prime} \backslash \boldsymbol{x}^{\prime} \cup \boldsymbol{r}$ may be replaced by $x^{\prime}$, which is independent of $\boldsymbol{x}^{\prime}$,

$$
q^{\prime} \underset{x^{\prime} \in x^{\prime}}{\stackrel{\sum^{\prime} \boldsymbol{r}^{\dagger}(\boldsymbol{r}) a_{\varphi}^{\dagger}(\boldsymbol{r}) \psi(\boldsymbol{r})}{\longrightarrow}}\left(x^{\prime}, y^{\prime} \cup \boldsymbol{y}\right)
$$

with $\Lambda=\mathbb{R}^{3}$. We have now taken care of one of two terms in (45), involving $a^{\dagger}$ rather than $a$. From (69) we read off, without a big calculation, that this term corresponds to jumps $\left(x^{\prime}, y^{\prime}\right) \rightarrow\left(x^{\prime}, y^{\prime} \cup \boldsymbol{y}\right)$, or creation of a boson. The corresponding jump rate is given by (57), and reads here:

$$
\sigma\left(x^{\prime}, y^{\prime} \cup \boldsymbol{y} \mid q^{\prime}\right)=\frac{2}{\hbar} \frac{\left[\operatorname{Im} \Psi^{*}\left(x^{\prime}, y^{\prime} \cup \boldsymbol{y}\right) \sum_{\boldsymbol{x}^{\prime} \in x^{\prime}} \varphi\left(\boldsymbol{y}-\boldsymbol{x}^{\prime}\right) \alpha_{\mathrm{f}} \alpha_{\mathrm{b}} \varepsilon_{\mathrm{f}} \Psi\left(q^{\prime}\right)\right]^{+}}{\Psi^{*}\left(q^{\prime}\right) \Psi\left(q^{\prime}\right)} .
$$

This result agrees with (54a) ${ }^{9}$

We treat the term $\int d \boldsymbol{r} \psi^{\dagger}(\boldsymbol{r}) a_{\varphi}(\boldsymbol{r}) \psi(\boldsymbol{r})$ in the same way: We begin with the diagram

$$
q^{\prime} \underset{\psi(\boldsymbol{r})}{\stackrel{\delta\left(\boldsymbol{x}^{\prime}-\boldsymbol{r}\right) \varepsilon_{\mathrm{f}}}{\longrightarrow}}\left(x^{\prime} \backslash \boldsymbol{x}^{\prime}, y^{\prime}\right) \underset{a_{\varphi}(\boldsymbol{r})}{\stackrel{\varphi\left(\boldsymbol{y}^{\prime}-\boldsymbol{r}\right) \varepsilon_{\mathrm{b}}}{\longrightarrow}}\left(x^{\prime} \backslash \boldsymbol{x}^{\prime}, y^{\prime} \backslash \boldsymbol{y}^{\prime}\right) \underset{\psi^{\dagger}(\boldsymbol{r})}{\stackrel{\alpha_{\mathrm{f}}}{\longrightarrow}}\left(x^{\prime} \backslash \boldsymbol{x}^{\prime} \cup \boldsymbol{r}, y^{\prime} \backslash \boldsymbol{y}^{\prime}\right)
$$

with $\Lambda=x^{\prime} \times y^{\prime}$. Then we integrate over $d \boldsymbol{r}$ and obtain the associated jump rate

$$
\sigma\left(x^{\prime}, y^{\prime} \backslash \boldsymbol{y}^{\prime} \mid q^{\prime}\right)=\frac{2}{\hbar} \frac{\left[\operatorname{Im} \Psi^{*}\left(x^{\prime}, y^{\prime} \backslash \boldsymbol{y}^{\prime}\right) \sum_{\boldsymbol{x}^{\prime} \in x^{\prime}} \varphi\left(\boldsymbol{y}^{\prime}-\boldsymbol{x}^{\prime}\right) \alpha_{\mathrm{f}} \varepsilon_{\mathrm{b}} \varepsilon_{\mathrm{f}} \Psi\left(q^{\prime}\right)\right]^{+}}{\Psi^{*}\left(q^{\prime}\right) \Psi\left(q^{\prime}\right)},
$$

which agrees with (54b). Finally, $H_{I}$ (the sum of both contributions) corresponds according to (29) to jumps which, since the two contributions have no transitions $q^{\prime} \rightarrow q$ in common (or, in other words, since their kernels have disjoint supports in $\mathcal{Q} \times \mathcal{Q}$ ), are either $q^{\prime} \rightarrow\left(x^{\prime}, y^{\prime} \cup \boldsymbol{y}\right)$, with rate (70) , or $q^{\prime} \rightarrow\left(x^{\prime}, y^{\prime} \backslash \boldsymbol{y}^{\prime}\right)$, with rate (71).

\subsection{Pair Creation in an External Field}

As our second example, we present the Bell-type version of a reasonable and often used QFT of electrons and positrons, in which the electromagnetic field is a background field 31. The Bell-type version exhibits pair creation and annihilation (in the literal sense) and employs various notions we have introduced: process additivity, the configuration space $\Gamma_{\neq}\left(\mathbb{R}^{3}\right)$ of a variable number of identical particles, the free process, POVMs which are not PVMs, and stochastic jumps.

\footnotetext{
${ }^{9}$ Here is why: First, $\Psi^{*}\left(q^{\prime}\right) \Psi\left(q^{\prime}\right)=n^{\prime} ! m^{\prime} ! \Psi^{*}\left(\hat{q}^{\prime}\right) \Psi\left(\hat{q}^{\prime}\right)$ because the inner product in $E_{q^{\prime}}$ involves summation over all $\hat{q}^{\prime} \in \pi^{-1}\left(q^{\prime}\right)$. Similarly, the square bracket in the numerator of (70) involves the inner product of $E_{\left(x^{\prime}, y^{\prime} \cup y^{\prime}\right)}$, consisting of $n^{\prime} !\left(m^{\prime}+1\right)$ ! contributions. The numberings $\hat{q}$ and $\hat{q}^{\prime}$ in (54a) can be so chosen that $\hat{x}=\hat{x}^{\prime}, \boldsymbol{x}^{\prime}$ gets the last place of $\hat{x}^{\prime}$, and $\hat{y}=\hat{y}^{\prime} \cup \boldsymbol{y}^{\prime}$; then $\varrho\left(\hat{x}, \hat{x}^{\prime}\right)$ is trivial, and $\alpha_{\mathrm{f}} \alpha_{\mathrm{b}} \varepsilon_{\mathrm{f}} \Psi(\hat{q})=\left(n^{\prime}\right)^{-1 / 2}\left(m^{\prime}+1\right)^{-1 / 2}\left(n^{\prime}\right)^{1 / 2} \Psi\left(\hat{q}^{\prime}\right)$. Thus, the square bracket in (70) is $n^{\prime} ! m^{\prime} ! \sqrt{m^{\prime}+1}$ times the square bracket in (54a).
} 


\subsubsection{Fock Space and Hamiltonian}

We consider the second quantized Dirac field in an electromagnetic background field $A_{\mu}(\boldsymbol{x}, t)$. In terms of field operators, the Hamiltonian reads

$$
H=\int d^{3} x: \Phi^{*}(\boldsymbol{x})\left[-i c \hbar \boldsymbol{\alpha} \cdot \nabla+\beta m c^{2}+e\left(\boldsymbol{\alpha} \cdot \boldsymbol{A}+A_{0}\right)\right] \Phi(\boldsymbol{x}):,
$$

with colons denoting normal ordering. Note that $H$ is time-dependent due to the timedependence of $A_{\mu}(\boldsymbol{x}, t)$; more precisely, $H_{I}$ is time-dependent while $H_{0}$ is fixed. As a consequence, the relevant jump rate (29) is now time-dependent in three ways: through $H_{I}$, through $\Psi$, and through $q^{\prime}=Q_{t}$.

We quickly recall what the Hilbert space and the field operators are, and specify what POVM we use. After that, we construct the associated process.

The Hilbert space $L^{2}\left(\mathbb{R}^{3}, \mathbb{C}^{4}\right)$ of the Dirac equation is split into the orthogonal sum $\mathscr{H}_{+} \oplus \mathscr{H}_{-}$of the positive and negative energy subspaces of the free Dirac operator,

$$
h_{0}=-i c \hbar \boldsymbol{\alpha} \cdot \nabla+\beta m c^{2} .
$$

The 1-electron Hilbert space $\mathscr{H}_{\mathrm{e}}$ and the 1-positron Hilbert space $\mathscr{H}_{\mathrm{p}}$ are copies of $\mathscr{H}_{+}$, and the Fock space $\mathscr{F}=\Gamma \mathscr{H}^{(1)}$ arises then from the one-particle Hilbert space $\mathscr{H}^{(1)}=\mathscr{H}_{\mathrm{e}} \oplus \mathscr{H}_{\mathrm{p}}$ in the usual manner: with the anti-symmetrization operator Anti,

$$
\mathscr{F}=\bigoplus_{N=0}^{\infty} \operatorname{Anti}\left(\left(\mathscr{H}_{\mathrm{e}} \oplus \mathscr{H}_{\mathrm{p}}\right)^{\otimes N}\right)
$$

which can be naturally identified with

$$
\mathscr{H}:=\mathscr{F}_{\mathrm{e}} \otimes \mathscr{F}_{\mathrm{p}}=\bigoplus_{n=0}^{\infty} \operatorname{Anti}\left(\mathscr{H}_{\mathrm{e}}^{\otimes n}\right) \otimes \bigoplus_{\tilde{n}=0}^{\infty} \operatorname{Anti}\left(\mathscr{H}_{\mathrm{p}}^{\otimes \tilde{n}}\right) .
$$

Since $\mathscr{H}_{+} \subseteq L^{2}\left(\mathbb{R}^{3}, \mathbb{C}^{4}\right), \mathscr{H}$ can be understood as a subspace of

$$
\mathscr{H}_{\mathrm{ext}}:=\bigoplus_{n=0}^{\infty} \operatorname{Anti}\left(L^{2}\left(\mathbb{R}^{3}, \mathbb{C}^{4}\right)^{\otimes n}\right) \otimes \bigoplus_{\tilde{n}=0}^{\infty} \operatorname{Anti}\left(L^{2}\left(\mathbb{R}^{3}, \mathbb{C}^{4}\right)^{\otimes \tilde{n}}\right) .
$$

We choose the POVM and configuration space in the way suggested by the form (74), rather than (73):

$$
\mathcal{Q}=\Gamma_{\neq}\left(\mathbb{R}^{3}\right) \times \Gamma_{\neq}\left(\mathbb{R}^{3}\right),
$$

where the first factor represents electrons and the second positrons. (Recall from Section 2.8 that $\Gamma_{\neq}\left(\mathbb{R}^{3}\right)$ denotes the space of all finite subsets of $\mathbb{R}^{3}$. Another interesting possibility, suggested by the representation (73), is to set $\mathcal{Q}=\Gamma_{\neq}\left(\mathbb{R}^{3}\right)$. This would mean that, insofar as the configuration is concerned, electrons and positrons are not distinguished. However, we will not pursue this possibility here.) The natural POVM $P$ 
(see Section 4.2.3 and Section 2.11) can be expressed as an extension from rectangular sets (the existence of such an extension is proved in Section 4.4 of [15]):

$$
P\left(B_{\mathrm{e}} \times B_{\mathrm{p}}\right)=\Gamma P^{(1)}\left(B_{\mathrm{e}}\right) \otimes \Gamma P^{(1)}\left(B_{\mathrm{p}}\right)
$$

with $P^{(1)}$ the POVM on $\mathscr{H}_{+}$that we considered before, arising by projection from the natural PVM on $L^{2}\left(\mathbb{R}^{3}, \mathbb{C}^{4}\right)$. Alternatively, $P$ can be viewed as arising, by projection to $\mathscr{H}$, and from $\hat{\mathcal{Q}}=\bigcup_{n=0}^{\infty}\left(\mathbb{R}^{3}\right)^{n} \times \bigcup_{\tilde{n}=0}^{\infty}\left(\mathbb{R}^{3}\right)^{\tilde{n}}$ to $\mathcal{Q}$, of the natural PVM on $\hat{\mathcal{Q}}$ acting on $\mathscr{H}_{\text {ext }}$. Note that $P$ represents the usual $|\Psi|^{2}$ distribution in the sense that for a configuration $q$ with electrons at $\boldsymbol{x}_{1}, \ldots, \boldsymbol{x}_{n}$ and positrons at $\widetilde{\boldsymbol{x}}_{1}, \ldots, \widetilde{\boldsymbol{x}}_{\tilde{n}}$, we have

$$
\mathbb{P}(d q)=\langle\Psi|P(d q)| \Psi\rangle=n ! \widetilde{n} !\left|\Psi^{(n, \widetilde{n})}\left(\boldsymbol{x}_{1}, \ldots, \widetilde{\boldsymbol{x}}_{\tilde{n}}\right)\right|^{2} d \boldsymbol{x}_{1} \cdots d \widetilde{\boldsymbol{x}}_{\tilde{n}}
$$

where $\Psi^{(n, \widetilde{n})}$ is just the wave function $\left(\mathbb{R}^{3}\right)^{n+\widetilde{n}} \rightarrow\left(\mathbb{C}^{4}\right)^{\otimes(n+\widetilde{n})}$ we get when we decompose the state vector in the manner suggested by (75).$\Psi$ is normalized so that

$$
\sum_{n, \widetilde{n}=0}^{\infty} \int d \boldsymbol{x}_{1} \cdots d \widetilde{\boldsymbol{x}}_{\tilde{n}}\left|\Psi^{(n, \widetilde{n})}\left(\boldsymbol{x}_{1}, \ldots, \widetilde{\boldsymbol{x}}_{\tilde{n}}\right)\right|^{2}=1 .
$$

The field operator is defined by

$$
\Phi(f)=b\left(P_{+} f\right)+d^{*}\left(C P_{-} f\right)
$$

where $f$ is a test function from $L^{2}\left(\mathbb{R}^{3}, \mathbb{C}^{4}\right), P_{ \pm}$is the projection to $\mathscr{H}_{ \pm} \subseteq L^{2}\left(\mathbb{R}^{3}, \mathbb{C}^{4}\right), C$ is the charge conjugation operator which maps $\mathscr{H}_{-}$to $\mathscr{H}_{+}$and vice versa, and $b$ is the electron annihilation and $d^{*}$ the positron creation operator. Letting $\boldsymbol{e}_{i}$ be the standard orthonormal basis of $\mathbb{C}^{4}, i=1,2,3,4, \Phi(\boldsymbol{x})$ stands for $\Phi_{i}(\boldsymbol{x})=\Phi\left(\boldsymbol{e}_{i} \delta(\cdot-\boldsymbol{x})\right)$, where $i$ gets contracted with the $\boldsymbol{\alpha}$ matrices. Similarly, we define, as usual,

$$
\begin{aligned}
b_{i}(\boldsymbol{x}) & =b\left(P_{+}\left(\boldsymbol{e}_{i} \delta(\cdot-\boldsymbol{x})\right)\right) \\
\text { and } d_{i}(\boldsymbol{x}) & =d\left(C P_{-}\left(\boldsymbol{e}_{i} \delta(\cdot-\boldsymbol{x})\right)\right) .
\end{aligned}
$$

We thus have $\Phi_{i}(\boldsymbol{x})=b_{i}(\boldsymbol{x})+d_{i}^{*}(\boldsymbol{x})$.

\subsubsection{The Associated Process}

We now describe the associated Markov process. The free part of (72),

$$
H_{0}=\int d^{3} x: \Phi^{*}(\boldsymbol{x})\left[-i c \hbar \boldsymbol{\alpha} \cdot \nabla+\beta m c^{2}\right] \Phi(\boldsymbol{x}):
$$

preserves particle numbers (it commutes with the electron and positron number operators), evolving the $(n, \widetilde{n})$-particle sector of the Fock space according to the free $(n, \widetilde{n})$ particle Hamiltonian

$$
H_{0}^{(n, \widetilde{n})}=\sum_{k=1}^{n} h_{0}^{(k)}+\sum_{\widetilde{k}=1}^{\widetilde{n}} \widetilde{h}_{0}^{(\widetilde{k})}
$$


with

$$
\begin{aligned}
& h_{0}^{(k)}=-i c \hbar \boldsymbol{\alpha}^{(k)} \cdot \nabla_{k}+\beta^{(k)} m c^{2} \\
& \widetilde{h}_{0}^{(\widetilde{k})}=-i c \hbar \widetilde{\boldsymbol{\alpha}}^{(\widetilde{k})} \cdot \widetilde{\nabla}_{\widetilde{k}}+\widetilde{\beta}^{(\widetilde{k})} m c^{2}
\end{aligned}
$$

where $\boldsymbol{\alpha}^{(k)}$ and $\beta^{(k)}$ act on the $k$-th electron index in the tensor product representation (74) and $\widetilde{\boldsymbol{\alpha}}^{(\widetilde{k})}$ and $\widetilde{\beta}^{(\widetilde{k})}$ on the $\widetilde{k}$-th positron index. $\widetilde{\nabla}_{\widetilde{k}}$ is the gradient with respect to $\widetilde{\boldsymbol{x}}_{\tilde{k}}$.

With $H_{0}$ is associated a deterministic motion of the configuration in $\mathcal{Q}$, the free process introduced in Section 2.8. During this motion, the actual numbers $N, \widetilde{N}$ of electrons and positrons remain constant, while the positions $\left(\boldsymbol{X}_{1}, \ldots, \boldsymbol{X}_{N}, \widetilde{\boldsymbol{X}}_{1}, \ldots, \widetilde{\boldsymbol{X}}_{\widetilde{N}}\right)=: Q$ move according to Bohm-Dirac velocities (31), i.e.

$$
\begin{aligned}
& \dot{\boldsymbol{X}}_{k}=c \frac{\Psi^{*}(Q) \boldsymbol{\alpha}^{(k)} \Psi(Q)}{\Psi^{*}(Q) \Psi(Q)} \\
& \dot{\widetilde{\boldsymbol{X}}}_{\widetilde{k}}=c \frac{\Psi^{*}(Q) \widetilde{\boldsymbol{\alpha}}^{(\widetilde{k})} \Psi(Q)}{\Psi^{*}(Q) \Psi(Q)}
\end{aligned}
$$

where numerators and denominators are scalar products in $\left(\mathbb{C}^{4}\right)^{\otimes(N+\widetilde{N})}$.

We turn now to the interaction part. Setting $A=\boldsymbol{\alpha} \cdot e \boldsymbol{A}+e A_{0}$, we have that

$$
\begin{aligned}
H_{I}= & \int d^{3} \boldsymbol{x}: \Phi^{*}(\boldsymbol{x}) A(\boldsymbol{x}) \Phi(\boldsymbol{x}):= \\
= & \sum_{i, j=1}^{4} \int d^{3} \boldsymbol{x}:\left(b_{i}^{*}(\boldsymbol{x})+d_{i}(\boldsymbol{x})\right) A^{i, j}(\boldsymbol{x})\left(b_{j}(\boldsymbol{x})+d_{j}^{*}(\boldsymbol{x})\right):= \\
= & \sum_{i, j=1}^{4} \int d^{3} \boldsymbol{x}\left(b_{i}^{*}(\boldsymbol{x}) A^{i, j}(\boldsymbol{x}) b_{j}(\boldsymbol{x})+d_{i}(\boldsymbol{x}) A^{i, j}(\boldsymbol{x}) b_{j}(\boldsymbol{x})+\right. \\
& \left.+b_{i}^{*}(\boldsymbol{x}) A^{i, j}(\boldsymbol{x}) d_{j}^{*}(\boldsymbol{x})-d_{j}^{*}(\boldsymbol{x}) A^{i, j}(\boldsymbol{x}) d_{i}(\boldsymbol{x})\right) .
\end{aligned}
$$

Since $H_{I}$ is a polynomial in creation and annihilation operators, it possesses a kernel and corresponds to stochastic jumps. To compute the rates, we apply the strategy developed in Section 3.2. using diagrams. To this end, we regard fermionic wave functions again as cross-sections of a bundle $E$, defined here by

$$
E_{q}=\bigoplus_{\hat{q} \in \pi^{-1}(q)}\left(\mathbb{C}^{4}\right)^{\otimes n} \otimes\left(\mathbb{C}^{4}\right)^{\otimes \tilde{n}}
$$

Fermionic symmetry of a cross-section $\Psi$ of $E$ means that

$$
\begin{gathered}
\varrho\left(i_{1} \ldots i_{n}\right), \widetilde{\varrho}\left(\tilde{\imath}_{1} \ldots \tilde{\tau}_{\tilde{n}}\right) \\
\Psi\left(\varrho\left(\boldsymbol{x}_{1} \ldots \boldsymbol{x}_{n}\right), \varrho\left(\widetilde{\boldsymbol{x}}_{1} \ldots \widetilde{\boldsymbol{x}}_{\tilde{n}}\right)\right)=(-1)^{\varrho}(-1)^{\widetilde{\varrho}} \Psi\left(\boldsymbol{x}_{1} \ldots \boldsymbol{x}_{n}, \boldsymbol{x}_{1}, \tilde{\boldsymbol{x}}_{1} \ldots \tilde{i}_{\tilde{n}}\right. \\
\left.\widetilde{\boldsymbol{x}}_{\widetilde{n}}\right)
\end{gathered}
$$


for all permutations $\varrho \in S_{n}$ and $\widetilde{\varrho} \in S_{\widetilde{n}}$.

The diagrams for $b_{i}^{*}(\boldsymbol{x}), b_{i}(\boldsymbol{x}), d_{i}^{*}(\boldsymbol{x})$, and $d_{i}(\boldsymbol{x})$ are

$$
\begin{aligned}
& \left(x^{\prime}, \widetilde{x}^{\prime}\right) \stackrel{\sum_{j} S_{+_{i}^{j}}\left(\boldsymbol{x}^{\prime}-\boldsymbol{x}\right) \alpha_{\mathrm{e}}\left(\boldsymbol{e}_{j}\right)}{b_{i}^{*}(\boldsymbol{x})}\left(x^{\prime} \cup \boldsymbol{x}^{\prime}, \widetilde{x}^{\prime}\right) \\
& \left(x^{\prime}, \widetilde{x}^{\prime}\right) \stackrel{\sum_{j} S_{+}^{j}\left(\boldsymbol{x}^{\prime}-\boldsymbol{x}\right) \varepsilon_{\mathrm{e}}\left(\boldsymbol{e}_{j}\right)}{b_{i}(\boldsymbol{x})}\left(x^{\prime} \backslash \boldsymbol{x}^{\prime}, \widetilde{x}^{\prime}\right) \\
& \left(x^{\prime}, \widetilde{x}^{\prime}\right) \stackrel{\sum_{j} S_{-i}^{j}\left(\widetilde{\boldsymbol{x}}^{\prime}-\boldsymbol{x}\right) \alpha_{\mathrm{p}}\left(\boldsymbol{e}_{j}\right)}{d_{i}^{*}(\boldsymbol{x})}\left(x^{\prime}, \widetilde{x}^{\prime} \cup \widetilde{\boldsymbol{x}}^{\prime}\right) \\
& \left(x^{\prime}, \widetilde{x}^{\prime}\right) \stackrel{\sum_{j} S_{-i}^{j}\left(\widetilde{\boldsymbol{x}}^{\prime}-\boldsymbol{x}\right) \varepsilon_{\mathrm{p}}\left(\boldsymbol{e}_{j}\right)}{d_{i}(\boldsymbol{x})}\left(x^{\prime}, \widetilde{x}^{\prime} \backslash \widetilde{\boldsymbol{x}}^{\prime}\right)
\end{aligned}
$$

where the matrix function $S_{+j}^{i}(\boldsymbol{x})$ is defined as the $j$-component of $P_{+}\left(\boldsymbol{e}_{i} \delta(\cdot)\right)$, and $S_{-j}^{i}(\boldsymbol{x})$ as the $j$-component of $C P_{-}\left(\boldsymbol{e}_{i} \delta(\cdot)\right)$. The linear mappings $\alpha_{\mathrm{e}}\left(\boldsymbol{e}_{j}\right): E_{q^{\prime}} \rightarrow$ $E_{\left(x^{\prime} \cup \boldsymbol{x}^{\prime}, \tilde{x}^{\prime}\right)}$ ("append an electron with spinor $\boldsymbol{e}_{j}$ ") and $\varepsilon_{\mathrm{e}}\left(\boldsymbol{e}_{j}\right): E_{q^{\prime}} \rightarrow E_{\left(x^{\prime} \backslash \boldsymbol{x}^{\prime}, \tilde{x}^{\prime}\right)}$ ("erase an electron, contracting with spinor $\boldsymbol{e}_{j}$ ") are defined through their properties that for $\Psi \in E_{q^{\prime}}$,

$$
\begin{aligned}
& \alpha_{\mathrm{e}} \Psi \text { is appropriately symmetrized } \\
& \left(\alpha_{\mathrm{e}}\left(\boldsymbol{e}_{j}\right) \Psi\right)\left(\left(\hat{x}^{\prime}, \boldsymbol{x}^{\prime}\right), \hat{\widetilde{x}}^{\prime}\right)=\frac{1}{\sqrt{n^{\prime}+1}} \Psi\left(\hat{x}^{\prime}, \hat{\widehat{x}}^{\prime}\right) \otimes \boldsymbol{e}_{j} \\
& \left(\varepsilon_{\mathrm{e}}\left(\boldsymbol{e}_{j}\right) \Psi\right)\left(\hat{x}, \hat{\widetilde{x}}^{\prime}\right)=\sqrt{n^{\prime}} \Psi_{j}\left(\left(\hat{x}, \boldsymbol{x}^{\prime}\right), \hat{\vec{x}}^{\prime}\right),
\end{aligned}
$$

where $\hat{x}$ is an arbitrary ordering of $x=x^{\prime} \backslash \boldsymbol{x}^{\prime}, \hat{x}^{\prime}$ one of $x^{\prime}$, and $\hat{\vec{x}}^{\prime}$ one of $\widetilde{x}^{\prime}$. We refer to the last electron slot when writing the tensor product or taking the $j$-component. $\alpha_{\mathrm{p}}\left(\boldsymbol{e}_{j}\right)$ and $\varepsilon_{\mathrm{p}}\left(\boldsymbol{e}_{j}\right)$ are defined analogously.

For the four terms in (80c), we thus get the four diagrams (omitting the multiplication by $\left.A^{i, j}(\boldsymbol{x})\right)$

$$
\begin{aligned}
& \left(x^{\prime}, \widetilde{x}^{\prime}\right) \stackrel{\sum_{k} S_{+}^{k}\left(\boldsymbol{x}^{\prime}-\boldsymbol{x}\right) \varepsilon_{\mathrm{e}}\left(\boldsymbol{e}_{k}\right)}{b_{j}(\boldsymbol{x})}\left(x^{\prime} \backslash \boldsymbol{x}^{\prime}, \widetilde{x}^{\prime}\right) \frac{\sum_{\ell} S_{+}^{\ell}\left(\boldsymbol{x}^{\prime \prime}-\boldsymbol{x}\right) \alpha_{\mathrm{e}}\left(\boldsymbol{e}_{\ell}\right)}{b_{i}^{*}(\boldsymbol{x})}\left(x^{\prime} \backslash \boldsymbol{x}^{\prime} \cup \boldsymbol{x}^{\prime \prime}, \widetilde{x}^{\prime}\right) \\
& \left(x^{\prime}, \widetilde{x}^{\prime}\right) \stackrel{\sum_{k} S_{+}^{k}\left(\boldsymbol{x}^{\prime}-\boldsymbol{x}\right) \varepsilon_{\mathrm{e}}\left(\boldsymbol{e}_{k}\right)}{b_{j}(\boldsymbol{x})}\left(x^{\prime} \backslash \boldsymbol{x}^{\prime}, \widetilde{x}^{\prime}\right) \frac{\sum_{\ell} S_{-i}^{\ell}\left(\widetilde{\boldsymbol{x}}^{\prime}-\boldsymbol{x}\right) \varepsilon_{\mathrm{p}}\left(\boldsymbol{e}_{\ell}\right)}{d_{i}(\boldsymbol{x})}\left(x^{\prime} \backslash \boldsymbol{x}^{\prime}, \widetilde{x}^{\prime} \backslash \widetilde{\boldsymbol{x}}^{\prime}\right) \\
& \left(x^{\prime}, \widetilde{x}^{\prime}\right) \stackrel{\sum_{k} S_{-}^{k}\left(\widetilde{\boldsymbol{x}}^{\prime}-\boldsymbol{x}\right) \alpha_{\mathrm{p}}\left(\boldsymbol{e}_{k}\right)}{d_{j}^{*}(\boldsymbol{x})}\left(x^{\prime}, \widetilde{x}^{\prime} \cup \widetilde{\boldsymbol{x}}^{\prime}\right) \frac{\sum_{\ell} S_{+}^{\ell}\left(\boldsymbol{x}^{\prime}-\boldsymbol{x}\right) \alpha_{\mathrm{e}}\left(\boldsymbol{e}_{\ell}\right)}{b_{i}^{*}(\boldsymbol{x})}\left(x^{\prime} \cup \boldsymbol{x}^{\prime}, \widetilde{x}^{\prime} \cup \widetilde{\boldsymbol{x}}^{\prime}\right) \\
& \left(x^{\prime}, \widetilde{x}^{\prime}\right) \stackrel{\left.\sum_{k} S_{-}^{k}{ }_{i}^{\prime} \widetilde{\boldsymbol{x}}^{\prime}-\boldsymbol{x}\right) \varepsilon_{\mathrm{p}}\left(\boldsymbol{e}_{k}\right)}{d_{i}(\boldsymbol{x})}\left(x^{\prime}, \widetilde{x}^{\prime} \backslash \widetilde{\boldsymbol{x}}^{\prime}\right) \frac{\sum_{\ell} S_{-j}^{\ell}\left(\widetilde{\boldsymbol{x}}^{\prime \prime}-\boldsymbol{x}\right) \alpha_{\mathrm{p}}\left(\boldsymbol{e}_{\ell}\right)}{d_{j}^{*}(\boldsymbol{x})}\left(x^{\prime}, \widetilde{x}^{\prime} \backslash \widetilde{\boldsymbol{x}}^{\prime} \cup \widetilde{\boldsymbol{x}}^{\prime \prime}\right) .
\end{aligned}
$$

We read off that the first term corresponds to the jump of a single electron from $\boldsymbol{x}^{\prime}$ to $\boldsymbol{x}^{\prime \prime}$, while all other particles remain where they were, the second to the annihilation of an electron-positron pair at locations $\boldsymbol{x}^{\prime}$ and $\widetilde{\boldsymbol{x}}^{\prime}$, the third to the creation of an electronpositron pair at locations $\boldsymbol{x}^{\prime}$ and $\widetilde{\boldsymbol{x}}^{\prime}$, and the last to the jump of a positron from $\widetilde{\boldsymbol{x}}^{\prime}$ to 
$\widetilde{\boldsymbol{x}}^{\prime \prime}$. The corresponding jump rates are

$$
\begin{aligned}
\sigma_{\mathrm{e}}\left(x^{\prime} \backslash \boldsymbol{x}^{\prime} \cup \boldsymbol{x}^{\prime \prime}, \widetilde{x}^{\prime} \mid q^{\prime}\right) & =\frac{\left[(2 / \hbar) \operatorname{Im} \Psi^{*}(q) \sum_{k, \ell} \chi_{\mathrm{e}}^{k, \ell}\left(\boldsymbol{x}^{\prime}, \boldsymbol{x}^{\prime \prime}\right) \alpha_{\mathrm{e}}\left(\boldsymbol{e}_{\ell}\right) \varepsilon_{\mathrm{e}}\left(\boldsymbol{e}_{k}\right) \Psi\left(q^{\prime}\right)\right]^{+}}{\Psi^{*}\left(q^{\prime}\right) \Psi\left(q^{\prime}\right)} \\
\sigma_{\mathrm{ann}}\left(x^{\prime} \backslash \boldsymbol{x}^{\prime}, \widetilde{x}^{\prime} \backslash \widetilde{\boldsymbol{x}}^{\prime} \mid q^{\prime}\right) & =\frac{\left[(2 / \hbar) \operatorname{Im} \Psi^{*}(q) \sum_{k, \ell} \chi_{\mathrm{ann}}^{k, \ell}\left(\boldsymbol{x}^{\prime}, \widetilde{\boldsymbol{x}}^{\prime}\right) \varepsilon_{\mathrm{p}}\left(\boldsymbol{e}_{\ell}\right) \varepsilon_{\mathrm{e}}\left(\boldsymbol{e}_{k}\right) \Psi\left(q^{\prime}\right)\right]^{+}}{\Psi^{*}\left(q^{\prime}\right) \Psi\left(q^{\prime}\right)} \\
\sigma_{\text {crea }}\left(x^{\prime} \cup \boldsymbol{x}^{\prime}, \widetilde{x}^{\prime} \cup \widetilde{\boldsymbol{x}}^{\prime} \mid q^{\prime}\right) & =\frac{\left[(2 / \hbar) \operatorname{Im} \Psi^{*}(q) \sum_{k, \ell} \chi_{\mathrm{crea}}^{k, \ell}\left(\boldsymbol{x}^{\prime}, \widetilde{\boldsymbol{x}}^{\prime}\right) \alpha_{\mathrm{e}}\left(\boldsymbol{e}_{\ell}\right) \alpha_{\mathrm{p}}\left(\boldsymbol{e}_{k}\right) \Psi\left(q^{\prime}\right)\right]^{+}}{\Psi^{*}\left(q^{\prime}\right) \Psi\left(q^{\prime}\right)} \\
\sigma_{\mathrm{p}}\left(x^{\prime}, \widetilde{x}^{\prime} \backslash \widetilde{\boldsymbol{x}}^{\prime} \cup \widetilde{\boldsymbol{x}}^{\prime \prime} \mid q^{\prime}\right) & =\frac{\left[(2 / \hbar) \operatorname{Im} \Psi^{*}(q) \sum_{k, \ell} \chi_{\mathrm{p}}^{k, \ell}\left(\widetilde{\boldsymbol{x}}^{\prime}, \widetilde{\boldsymbol{x}}^{\prime \prime}\right) \alpha_{\mathrm{p}}\left(\boldsymbol{e}_{\ell}\right) \varepsilon_{\mathrm{p}}\left(\boldsymbol{e}_{k}\right) \Psi\left(q^{\prime}\right)\right]^{+}}{\Psi^{*}\left(q^{\prime}\right) \Psi\left(q^{\prime}\right)},
\end{aligned}
$$

where $q$ denotes the respective destination, and

$$
\begin{aligned}
& \chi_{\mathrm{e}}^{k, \ell}\left(\boldsymbol{x}^{\prime}, \boldsymbol{x}^{\prime \prime}\right)=\sum_{i, j} \int d^{3} \boldsymbol{x} S_{+i}^{\ell}\left(\boldsymbol{x}^{\prime \prime}-\boldsymbol{x}\right) A^{i, j}(\boldsymbol{x}) S_{+j}^{k}\left(\boldsymbol{x}^{\prime}-\boldsymbol{x}\right) \\
& \chi_{\mathrm{ann}}^{k, \ell}\left(\boldsymbol{x}^{\prime}, \widetilde{\boldsymbol{x}}^{\prime}\right)=\sum_{i, j} \int d^{3} \boldsymbol{x} S_{-i}^{\ell}\left(\widetilde{\boldsymbol{x}}^{\prime}-\boldsymbol{x}\right) A^{i, j}(\boldsymbol{x}) S_{+j}^{k}\left(\boldsymbol{x}^{\prime}-\boldsymbol{x}\right) \\
& \chi_{\text {crea }}^{k, \ell}\left(\boldsymbol{x}^{\prime}, \widetilde{\boldsymbol{x}}^{\prime}\right)=\sum_{i, j} \int d^{3} \boldsymbol{x} S_{+i}^{\ell}\left(\boldsymbol{x}^{\prime}-\boldsymbol{x}\right) A^{i, j}(\boldsymbol{x}) S_{-j}^{k}\left(\widetilde{\boldsymbol{x}}^{\prime}-\boldsymbol{x}\right) \\
& \chi_{\mathrm{p}}^{k, \ell}\left(\widetilde{\boldsymbol{x}}^{\prime}, \widetilde{\boldsymbol{x}}^{\prime \prime}\right)=-\sum_{i, j} \int d^{3} \boldsymbol{x} S_{-j}^{\ell}\left(\widetilde{\boldsymbol{x}}^{\prime \prime}-\boldsymbol{x}\right) A^{i, j}(\boldsymbol{x}) S_{-i}^{k}\left(\widetilde{\boldsymbol{x}}^{\prime}-\boldsymbol{x}\right) .
\end{aligned}
$$

The process for $H_{0}+H_{I}$ that we obtain through process additivity is the motion (79) interrupted by stochastic jumps with rates (86).

Note that the jump of a single electron has small probability to be across a distance much larger than the width of the functions $S_{ \pm}$, which is of the order of the Compton wavelength of the electron. Similarly, the distance $|\boldsymbol{x}-\widetilde{\boldsymbol{x}}|$ of a newly created pair, or of a pair at the moment of annihilation, has small probability to be much larger than the width of $S_{ \pm}$. While the jump of a single electron or positron leaves the number $N$ of electrons and the number $\widetilde{N}$ of positrons unchanged, pair creation and annihilation can only either decrease or increase both $N$ and $\widetilde{N}$ by 1 . As a consequence, the actual net charge $\widetilde{N}-N$ is conserved by the process.

\section{Second Quantization of a Markov Process}

\subsection{Preliminaries Concerning the Conditional Density Matrix}

In the next section, we describe the algorithm for the "second quantization" of a process. But before that, we have to introduce, as a preparation, the notion of a conditional density matrix. In [19], we have defined for Bohmian mechanics the conditional wave function of, say, subsystem 1 of a composite system with configuration space $\mathcal{Q}=$ 
$\mathcal{Q}_{1} \times \mathcal{Q}_{2}$ by $\Psi_{\text {cond }}\left(q_{1}\right)=\Psi\left(q_{1}, Q_{2}\right)$. From a complex wave function $\Psi: \mathcal{Q} \rightarrow \mathbb{C}$, together with the actual configuration $Q_{2}$ of the environment of the subsystem in the composite, we thus form a wave function $\Psi_{\text {cond }}: \mathcal{Q}_{1} \rightarrow \mathbb{C}$; for Bohmian mechanics with spin, in contrast, we would not, in general, obtain a suitable wave function for subsystems in this way, because $\Psi_{\text {cond }}$ as just defined would have more spin indices than appropriate. We can however still define the conditional density matrix for subsystem 1,

$$
W_{\text {cond } s_{1} s_{1}^{\prime}}\left(q_{1}, q_{1}^{\prime}\right)=\frac{1}{\gamma} \sum_{s_{2}} \Psi_{s_{1}, s_{2}}\left(q_{1}, Q_{2}\right) \Psi_{s_{1}^{\prime}, s_{2}}^{*}\left(q_{1}^{\prime}, Q_{2}\right)
$$

where the $s$ 's are spin indices. In order that $W$, like any density matrix, have trace 1 , the normalizing factor $\gamma$ must be chosen as

$$
\gamma=\int_{q_{1} \in \mathcal{Q}_{1}} \sum_{s_{1}, s_{2}} \Psi_{s_{1}, s_{2}}^{*}\left(q_{1}, Q_{2}\right) \Psi_{s_{1}, s_{2}}\left(q_{1}, Q_{2}\right) d q_{1} .
$$

This $W$ can play most of the roles of the conditional wave function in spinless Bohmian mechanics. The notion of a conditional density matrix easily generalizes from the situation just described, corresponding to wave functions in $L^{2}\left(\mathcal{Q}, \mathbb{C}^{k}\right)$ and the natural localization PVM, to the situation of any product localization POVM on any tensor product Hilbert space: for $\mathscr{H}=\mathscr{H}_{1} \otimes \mathscr{H}_{2}$ and $P\left(d q_{1} \times d q_{2}\right)=P_{1}\left(d q_{1}\right) \otimes P_{2}\left(d q_{2}\right)$, set

$$
W_{\text {cond }}=\left.\frac{\operatorname{tr}_{2}\left(|\Psi\rangle\langle\Psi| P\left(\mathcal{Q}_{1} \times d q_{2}\right)\right)}{\operatorname{tr}\left(|\Psi\rangle\langle\Psi| P\left(\mathcal{Q}_{1} \times d q_{2}\right)\right)}\right|_{q_{2}=Q_{2}},
$$

where $\operatorname{tr}_{2}$ is the partial trace over $\mathscr{H}_{2}$. The quotient is to be understood as a RadonNikodým derivative in $q_{2}$. Like conditional wave functions, conditional density matrices cannot be defined in orthodox quantum theory, for lack of the configuration $Q_{2}$. We stress that conditional density matrices have nothing, absolutely nothing, to do with statistical ensembles of state vectors in $\mathscr{H}_{1}$. Like any density matrix, they do, however, define a probability distribution on $\mathcal{Q}_{1}$,

$$
\mathbb{P}_{1}^{W_{\text {cond }}}(\cdot)=\operatorname{tr}\left(W_{\text {cond }} P_{1}(\cdot)\right),
$$

which coincides with the conditional distribution of $Q_{1}$ given $Q_{2}$,

$$
\mathbb{P}\left(Q_{1} \in \cdot \mid Q_{2}\right)=\left.\frac{\left\langle\Psi\left|P_{1}(\cdot) \otimes P_{2}\left(d q_{2}\right)\right| \Psi\right\rangle}{\left\langle\Psi\left|\mathbf{1} \otimes P_{2}\left(d q_{2}\right)\right| \Psi\right\rangle}\right|_{q_{2}=Q_{2}} .
$$

The evolution of $W_{\text {cond }}$ is not autonomous; it will typically depend on (and always be determined by) $\Psi_{t}$ and $Q_{2, t}$. For a given density matrix $W$ of a system that is not regarded as a subsystem, however, one can define (as usual) the time evolution by $W_{t}=e^{-i H t / \hbar} W e^{i H t / \hbar}$, which gives rise to a time-dependent distribution $\mathbb{P}^{W_{t}}(\cdot)=$ $\operatorname{tr}\left(W_{t} P(\cdot)\right)$. We call a Markov process that is $\mathbb{P}^{W_{t}}$-distributed at every time $t$ equivariant 
with respect to $W$ and $H$. Given the right initial distribution, this is equivalent to the following condition on the generator:

$$
\mathscr{L} \mathbb{P}^{W}(\cdot)=\frac{2}{\hbar} \operatorname{Im} \operatorname{tr}(W P(\cdot) H) .
$$

This is the version of (12) for density matrices, and defines an equivariant generator with respect to $W$ and $H$.

Since conditional density matrices will play a crucial role in the construction of the many-particle process, we require that, as part of the input data of the algorithm, we are given an equivariant generator $\mathscr{L}_{W}^{(1)}$ for every density matrix from a dense subset of the density matrices in $\mathscr{H}^{(1) *} \otimes \mathscr{H}^{(1)}$. This is not much of a restriction, as all relevant examples of equivariant generators naturally extend to density matrices: Bohmian mechanics with spin space $\mathbb{C}^{k}$ can be extended [4] to

$$
v^{W}(q)=\hbar \operatorname{Im} \frac{\nabla_{q} \operatorname{tr}_{\mathbb{C}^{k}} W\left(q, q^{\prime}\right)}{\operatorname{tr}_{\mathbb{C}^{k}} W\left(q, q^{\prime}\right)}\left(q^{\prime}=q\right),
$$

Bohm-Dirac to

$$
v^{W}(q)=\frac{\operatorname{tr}_{\mathbb{C}^{4}}(W(q, q) \boldsymbol{\alpha})}{\operatorname{tr}_{\mathbb{C}^{4}}(W(q, q))}
$$

and minimal jump rates to

$$
\sigma^{W}\left(d q \mid q^{\prime}\right)=\frac{\left[(2 / \hbar) \operatorname{Im} \operatorname{tr}\left(W P(d q) H P\left(d q^{\prime}\right)\right)\right]^{+}}{\operatorname{tr}\left(W P\left(d q^{\prime}\right)\right)} .
$$

Note also that (92) would not make any sense if $W$ represented a statistical ensemble [4, whereas it makes good sense for conditional density matrices, expressing the true relation between the Bohmian velocity for a subsystem arising from (11) and the conditional density matrix (88) of that subsystem. Mutatis mutandis, the same is true of (93). Similarly, in case that $P$ is a PVM, (94) expresses the jump rates for a decoupled subsystem arising from (29) for the composite in terms of the conditional density matrix of that subsystem.

\subsection{Algorithm}

The input data of this algorithm are the one-particle Hilbert space $\mathscr{H}^{(1)}$, configuration space $\mathcal{Q}^{(1)}$, POVM $P^{(1)}$, and a family of generators $\mathscr{L}^{(1)}=\mathscr{L}_{W}^{(1)}$ labeled by the density matrices $W$ from a dense subset of the density matrices in $\mathscr{H}^{(1) *} \otimes \mathscr{H}^{(1)}$. The output is a family of generators $\Gamma \mathscr{L}^{(1)}=\mathscr{L}_{0}=\mathscr{L}_{0, \Psi}$ labeled by the state vectors $\Psi$ in (a dense subspace of) Fock space. If $\mathscr{L}_{W}^{(1)}$ is equivariant with respect to $W$ and $H^{(1)}$, then $\mathscr{L}_{0, \Psi}$ is equivariant with respect to $\Psi$ and $H_{0}$.

The algorithm is based on two procedures for suitably combining generators for direct sums or tensor products of Hilbert spaces. 


\subsubsection{Direct Sums}

Given a finite or countable sequence of Hilbert spaces $\mathscr{H}^{(n)}$ with POVMs $P^{(n)}$ on configuration spaces $\mathcal{Q}^{(n)}$, and for each $n$ a family of generators $\mathscr{L}^{(n)}$ labeled by the vectors in $\mathscr{H}^{(n)}$, there is a canonically constructed family of generators $\mathscr{L}^{\oplus}=\mathscr{L}_{\Psi}^{\oplus}$, labeled by the vectors in the direct sum $\bigoplus_{n} \mathscr{H}^{(n)}$. The space $\mathcal{Q}$ in which the corresponding process takes place is the disjoint union of the $\mathcal{Q}^{(n)}$. If every $\mathscr{L}_{\Psi_{n}}^{(n)}$ is equivariant with respect to $\Psi_{n} \in \mathscr{H}^{(n)}$ and $H^{(n)}$, then $\mathscr{L}_{\Psi}^{\oplus}$ is equivariant with respect to $\Psi \in \bigoplus_{n} \mathscr{H}^{(n)}$ and $\bigoplus_{n} H^{(n)}$.

Here are the details. The POVM $P=\bigoplus_{n} P^{(n)}$ on $\mathcal{Q}$ that naturally arises from the data is given by $P(B)=\bigoplus_{n} P^{(n)}\left(B \cap \mathcal{Q}^{(n)}\right)$ for $B \subseteq \mathcal{Q}$. Let $P_{n}$ denote the projection $\mathscr{H} \rightarrow \mathscr{H}^{(n)}$. The generator $\mathscr{L}^{\oplus}$ is given by

$$
\left.\left(\mathscr{L}_{\Psi}^{\oplus} \rho\right)\right|_{\mathcal{Q}^{(n)}}=\mathscr{L}_{P_{n} \Psi /\left\|P_{n} \Psi\right\|}^{(n)}\left(\left.\rho\right|_{\mathcal{Q}^{(n)}}\right) .
$$

It generates a (Markov) process $Q_{t}^{\oplus}$ such that when $Q_{0}^{\oplus} \in \mathcal{Q}^{(n)}$, it is generated by the state vector $P_{n} \Psi /\left\|P_{n} \Psi\right\|$, i.e., it is a Markov process $Q_{t}^{(n)}$ in $\mathcal{Q}^{(n)}$ generated by $\mathscr{L}_{P_{n} \Psi /\left\|P_{n} \Psi\right\|}^{(n)}$. The equivariance statement follows directly, since $\left\|P_{n} \Psi_{t}\right\|^{2}=\mathbb{P}_{t}\left(\mathcal{Q}^{(n)}\right)$ is invariant under the evolution generated by $H_{0}=\bigoplus_{n} H^{(n)}$.

\subsubsection{Tensor Products}

Given a finite sequence of Hilbert spaces $\mathscr{H}^{[1]}, \ldots, \mathscr{H}^{[n]}$ with POVMs $P^{[i]}$ on configuration spaces $\mathcal{Q}^{[i]}$, and for each $i$ a family of generators $\mathscr{L}^{[i]}=\mathscr{L}_{W_{i}}^{[i]}$ labeled by the density matrices on $\mathscr{H}^{[i]}$, there is a canonically constructed family of generators $\mathscr{L}^{\otimes}=\mathscr{L}_{W}^{\otimes}$, labeled by the density matrices on the tensor product $\mathscr{H}^{[1]} \otimes \cdots \otimes \mathscr{H}^{[n]}$. The corresponding process takes place in the Cartesian product $\mathcal{Q}=\mathcal{Q}^{[1]} \times \cdots \times \mathcal{Q}^{[n]}$. If every $\mathscr{L}_{W_{i}}^{[i]}$ is equivariant with respect to the density matrix $W_{i}$ on $\mathscr{H}^{[i]}$ and the Hamiltonian $H^{[i]}$, then $\mathscr{L}_{W}^{\oplus}$ is equivariant with respect to $W$ on $\mathscr{H}^{[1]} \otimes \cdots \otimes \mathscr{H}^{[n]}$ and $H=\sum_{i} \mathbf{1} \otimes \cdots \otimes H^{[i]} \otimes \cdots \otimes \mathbf{1}=\sum_{i} H_{i}$.

Here are the details. The POVM that naturally arises from the data is ${ }^{10}$

$$
P\left(d \boldsymbol{q}_{1} \times \cdots \times d \boldsymbol{q}_{n}\right)=P^{[1]}\left(d \boldsymbol{q}_{1}\right) \otimes \cdots \otimes P^{[n]}\left(d \boldsymbol{q}_{n}\right) .
$$

For any $q \in \mathcal{Q}$, let $\boldsymbol{q}_{i}$ denote its $i$-th component and let $\widehat{q}_{i}=\left(\boldsymbol{q}_{1}, \ldots, \boldsymbol{q}_{i-1}, \boldsymbol{q}_{i+1}, \ldots, \boldsymbol{q}_{n}\right)$. For every $i$ and $\widehat{q}_{i}$, define

$$
W_{i}\left(\widehat{q}_{i}\right)=\frac{\operatorname{tr}_{\neq i}\left(W P\left(d \boldsymbol{q}_{1} \times \cdots \times \mathcal{Q}^{[i]} \times \cdots \times d \boldsymbol{q}_{n}\right)\right)}{\operatorname{tr}\left(W P\left(d \boldsymbol{q}_{1} \times \cdots \times \mathcal{Q}^{[i]} \times \cdots \times d \boldsymbol{q}_{n}\right)\right)},
$$

where $\operatorname{tr}_{\neq i}$ is the partial trace over all factors except $\mathscr{H}^{[i]}$. This $W_{i}$ is the conditional density matrix, regarded as a function of the configuration $\widehat{q}_{i}$ of the other particles. Now

\footnotetext{
${ }^{10}$ The existence of the tensor product POVM is a consequence of Corollary 7 in Section 4.4 of [15].
} 
consider the process on $\mathcal{Q}$ according to which the $i$-th particle moves as prescribed by $\mathscr{L}_{W_{i}}^{[i]}$ while the other particles remain fixed. The generator of this process is

$$
\mathscr{L}_{i} \rho:=\left[\mathscr{L}_{W_{i}\left(\widehat{q}_{i}\right)}^{[i]} \rho\left(\cdot \mid \widehat{q}_{i}\right)\right] \rho_{\neq i}\left(d \widehat{q}_{i}\right)
$$

where $\rho_{\neq i}$ is the marginal distribution of $\widehat{Q}_{i}$ (i.e., $\rho$ integrated over $\boldsymbol{q}_{i}$ ) and $\rho\left(\cdot \mid \widehat{q}_{i}\right)$ is the conditional distribution of $\boldsymbol{Q}_{i}$ given $\widehat{Q}_{i}=\widehat{q}_{i}$; the square bracket is a function of $\widehat{q}_{i}$ and a measure in $d \boldsymbol{q}_{i}$. Now define $\mathscr{L}_{W}^{\otimes} \rho=\sum_{i} \mathscr{L}_{i} \rho$.

To see that $\mathscr{L}^{\otimes}$ is equivariant when the $\mathscr{L}^{[i]}$ are, we have to check (91). Note first that $\mathbb{P}^{W}\left(d \boldsymbol{q}_{i} \mid \widehat{q}_{i}\right)=\operatorname{tr}\left(W_{i}\left(\widehat{q}_{i}\right) P^{[i]}\left(d \boldsymbol{q}_{i}\right)\right)$. Due to the equivariance of $\mathscr{L}^{[i]}$, for $\rho=\mathbb{P}^{W}$ the square bracket in (97) equals $(2 / \hbar) \operatorname{Im} \operatorname{tr}\left(W_{i}\left(\widehat{q}_{i}\right) P^{[i]}\left(d \boldsymbol{q}_{i}\right) H^{[i]}\right)$, from which we obtain (91) for $\mathscr{L}_{i}$ and $H_{i}$ and hence for $\mathscr{L}^{\otimes}$ and $H$.

The definition of $\mathscr{L}^{\otimes}$ reproduces the many-particles Bohm law (11) with or without spin from the one-particle version (or, for distinguishable particles, from several different one-particle versions having different masses and spins). Similarly, it reproduces the many-particles Bohm-Dirac law (31) from the one-particle version.

\subsubsection{Second Quantization of the POVM}

Let $\mathcal{Q}^{(n)}$ denote the space of all subsets-with-multiplicities of $\mathcal{Q}^{(1)}$ having $n$ elements (counting in the multiplicities). $P^{(1)}$ naturally defines a POVM $P^{(1) \otimes n}$ on $\left(\mathcal{Q}^{(1)}\right)^{n}$ acting on $\mathscr{H}^{(1) \otimes n}$ by $P^{(1) \otimes n}\left(d \boldsymbol{q}_{1} \times \cdots \times d \boldsymbol{q}_{n}\right)=P^{(1)}\left(d \boldsymbol{q}_{1}\right) \otimes \cdots \otimes P^{(1)}\left(d \boldsymbol{q}_{n}\right)$, and a POVM $P^{(n)}$ on $\mathcal{Q}^{(n)}$ acting on $\mathscr{F}^{(n)}=P_{ \pm} \mathscr{H}^{(1) \otimes n}$ (the $n$-particle sector of Fock space, with $P_{ \pm}$ the projection to the subspace of (anti-)symmetric elements of $\mathscr{H}^{(1) \otimes n}$, depending on whether we deal with fermions or bosons) by

$$
P^{(n)}(B)=P^{(1) \otimes n}\left\{\left(\boldsymbol{q}_{1}, \ldots, \boldsymbol{q}_{n}\right) \in\left(\mathcal{Q}^{(1)}\right)^{n}:\left\{\boldsymbol{q}_{1}, \ldots, \boldsymbol{q}_{n}\right\} \in B\right\}
$$

for $B \subseteq \mathcal{Q}^{(n)}$, where $\left\{\boldsymbol{q}_{1}, \ldots, \boldsymbol{q}_{n}\right\}$ should be understood as a set-with-multiplicities. ${ }^{11}$ Since $P^{(n)}(B)$ is invariant under permutations, it maps symmetric to symmetric and antisymmetric to anti-symmetric elements of $\mathscr{H}^{(1) \otimes n}$ and thus acts on $\mathscr{F}^{(n)}$ for bosonic or fermionic Fock space. ${ }^{12}$ The corresponding POVM on $\mathcal{Q}$ is then $P=\Gamma P^{(1)}=\bigoplus_{n} P^{(n)}$; more precisely, for $B \subseteq \mathcal{Q}$,

$$
P(B)=\bigoplus_{n=0}^{\infty} P^{(n)}\left(B \cap \mathcal{Q}^{(n)}\right)
$$

\footnotetext{
${ }^{11}$ This agrees with the definition given in Section 3.1 for the case of a PVM and the coincidence configurations removed from configuration space.

${ }^{12}$ In case that $P^{(1)}$ is nonatomic, $P^{(n)}$ can equivalently be defined in the following way: For the set $\Delta$ of coincidence configurations we set $P^{(n)}(\Delta)=0$, and for volumes $d \boldsymbol{q}_{1}, \ldots, d \boldsymbol{q}_{n}$ in $\mathcal{Q}^{(1)}$ that are pairwise disjoint, we have a corresponding volume $d q$ in $\mathcal{Q}^{(n)}$, which can be obtained from $d \boldsymbol{q}_{1} \times \cdots \times d \boldsymbol{q}_{n} \subseteq$ $\left(\mathcal{Q}^{(1)}\right)^{n}$ by forgetting the ordering, and we set $P^{(n)}(d q)=n ! P_{ \pm} P^{(1)}\left(d \boldsymbol{q}_{1}\right) \otimes \cdots \otimes P^{(1)}\left(d \boldsymbol{q}_{n}\right) P_{ \pm}$.
} 


\subsubsection{Construction of the Free Process}

Equipped with the two procedures for direct sums and tensor products, we complete the construction of the free process.

The "tensor product" procedure above provides a process on $\left(\mathcal{Q}^{(1)}\right)^{n}$ from $n$ identical copies of $\mathscr{L}^{(1)}$. For a state vector $\Psi^{(n)} \in \mathscr{F}^{(n)}=P_{ \pm} \mathscr{H}^{(1) \otimes n}$ from either the symmetric or the anti-symmetric elements of the $n$-fold tensor product space, let $W$ be the projection to $\Psi^{(n)}$; the generator $\mathscr{L}_{W}^{\otimes}$ is permutation invariant because the tensor-product construction of $\mathscr{L}_{W}^{\otimes}$ is permutation covariant and a permutation can at most change the state vector by a minus sign, which does not affect the density matrix. Consequently, the ordering of the configuration is irrelevant and may be ignored. We thus obtain a process on $\mathcal{Q}^{(n)}$ whose generator we call $\mathscr{L}^{(n)}$. We now apply the "direct sum" procedure to obtain a process on $\mathcal{Q}$.

\section{Towards a Notion of Minimal Process}

In this section, we investigate the common traits of the Markov processes relevant to Bell-type QFT, which can be summarized in the notion of a minimal process associated with $\Psi, H$, and $P$. We begin with a closer study of the minimal free generator (32), and then explain why we call the minimal jump rates "minimal." Finally, in Section 5.3, we give an outlook on the notion of minimal process.

\subsection{Free Process From Differential Operators}

In this section, we discuss some of the details, concerning the two equivalent formulas (32) and (35) for the backward and forward version of the minimal free generator in terms of $H, P$, and $\Psi$, that we omitted in Section 2.9. To begin with, $L$ as defined by (32) satisfies some necessary conditions for being a backward generator: $L f(q)$ is real, and $L \mathbf{1}=0$ where $\mathbf{1}$ is the constant 1 function (this corresponds to $\mathscr{L} \rho(\mathcal{Q})=0$, or conservation of total probability). In case $L$ is indeed a backward generator, the corresponding process is equivariant because

$$
\mathscr{L} \mathbb{P}(d q) \stackrel{\text { 355 }}{=} \operatorname{Re}\left\langle\Psi\left|\hat{\mathbf{1}} \frac{i}{\hbar}[H, P(d q)]\right| \Psi\right\rangle=\frac{2}{\hbar} \operatorname{Im}\langle\Psi|P(d q) H| \Psi\rangle \stackrel{26]}{=} \dot{\mathbb{P}}(d q) .
$$

One way to arrive at formula (32) has been described in Section [2.9, A different way, leading to (35), is to start from the ansatz $\mathscr{L} \rho=A \frac{d \rho}{d \mathbb{P}}$ where $A$ denotes a (signed-measure-valued) linear operator acting on functions. Equivariance means $A \mathbf{1}(d q)=\left\langle\Psi\left|\frac{i}{\hbar}[H, P(d q)]\right| \Psi\right\rangle$. This suggests $A f(d q)=\left\langle\Psi\left|\hat{f} \frac{i}{\hbar}[H, P(d q)]\right| \Psi\right\rangle$, or $A f(d q)=\left\langle\Psi\left|\frac{i}{\hbar}[H, P(d q)] \hat{f}\right| \Psi\right\rangle$, or a convex combination thereof. Since $A f(d q)$ must be real, we are forced to choose the combination with coefficients $\frac{1}{2}$ and $\frac{1}{2}$, or equivalently $A f(d q)=\operatorname{Re}\left\langle\Psi\left|\hat{f} \frac{i}{\hbar}[H, P(d q)]\right| \Psi\right\rangle$, which is (35).

That $\mathscr{L}$ generates a deterministic process (when it is a generator at all) is suggested by the following consideration - at least when $H$ and $P$ are time-reversal invariant: 
replacing $\Psi$ in (35) by $T \Psi$ where $T$ is the anti-linear time reversal operator (see Section 6.1) changes the sign of $\mathscr{L}$. The only generators $\mathscr{L}$ such that $-\mathscr{L}$ is also a generator are, presumably, those corresponding to deterministic motion.

This gives us an opportunity to check for which $H$ (32) does define a process: for a deterministic process we must have $L=v \cdot \nabla$ where $v$ is the velocity vector field. It is known that vector fields, understood as first-order differential operators, are those linear operators $L$ on the space of smooth functions that satisfy the Leibniz rule $L(f g)=$ $f L g+g L f$. (32) is certainly linear in $f$, so we have to check the Leibniz rule to see whether $L$ is indeed of the form $v \cdot \nabla$ and thus the backward generator of a process.

We can see no reason why $L$ should satisfy a Leibniz rule unless $P$ is a PVM, which implies that

$$
\hat{f} P(d q)=f(q) P(d q)
$$

and $H$ is such that for all (nice) functions $f$ and $g$,

$$
[[H, \hat{f}], \hat{g}]=\hat{h}
$$

for some function $h$, which holds if $H$ is a differential operator of order $\leq 2$. (If $H=-\Delta$, then $h=-2 \nabla f \cdot \nabla g$; if $H=-i \boldsymbol{\alpha} \cdot \nabla$ for whatever vector of matrices $\boldsymbol{\alpha}$, or if $H$ is a multiplication operator, then $h=0$.) To check that the Leibniz rule is obeyed in this case, note that we then have that $[H, \widehat{f g}]=[H, \hat{f} \hat{g}]=[H, \hat{f}] \hat{g}+\hat{f}[H, \hat{g}]=$ $\hat{f}[H, \hat{g}]+\hat{g}[H, \hat{f}]+[[H, \hat{f}], \hat{g}]$. Using this in (32), we find that, due to (198), the first two terms give the Leibniz rule, whereas the last term, due to (99), does not contribute to the real part in (32).

When $\mathscr{H}$ is an $L^{2}$ space over $\mathcal{Q}$ and $P$ the natural PVM, i.e., when $\Psi$ is a function, (32) can be written in the form

$$
L f(q)=\frac{1}{\hbar} \operatorname{Im} \frac{\Psi^{*}(q)([\hat{f}, H] \Psi)(q)}{\Psi^{*}(q) \Psi(q)}
$$

where $\hat{f}$ is the multiplication operator corresponding to $f$. From this, one easily reads off the Bohm velocity (11) for the $N$-particle Schrödinger operator (3) with or without spin. Similarly, we get the Bohm-Dirac theory when $H$ is the Dirac operator in $\mathscr{H}=$ Anti $L^{2}\left(\mathbb{R}^{3}, \mathbb{C}^{4}\right)^{\otimes N}, \mathcal{Q}$ the manifold of subsets of $\mathbb{R}^{3}$ with $N$ elements, and $P$ the obvious PVM. (100) also leads to the Bohm-Dirac motion if $\mathscr{H}=L^{2}\left(\mathbb{R}^{3}, \mathbb{C}^{4}\right)^{\otimes N}, \mathcal{Q}=\mathbb{R}^{3 N}$, and $P$ is the natural PVM, but not if $\mathscr{H}$ is the positive energy subspace because then the appropriate POVM $P$ is no longer a PVM.

To see that the "second quantization" algorithm maps minimal free generators to minimal free generators, or, in other words, preserves the relation (35) between Hamiltonian and generator, observe first that (35) naturally extends to density matrices, and the extension, if a generator, is equivariant. Next check that the "direct sum" and "tensor product" procedures of Section 4.2 are compatible with (35) when $P$ is a PVM. Finally, observe that the (anti-)symmetrization operator commutes with the $n$-particle Hamiltonian, with $P(B)$ for every permutation invariant set $B \subseteq\left(\mathcal{Q}^{(1)}\right)^{n}$, and with $\hat{f}$ for every permutation invariant function $f:\left(\mathcal{Q}^{(1)}\right)^{n} \rightarrow \mathbb{R}$. 


\subsection{Minimality}

In this section we explain in what sense the minimal jump rates (29) - or (22) - are minimal. In so doing, we will also explain the significance of the quantity $\mathbb{J}$ defined in (28), and clarify the meaning of the steps taken in Sections 2.6 and 2.7 to arrive at the jump rate formulas.

Given a Markov process $Q_{t}$ on $\mathcal{Q}$, we define the net probability current $j_{t}$ at time $t$ between sets $B$ and $B^{\prime}$ by

$$
\begin{aligned}
j_{t}\left(B, B^{\prime}\right)=\lim _{\Delta t \searrow 0} \frac{1}{\Delta t} & {\left[\operatorname{Prob}\left\{Q_{t} \in B^{\prime}, Q_{t+\Delta t} \in B\right\}-\right.} \\
& \left.-\operatorname{Prob}\left\{Q_{t} \in B, Q_{t+\Delta t} \in B^{\prime}\right\}\right] .
\end{aligned}
$$

This is the amount of probability that flows, per unit time, from $B^{\prime}$ to $B$ minus the amount from $B$ to $B^{\prime}$. For a pure jump process, we have that

$$
j_{t}\left(B, B^{\prime}\right)=\int_{q^{\prime} \in B^{\prime}} \sigma_{t}\left(B \mid q^{\prime}\right) \rho_{t}\left(d q^{\prime}\right)-\int_{q \in B} \sigma_{t}\left(B^{\prime} \mid q\right) \rho_{t}(d q),
$$

so that

$$
j_{t}\left(B, B^{\prime}\right)=j_{\sigma, \rho}\left(B \times B^{\prime}\right)
$$

where $j_{\sigma, \rho}$ is the signed measure, on $\mathcal{Q} \times \mathcal{Q}$, given by the integrand of (15),

$$
j_{\sigma, \rho}\left(d q \times d q^{\prime}\right)=\sigma\left(d q \mid q^{\prime}\right) \rho\left(d q^{\prime}\right)-\sigma\left(d q^{\prime} \mid q\right) \rho(d q) .
$$

For minimal jump rates $\sigma$, defined by (29) or (22) (and with the probabilities $\rho$ given by (24), $\rho=\mathbb{P})$, this agrees with (28), as was noted earlier,

$$
j_{\sigma, \rho}=\mathbb{J}_{\Psi, H, P}
$$

where we have made explicit the fact that $\mathbb{J}$ is defined in terms of the quantum entities $\Psi, H$, and $P$. Note that both $\mathbb{J}$ and the net current $j$ are anti-symmetric, $\mathbb{J}^{\text {tr }}=-\mathbb{J}$ and $j^{\text {tr }}=-j$, the latter by construction and the former because $H$ is Hermitian. (Here $\operatorname{tr}$ indicates the action on measures of the transposition $\left(q, q^{\prime}\right) \mapsto\left(q^{\prime}, q\right)$ on $\mathcal{Q} \times \mathcal{Q}$.) The property (105) is stronger than the equivariance of the rates $\sigma, \mathscr{L}_{\sigma} \mathbb{P}_{t}=d \mathbb{P}_{t} / d t$ : Since, by (15),

$$
\left(\mathscr{L}_{\sigma} \rho\right)(d q)=j_{\sigma, \rho}(d q \times \mathcal{Q})
$$

and, by (28),

$$
\frac{d \mathbb{P}}{d t}(d q)=\mathbb{J}(d q \times \mathcal{Q})
$$

the equivariance of the jump rates $\sigma$ amounts to the condition that the marginals of both sides of (105) agree,

$$
j_{\sigma, \rho}(d q \times \mathcal{Q})=\mathbb{J}(d q \times \mathcal{Q}) .
$$


In other words, what is special about processes with rates satisfying (105) is that not only the single-time distribution but also the current is given by a standard quantum theoretical expression in terms of $H, \Psi$, and $P$. That is why we call (105) the standardcurrent property - defining standard-current rates and standard-current processes.

Though the standard-current property is stronger than equivariance, it alone does not determine the jump rates, as already remarked in [2, 30]. This can perhaps be best appreciated as follows: Note that (104) expresses $j_{\sigma, \rho}$ as twice the anti-symmetric part of the (nonnegative) measure

$$
C\left(d q \times d q^{\prime}\right)=\sigma\left(d q \mid q^{\prime}\right) \rho\left(d q^{\prime}\right)
$$

on $\mathcal{Q} \times \mathcal{Q}$ whose right marginal $C\left(\mathcal{Q} \times d q^{\prime}\right)$ is absolutely continuous with respect to $\rho$. Conversely, from any such measure $C$ the jump rates $\sigma$ can be recovered by forming the Radon-Nikodým derivative

$$
\sigma\left(d q \mid q^{\prime}\right)=\frac{C\left(d q \times d q^{\prime}\right)}{\rho\left(d q^{\prime}\right)} .
$$

Thus, given $\rho$, specifying $\sigma$ is equivalent to specifying such a measure $C$.

In terms of $C$, the standard-current property becomes (with $\rho=\mathbb{P}$ )

$$
2 \text { Anti } C=\mathbb{J} .
$$

Since (recalling that $\mathbb{J}=\mathbb{J}^{+}-\mathbb{J}^{-}$is anti-symmetric)

$$
\mathbb{J}=2 \text { Anti } \mathbb{J}^{+},
$$

an obvious solution to (111) is

$$
C=\mathbb{J}^{+},
$$

corresponding to the minimal jump rates. However, (105) fixes only the anti-symmetric part of $C$. The general solution to (111) is of the form

$$
C=\mathbb{J}^{+}+S
$$

where $S\left(d q \times d q^{\prime}\right)$ is symmetric, since any two solutions to (111) have the same antisymmetric part, and $S \geq 0$, since $S=C \wedge C^{\text {tr }}$, because $\mathbb{J}^{+} \wedge\left(\mathbb{J}^{+}\right)^{\text {tr }}=0$.

In particular, for any standard-current rates, we have that

$$
C \geq \mathbb{J}^{+}, \quad \text { or } \quad \sigma\left(d q \mid q^{\prime}\right) \geq \frac{\mathbb{J}^{+}\left(d q \times d q^{\prime}\right)}{\mathbb{P}\left(d q^{\prime}\right)} .
$$

Thus, among all jump rates consistent with the standard-current property, one choice, distinguished by equality in (114), has the least frequent jumps, or the smallest amount of stochasticity: the minimal rates (29). 


\subsection{Minimal Processes}

We have considered in this paper minimal jump processes, i.e., jump processes with rates (29), associated with integral operators $H$. There is a more general notion of minimal process, such that there is a minimal process associated with every Hamiltonian from a much wider class than that of integral operators; a class presumably containing all Hamiltonians relevant to QFT. This will be discussed in detail in a forthcoming work [16].

Bohmian mechanics is, in this sense, the minimal process associated with the Schrödinger Hamiltonian (3). The minimal process associated with an integral operator is the jump process with minimal rates. When the minimal free generator (32) exists, i.e., when (32) is a generator, it generates the minimal process associated with $H$. The minimal process associated with the Hamiltonian of a QFT is the one we have obtained in this paper by means of process additivity. The concept of minimal process directly provides, perhaps always, the process relevant to a Bell-type QFT.

To begin to convey the notion of the minimal process, we generalize the standardcurrent property (cf. Section 5.2) from pure jump processes to general Markov processes: the net probability current $j$ of a Markov process defines a bilinear form

$$
j_{t}(f, g)=\lim _{\Delta t \searrow 0} \frac{1}{\Delta t} \mathbb{E}\left(f\left(Q_{t+\Delta t}\right) g\left(Q_{t}\right)-f\left(Q_{t}\right) g\left(Q_{t+\Delta t}\right)\right)=\left(g, L_{t} f\right)-\left(f, L_{t} g\right)
$$

where $L_{t}$ is its backward generator, and $($,$) on the right hand side means the scalar$ product of $L^{2}\left(\mathcal{Q}, \rho_{t}\right)$. Then the Markov process satisfies the standard-current property if $\rho_{t}=\mathbb{P}_{t}$ and (for $f$ and $g$ real) $j_{t}(f, g)$ is equal to

$$
\mathbb{J}_{t}(f, g)=\frac{2}{\hbar} \operatorname{Im}\left\langle\Psi_{t}|\hat{f} H \hat{g}| \Psi_{t}\right\rangle,
$$

or, in other words, if twice the anti-symmetric part of its backward generator $L_{t}$ agrees with the operator corresponding to $\mathbb{J}_{t}$ as given by $\left(\mathbb{J}_{t} f, g\right)=\mathbb{J}_{t}(f, g), 2$ Anti $L_{t}=\mathbb{J}_{t}$. The minimal process is then the standard-current process that has, in a suitable sense, the smallest amount of randomness.

Let us consider some examples. The diffusion process with generator $\mathscr{L}$ given below (and for $\rho=\mathbb{P}$ ) has the standard-current property (in fact, because its "current velocity" [26] is $v$ ) for the Schrödinger Hamiltonian (3) but is not minimal:

$$
\mathscr{L} \rho=\frac{\lambda}{2} \Delta \rho-\operatorname{div}(\rho \tilde{v}), \text { with } \tilde{v}:=v+\frac{\lambda}{2} \nabla\left(\log |\Psi|^{2}\right)
$$

where $\lambda$ is any positive constant (the diffusion constant) and $v$ is the Bohmian velocity (11); this process was already considered in [24, 10]. Note that Nelson's stochastic mechanics [26] corresponds to $\lambda=\hbar$. It is obvious without any mathematical analysis that the smallest amount of stochasticity corresponds to absence of diffusion, $\lambda=0$, which yields Bohmian mechanics. Processes like the diffusion (117) for $\lambda>0$ seem less natural for the fundamental evolution law of a physical theory since they involve greater mathematical complexity than is needed for a straightforward association of a process 
with $H$ and $\Psi$. Examples of processes that do not have the standard-current property, for the Schrödinger Hamiltonian (3), are provided by the alternative velocity formulas considered by Deotto and Ghirardi [12]; one can say that their current is not the one suggested by $H$ and $\Psi$.

We return to the general discussion of the minimal process. As we have already indicated, when, for a standard-current process, we view $\mathbb{J}$ as well as its backward generator $L$ as operators on $L^{2}(\mathcal{Q}, \mathbb{P})$, then $\frac{1}{2} \mathbb{J}$ is the anti-symmetric (skew-adjoint) part of $L$; thus, only the symmetric (self-adjoint) part of $L$ remains at our disposal. Since one of the properties of a backward generator is $L \mathbf{1}=0$, the first possibility $\tilde{L}$ for $L$ that may satisfy the formal criteria for being a backward generator is $\tilde{L} f=\frac{1}{2} \mathbb{d} f-\left(\frac{1}{2} \rrbracket \mathbf{1}\right) f$. When $P$ is a PVM, this is also the operator we obtain by applying, to an arbitrary quantum Hamiltonian $H$, the formula (32) for what we called the minimal free generator, which we repeat here for convenience:

$$
\tilde{L} f(q)=\operatorname{Re} \frac{\left\langle\Psi\left|P(d q) \frac{i}{\hbar}[H, \hat{f}]\right| \Psi\right\rangle}{\langle\Psi|P(d q)| \Psi\rangle}
$$

Whereas this formula merely provided an alternative definition of the free process in Section 2.9, it now plays a different role: a step towards obtaining the minimal process from the Hamiltonian $H$. As we have pointed out in Section 2.9, $\tilde{L}$ is also an obvious naive guess for the backward generator $L$, quite independent of equivariance or the current $\mathbb{J}$, since $\frac{i}{\hbar}[H, \hat{f}]$ is the time derivative of $\hat{f}$. Moreover, it manifestly satisfies $\tilde{L} \mathbf{1}=0$. For the backward generator $L$ of a standard-current process we must have, when $P$ is a PVM, that $L=\tilde{L}+S$ where $S$ is a symmetric operator and $S \mathbf{1}=0$. For the minimal process, we have to choose $S$ as small as possible-while keeping $S$ symmetric and $L$ a backward generator.

Suppose $P$ is a PVM. Observe then that if $H$ is a differential operator (as $H_{0}$ often is) of the kind considered in Section 2.9, $\tilde{L}$ is itself a backward generator, so that $S=0$ is a possible, and in fact the smallest, choice. If $H$ is an integral operator, what keeps $\tilde{L}$, an integral operator as well, from being a backward generator is that the off-diagonal part of its $\mathbb{P}$-kernel $\left(q, \tilde{L} q^{\prime}\right)=\mathbb{P}(q) \tilde{L}\left(q, q^{\prime}\right)=\frac{1}{\hbar} \operatorname{Im}\langle\Psi \mid q\rangle\left\langle q|H| q^{\prime}\right\rangle\left\langle q^{\prime} \mid \Psi\right\rangle$ may assume negative values whereas the off-diagonal part of the $\mathbb{P}$-kernel of $L,\left(q, L q^{\prime}\right)=\mathbb{P}(q) \sigma\left(q \mid q^{\prime}\right)$, cannot be negative. The smallest possible choice of $S$ has as off-diagonal elements what is needed to compensate the negative values, and this leads to the minimal jump process, as described in Section 5.2. The diagonal part contains only what is needed to ensure that $S \mathbf{1}=0$. For $H$ of the form $H_{0}+H_{I}$, the role of $S$ is again to compensate negative values off the diagonal, and the minimal process has velocities determined by $H_{0}$ via (32) and jump rates determined by $H_{I}$ via (29).

In any case, the backward generator of the minimal process is the one closest, in a suitable sense, to (118). This formula may thus be regarded as containing the essential structure of $L$, for the deterministic as well as for the jump part of the process.

Another approach towards a general notion of minimal process may be to approximate $H$ by Hilbert-Schmidt operators $H_{n}$, with which are associated, according to the results of Sections 4.2.1 and 4.2.4 of [15], minimal jump processes $Q_{n}$, and take the limit 
$n \rightarrow \infty$ of the processes $Q_{n}$. This leads to a number of mathematical questions, such as under what conditions on $H, \Psi, P$, and $H_{n}$ does a limiting process exist, and is it independent of the choice of the approximating sequence $H_{n}$.

\section{Remarks}

\subsection{Symmetries}

Process additivity preserves symmetries, in the sense that the process generated by $\sum \mathscr{L}^{(i)}$ shares the symmetries respected by all of the building blocks $\mathscr{L}^{(i)}$. This section elaborates on this statement, and the following ones: The minimal jump rates (29) and the minimal free generator (32) share the symmetries of the Hamiltonians with which they are associated. The "second quantization" algorithm preserves the symmetries respected by the one-particle process.

Here are some desirable symmetries that may serve as examples: space translations, rotations and inversion, time translations and reversal, Galilean or Lorentz boosts, global change of phase $\Psi \rightarrow e^{i \theta} \Psi$, relabeling of particles, ${ }^{13}$ and gauge transformations.

We focus first on symmetries that do not involve time in any way, such as rotation symmetry. In this case, a symmetry group $G$ acts on $\mathcal{Q}$, so that to every $g \in G$ there corresponds a mapping $\varphi^{g}: \mathcal{Q} \rightarrow \mathcal{Q}$. In addition, $G$ acts on $\mathscr{H}$ through a projective unitary (or anti-unitary) representation, so that to every $g \in G$ there corresponds a unitary (or anti-unitary) operator $U_{g}$. Then the theory is $G$-invariant if both the wave function dynamics and the process on $\mathcal{Q}$ are, i.e., if $H$ is $G$-invariant,

$$
U_{g}^{-1} H U_{g}=H
$$

and

$$
\varphi^{g}\left(Q_{t}^{\Psi}\right)=Q_{t}^{U_{g} \Psi}
$$

in distribution on path space. A necessary condition for (120) is that the "configuration observable" transforms like the configuration, in the sense that

$$
U_{g}^{-1} P(\cdot) U_{g}=\varphi_{*}^{g} P(\cdot),
$$

where $\varphi_{*}$ denotes the action of $\varphi$ on measures. Without (121), (120) would already fail at time $t=0$, no matter what the generator is. Given (121), (120) is equivalent to the $G$-invariance of the generator:

$$
\varphi_{*}^{g} \mathscr{L}^{\Psi} \varphi_{*}^{g^{-1}}=\mathscr{L}^{U_{g} \Psi}
$$

Since $\varphi_{*}^{g}$ is a linear operator, it follows immediately that the sum of $G$-invariant generators is again $G$-invariant. The minimal jump process, when it exists, is $G$-invariant,

\footnotetext{
${ }^{13}$ This may mean two things: changing the artificial labels given to identical particles, or exchanging two species of particles.
} 
as follows from the fact that $\varphi_{*}^{g} \sigma^{\Psi}\left(d q \mid \varphi^{g}\left(q^{\prime}\right)\right)=\sigma^{U_{g} \Psi}\left(d q \mid q^{\prime}\right)$, which can be seen by inspecting the jump rate formula (29). The minimal free generator (35) satisfies (122) by virtue of (119) and (121). "Second quantization" provides $G$-actions on $\Gamma \mathcal{Q}^{(1)}$ and $\mathscr{F}=\Gamma \mathscr{H}^{(1)}$ from given actions on $\mathcal{Q}^{(1)}$ and $\mathscr{H}^{(1)}$; (119), (121) and (122) are inherited from their 1-particle versions.

Time-translation invariance is particularly simple. Consider generators $\mathscr{L}_{\Psi}^{(i)}$ which do not depend on time except through their dependence on $\Psi$. Then the same is true of $\sum \mathscr{L}^{(i)}$. The same can be said of the "second quantized" generator, and, provided $H$ is time-independent, of the minimal jump rates (29) and the minimal free generator (35).

Next we consider time reversal. It is represented on $\mathscr{H}$ by an anti-unitary operator $T$, i.e., an anti-linear operator such that $\langle T \Phi \mid T \Psi\rangle$ is the conjugate of $\langle\Phi \mid \Psi\rangle$. We assume that the Hamiltonian is reversible, $T H T^{-1}=H$. Then the reversibility of the theory means that

$$
Q_{-t}^{\Psi}=Q_{t}^{T \Psi}
$$

in distribution on path space, where the superscript should be understood as indicating the state vector at $t=0$. The necessary condition analogous to (121) reads

$$
T^{-1} P(\cdot) T=P(\cdot)
$$

and given that, (123) is equivalent to the $T$-invariance of the generator:

$$
\overline{\mathscr{L}}_{\Psi}=\mathscr{L}_{T \Psi} \text {, or } \bar{L}_{\Psi}=L_{T \Psi},
$$

where $\overline{\mathscr{L}}$ and $\bar{L}$ denote the forward and backward generator of the time-reversed process. $\bar{L}$ can be computed from $L$, for an equivariant Markov process, according to ${ }^{14}$

$$
\bar{L} f=L^{\dagger} f-\left(L^{\dagger} \mathbf{1}\right) f
$$

\footnotetext{
${ }^{14}$ To make this formula plausible, it may be helpful to note that the second term on the right hand side is just the correction needed to ensure that $L^{\dagger} \mathbf{1}=0$, a necessary condition for being a backward generator. If $\mathbb{P}$ were stationary, the second term on the right hand side would vanish.

Here is a derivation of (126): Let $(f, g)=\int_{q \in \mathcal{Q}} f(q) g(q) \mathbb{P}(d q)$ be the scalar product in $L^{2}(\mathcal{Q}, \mathbb{P})$. It follows from the definition (8) of $L$ that

$$
(g, L f)=\lim _{t \searrow 0} \frac{1}{t} \mathbb{E}\left(g\left(Q_{0}\right) f\left(Q_{t}\right)-g\left(Q_{0}\right) f\left(Q_{0}\right)\right) .
$$
}

Correspondingly, $\bar{L}$ is characterized (for $f$ and $g$ real) by

$$
\begin{aligned}
(g, \bar{L} f) & =\lim _{t \searrow 0} \frac{1}{t} \mathbb{E}\left(g\left(Q_{0}\right) f\left(Q_{-t}\right)-g\left(Q_{0}\right) f\left(Q_{0}\right)\right)= \\
& =\lim _{t \searrow 0} \frac{1}{t} \mathbb{E}\left(g\left(Q_{0}\right) f\left(Q_{-t}\right)-g\left(Q_{-t}\right) f\left(Q_{-t}\right)\right)+ \\
& +\lim _{t \searrow 0} \frac{1}{t} \mathbb{E}\left(g\left(Q_{-t}\right) f\left(Q_{-t}\right)-g\left(Q_{0}\right) f\left(Q_{0}\right)\right)= \\
& =(f, L g)-\int_{q \in \mathcal{Q}} g(q) f(q) \dot{\mathbb{P}}(d q) \stackrel{\text { 10] }}{=}(L g, f)-(L(g f), \mathbf{1})=\left(g, L^{\dagger} f\right)-\left(f g, L^{\dagger} \mathbf{1}\right),
\end{aligned}
$$

which amounts to (126). 
where ${ }^{\dagger}$ denotes the adjoint operator on $L^{2}(\mathcal{Q}, \mathbb{P})$, with $\mathbb{P}$ given by (24). Since $\bar{L}$ is linear in $L$, condition (125) is preserved when adding (forward or backward) generators; it is also preserved under "second quantization." For a pure jump process, (125) boils down to

$$
\sigma^{\Psi}\left(d q \mid q^{\prime}\right)\left\langle\Psi\left|P\left(d q^{\prime}\right)\right| \Psi\right\rangle=\sigma^{T \Psi}\left(d q^{\prime} \mid q\right)\langle\Psi|P(d q)| \Psi\rangle,
$$

which is satisfied for the minimal jump rates, by inspection of (29). The minimal free generator (32) changes sign when replacing $\Psi$ by $T \Psi$, which means the velocity changes sign, as it should under time reversal (see Section 5.11).

Invariance under Galilean boosts is a more involved story, and as it is not considered as fundamental in physics anyway, we omit it here. Lorentz boosts are even trickier, since for more than just one particle, they even fail to map (simultaneous) configurations into (simultaneous) configurations. As a result, the problem of Lorentz invariance belongs in an altogether different league, which shall not be entered here.

\subsection{On the Notion of Reversibility}

It may appear, and it is in fact a widespread belief, that stochasticity is incompatible with time reversibility. We naturally view the past as fixed, and the future, in a stochastic theory, as free, determined only by innovations. Even Bell expressed such a belief [5. p. 177]. However, from the proper perspective the conflict disappears, and this perspective is to consider the path space (of the universe) and the probability measure thereon. If $t \mapsto Q_{t}$ is a history of a universe governed by a Bell-type QFT, then its time reverse, $t \mapsto Q_{-t}$, is again a possible path of this Bell-type QFT, though corresponding to a different initial state vector $T \Psi$ instead of $\Psi$, with $T$ the time reversal operator as discussed in Section 6.1. More than this, the distribution of the reversed path $t \mapsto Q_{-t}$ coincides with the probability measure on path space arising from $T \Psi .{ }^{15}$

It may also be helpful to think of how the situation appears when viewed from outside space-time: then the path $Q_{t}$ corresponds to the decoration of space-time with a pattern of world lines, and this pattern is random with respect to a probability measure on what corresponds to path space, namely the space of all possible decorations of space-time. Then time reversal is a mere reflection, and for a theory to be time reversible means the same as being invariant under this reflection: that we could have had as well the reflected probability measure, provided we had started with $T \Psi$ instead of $\Psi$.

To sum up, we would like to convey that the sense of reversibility for Markov processes indeed matches the sense of reversibility that one should expect from a physical theory.

\footnotetext{
${ }^{15}$ We can be more precise about the meaning of the measure on path space: as in Bohmian mechanics [19, its role "is precisely to permit definition of the word 'typical'." [5. p. 129] Consequently, the meaning of the reversibility property of the measures we just mentioned is that the time reverse of a history that is typical with respect to $\Psi$, is typical with respect to $T \Psi$.
} 


\subsection{Heisenberg Picture}

In (24), we have applied the Schrödinger picture, according to which the state vector evolves while the operators remain fixed. Eq. (24) and the reasoning following it can as well be translated to the Heisenberg picture where the state vector $\Psi$ is regarded as fixed and the operators $P_{t}(\cdot)$ as evolving. Thus, we could equivalently write

$$
\mathbb{P}_{t}(d q)=\left\langle\Psi\left|P_{t}(d q)\right| \Psi\right\rangle
$$

instead of (24). Similarly, $H_{0}$ and $H_{I}$ become time-dependent while their sum is constant. We often use an ambiguous notation like $\langle\Psi|P(d q)| \Psi\rangle$ and formula (29) since the formulas are equally valid in both pictures (and, for that matter, in the interaction picture).

Like the jump rate formula (29), the formula (32) for the minimal free generator is equally valid in the Heisenberg picture.

We further remark that in the Heisenberg picture, the following nice equation holds for a pure jump process with minimal rates when $P$ is a PVM:

$$
\operatorname{Prob}\left\{Q_{t+d t} \in d q, Q_{t} \in d q^{\prime}\right\}=\left\langle\Psi\left|\left\{P_{t+d t}(d q), P_{t}\left(d q^{\prime}\right)\right\}\right| \Psi\right\rangle^{+}
$$

for $d q \cap d q^{\prime}=\emptyset$, where $\{$,$\} on the right hand side means the anti-commutator. The$ similarity to the one-time distribution formula

$$
\operatorname{Prob}\left\{Q_{t} \in d q\right\}=\left\langle\Psi\left|P_{t}(d q)\right| \Psi\right\rangle
$$

is striking. Specifying the two-time distribution for infinitesimal time differences is a way of characterizing a Markov process, equivalent to specifying the (forward or backward) generator and the one-time distribution. Thus, for a PVM $P$ (128) provides another formula for the minimal jump rates (29). A similar formula for the process generated by the minimal free generator (32) is $\mathbb{E}\left(g\left(Q_{t}\right) f\left(Q_{t+d t}\right)\right)=\frac{1}{2}\left\langle\Psi\left|\left\{\hat{g}_{t}, \hat{f}_{t+d t}\right\}\right| \Psi\right\rangle$.

\subsection{Examples of Process Additivity}

Among different versions of Bohmian mechanics we find numerous examples of process additivity (and, remarkably, no example violating it):

- The Hamiltonian for $n$ noninteracting particles is the sum of the Hamiltonians for the individual particles, and it is easy to see that the vector field defining Bohmian mechanics for the $n$-particle system is the sum of the vectors fields (each regarded as vectors fields on $\mathbb{R}^{3 n}$ ) for the particles. As already mentioned, sums of generators for deterministic processes amount to sums of the defining vector fields.

Moreover, the vector field for each particle is essentially the Bohmian one-particle law. To point out that this is a nontrivial fact, we mention that this is not so for the alternative velocity formula (10.2) in [12] considered by Deotto and Ghirardi, for which the velocity of the $i$-th particle differs from the one-particle law. So Bohmian mechanics of $n$ particles can be viewed as built from $n$ copies of the one-particle version, in fact by the "second quantization" algorithm of Section 4.2 . 
- The vector field of Bohmian mechanics for a single spinless particle may also be seen as arising in this way. If a Hamiltonian $H=-X^{2}$ is the negative square of an (incompressible) vector field (regarded as a first-order differential operator) $X=a(\boldsymbol{x}) \cdot \nabla$ on $\mathbb{R}^{3}$ (with $\nabla \cdot a=0$ ensuring formal self-adjointness of the square), then the simplest equivariant process associated with $H$ is given by the velocity vector field

$$
v=\frac{2}{\hbar} \operatorname{Im} \frac{a \cdot \nabla \Psi}{\Psi} a .
$$

The corresponding backward generator is $L=\frac{2}{\hbar} \operatorname{Im}\left(\frac{X \Psi}{\Psi}\right) X$. Now $-\frac{\hbar^{2}}{2} \Delta=$ $-\sum_{\alpha} X_{\alpha}{ }^{2}$ is the sum of 3 negative squares of vector fields $X_{\alpha}=\frac{\hbar}{\sqrt{2}} \partial / \partial x^{\alpha}$ corresponding to the individual degrees of freedom. The associated Bohm velocity is the sum of the velocities corresponding to the squares. So Bohmian mechanics in three dimensions can be viewed as built of 3 copies of the one-dimensional version. To point out that this is a nontrivial fact, we mention that this is not true, e.g., of the velocity formulas (10.1) and (10.2) in [12, which do not make sense in dimensions other than 3 .

- If we add an interaction potential $V$ to $-\frac{\hbar^{2}}{2} \Delta$, the Bohm velocity is the appropriate sum, since the operator $V$ is associated with the trivial motion $v=0$.

- We may also include an external vector potential $\boldsymbol{A}(\boldsymbol{x}, t)$ in the Schrödinger equation, that is, replace $-\frac{\hbar^{2}}{2} \Delta=-\frac{\hbar^{2}}{2} \nabla^{2}$ by $-\frac{\hbar^{2}}{2}\left(\nabla+i \frac{e}{\hbar} \boldsymbol{A}(\boldsymbol{x}, t)\right)^{2}=-\frac{\hbar^{2}}{2} \Delta-\frac{\hbar^{2}}{2}\left(i \frac{e}{\hbar} \nabla\right.$. $\left.\boldsymbol{A}+i \frac{e}{\hbar} \boldsymbol{A} \cdot \nabla\right)+\frac{e^{2}}{2} \boldsymbol{A}^{2}$. The sum of the associated velocities, namely

$$
\hbar \operatorname{Im} \frac{\Psi^{*} \nabla \Psi}{\Psi^{*} \Psi}+e \boldsymbol{A}+0
$$

equals the velocity one obtains directly, $\hbar \operatorname{Im} \Psi^{*}\left(\nabla+i \frac{e}{\hbar} \boldsymbol{A}\right) \Psi / \Psi^{*} \Psi$.

- In the Bohm-Dirac theory (31), however, one can include an external gauge connection $A_{\mu}(\boldsymbol{x}, t)$ in the Dirac equation without changing the velocity formula. That conforms with process additivity because the operator $\left(\gamma^{0}\right)^{-1} \gamma^{\mu} A_{\mu}=A_{0}+\boldsymbol{\alpha} \cdot \boldsymbol{A}$ is associated (termwise) with $v=0$.

- In the Dirac Hamiltonian $H=-i c \hbar \boldsymbol{\alpha} \cdot \nabla+\beta m c^{2}$, the first term corresponds to the Bohm-Dirac velocity (31), whereas the second term corresponds to $v=0$; as a consequence, the Bohm-Dirac velocity does not depend on the mass. Moreover, the three components of the Bohm-Dirac velocity are each equivariant with respect to the corresponding derivative term in $H$.

In addition, we point out cases of process additivity in the "second quantization" algorithm and minimal jump processes.

The "second quantized" generator $\Gamma \mathscr{L}^{(1)}$ as constructed in Section 4.2 provides an example of process additivity (or may be viewed as an application of process additivity):

$$
\mathscr{L}_{H_{0}, \Psi}=\sum_{n=0}^{\infty} \mathscr{L}_{H_{0}^{(n)}, \Psi^{(n)}},
$$


where the generators in the sum correspond to motions in the respective different sectors of $\mathcal{Q}$.

Suppose we regard the particles as ordered, $Q=\left(\boldsymbol{Q}_{1}, \ldots, \boldsymbol{Q}_{N}\right)$. Then another case of process additivity becomes visible:

$$
H_{0}^{(N)}=\sum_{i=1}^{N} h_{i}
$$

where $h_{i}$ is the one-particle Hamiltonian acting on the $i$-th particle. Correspondingly,

$$
\mathscr{L}_{H_{0}^{(N)}}=\sum_{i=1}^{N} \mathscr{L}_{i}
$$

where $\mathscr{L}_{i}$ is equivariant with respect to $h_{i}$. This applies not only to Bohmian mechanics (as described earlier in this section), but generally to the "second quantization" procedure as described in Section 4.2. We also note that the "second quantization" algorithm presented in Section 4.2 preserves process additivity in the sense that $\Gamma\left(\mathscr{L}_{1}^{(1)}+\mathscr{L}_{2}^{(1)}\right)=\Gamma\left(\mathscr{L}_{1}^{(1)}\right)+\Gamma\left(\mathscr{L}_{2}^{(1)}\right)$ while $\Gamma\left(H_{1}^{(1)}+H_{2}^{(1)}\right)=\Gamma\left(H_{1}^{(1)}\right)+\Gamma\left(H_{2}^{(1)}\right)$.

We now turn to process additivity among minimal jump processes.

A jump process generated by a sum need not be a minimal jump process even when its constituents are. But under certain conditions it is. Two such cases are the "direct sum" and "tensor product" processes constructed in Sections 4.2.1 and 4.2.2, $\mathscr{H}=\bigoplus_{n} \mathscr{H}^{(n)}$ with $\mathcal{Q}=\bigcup_{n} \mathcal{Q}^{(n)}$ and $H=\bigoplus_{n} H^{(n)}$, and $\mathscr{H}=\mathscr{H}^{[1]} \otimes \cdots \otimes \mathscr{H}^{[N]}$ with $\mathcal{Q}=\mathcal{Q}^{[1]} \times \cdots \times \mathcal{Q}^{[N]}$ and $H=\sum_{i} \mathbf{1} \otimes \cdots \otimes H^{[i]} \otimes \cdots \otimes \mathbf{1}$, with $\mathscr{L}=\sum \mathscr{L}_{i}$ where $\mathscr{L}_{i}$ acts nontrivially, in an obvious sense, only on $\mathcal{Q}^{(i)}$ or on $\mathcal{Q}^{[i]}$. These are special cases of the general fact that minimality is compatible with additivity whenever the addends of the Hamiltonian correspond to different sorts of jumps. That can be most easily understood in the case of a PVM corresponding to an orthonormal basis $\{|q\rangle: q \in \mathcal{Q}\}$ of $\mathscr{H}$ : suppose $H=H_{1}+H_{2}$ and for every pair $q, q^{\prime}$ either $\left\langle q\left|H_{1}\right| q^{\prime}\right\rangle=0$ or $\left\langle q\left|H_{2}\right| q^{\prime}\right\rangle=0$. Then $\sigma=\sigma_{1}+\sigma_{2}$. The corresponding condition in the POVM context is that the kernels of $H_{1}$ and $H_{2}$ have disjoint supports. When $H$ is naturally given as a sum this condition would be expected to be satisfied.

Finally, we remark that the minimal free generator $\mathscr{L}=\mathscr{L}^{H}$ as defined in (35) is additive in $H$.

\subsection{Second Quantization of a Minimal Jump Process}

We note that the "second quantization" of a minimal jump process associated with a PVM $P^{(1)}$, as described in Section 4.2, is the minimal jump process associated with the second-quantized Hamiltonian; this is a consequence of the observation that $\mathscr{L}_{i}$ generates the minimal jump process for $H_{i}$ in this case. This fact is probably physically irrelevant but it is mathematically nice. 


\subsection{Global Existence Question}

The rates $\sigma_{t}$ and velocities $v_{t}$, together with $\mathbb{P}_{t}$, define the process $Q_{t}$ associated with $H, P$, and $\Psi$, which can be constructed along the lines of Section 2.3. However, the rigorous existence of this process, like the global existence of solutions for an ordinary differential equation, is no trivial matter. See Section 4.3 of [15] for a discussion of what must be controlled in order to establish the global existence of the process, and [20] for an example of such a global existence proof.

\subsection{POVM Versus PVM}

As we have already remarked in footnote 4 , every POVM $P$ is related to a PVM $P_{\text {ext }}$, the Naimark extension, on a larger Hilbert space $\mathscr{H}_{\text {ext }}$ according to $P(\cdot)=P_{+} P_{\text {ext }}(\cdot) I$ with $P_{+}$the projection $\mathscr{H}_{\text {ext }} \rightarrow \mathscr{H}$ and $I$ the inclusion $\mathscr{H} \rightarrow \mathscr{H}_{\text {ext }}$. This fact allows a second perspective on $P$, and sometimes creates a certain ambiguity as to which process is the suitable one for a Bell-type QFT, as follows. At several places in this paper, we have described considerations leading to and methods for defining Markov processes, in particular minimal jump rates (29) and the minimal free generator (32); these considerations and methods could be applied using either $\mathscr{H}_{\text {ext }}$ and $P_{\text {ext }}$ or $\mathscr{H}$ and $P$. One would insist that the state vector $\Psi$ must lie in $\mathscr{H}$, the space of physical states, but even then one might arrive at different processes starting from $P$ or $P_{\text {ext }}$. To obtain a process from $P_{\text {ext }}$ requires, of course, that we have a Hamiltonian on $\mathscr{H}_{\text {ext }}$, while $H$ is defined on $\mathscr{H}$; such a Hamiltonian, however, can easily be constructed from $H$ by setting $H_{\text {ext }}=I H P_{+}$.

In some cases, the Naimark extension does not lead to an ambiguity. This is the case for the jump rate formula (29), since for $\Psi \in \mathscr{H},\left\langle\Psi\left|P_{\text {ext }}(d q)\right| \Psi\right\rangle=\langle\Psi|P(d q)| \Psi\rangle$ and $\left\langle\Psi\left|P_{\text {ext }}(d q) H_{\text {ext }} P_{\text {ext }}\left(d q^{\prime}\right)\right| \Psi\right\rangle=\left\langle\Psi\left|P(d q) H P\left(d q^{\prime}\right)\right| \Psi\right\rangle$. This fact suggests that, generally, the minimal process arising from $H_{\text {ext }}$ and $P_{\text {ext }}$ is the same as the one arising from $H$ and $P$.

The situation is different, however, when $H$ is defined on $\mathscr{H}_{\text {ext }}$ to begin with, and different from $H_{\text {ext }}$. This is the case with the free Dirac operator $h_{0}$, defined as a differential operator on $L^{2}\left(\mathbb{R}^{3}, \mathbb{C}^{4}\right)$, which differs from $P_{+} h_{0} P_{+}$. When we obtained in Section 2.9] the Bohm-Dirac motion (31) from the formula (32) for the minimal free generator, we used $h_{0}$ and $P_{\text {ext }}$. In contrast, the restriction of $h_{0}$ to the positive energy subspace, or equivalently $P_{+} h_{0} P_{+}$, possesses a kernel; more precisely, it is a convolution operator $S_{+} \star\left(h_{0} S_{+}\right) \star$ in the notation of Section 3.3 and thus corresponds to jumps. The associated minimal process on $\mathbb{R}^{3}$ presumably makes infinitely many jumps in every finite time interval, similar to the example of [15], Section 3.5.

Thus, there are two processes to choose between, the Bohm-Dirac motion and the minimal process for $P_{+} h_{0} P_{+}$. Both are equivariant, and thus it is arguably impossible to decide empirically which one is right. In our example theory in Section 3.3. we chose the simpler, deterministic one. But we leave to future work the discussion of which is more likely relevant to physics, and why. 


\subsection{The Role of Field Operators}

The Bell-type QFTs with which we have been concerned in this paper are models describing the behaviour of particles moving in physical 3-space, not of fields on 3-space. We have been concerned here mainly with a particle ontology, not a field ontology. This focus may be surprising at first: almost by definition, it would seem that QFT deals with fields, and not with particles. Consider only the occurrence (and prominence) of field operators in QFT!

But there is less to this than might be expected. The field operators do not function as observables in QFT. It is far from clear how to actually "observe" them, and even if this could somehow, in some sense, be done, it is important to bear in mind that the standard predictions of QFT are grounded in the particle representation, not the field representation: Experiments in high energy physics are scattering experiments, in which what is observed is the asymptotic motion of the outgoing particles. Moreover, for Fermi fields - the matter fields - the field as a whole (at a given time) could not possibly be observable, since Fermi fields anti-commute, rather than commute, at space-like separation. One should be careful here not to be taken in by the attitude widespread in quantum theory of intuitively regarding the operators as "quantities," as if they represented something out there in reality; see [9] for a critique of this attitude.

So let us focus on the role of the field operators in QFT. This seems to be to relate abstract Hilbert space to space-time: the field operators are attached to space-time points, unlike the quantum states $\Psi$, which are usually regarded not as functions but as abstract vectors. In orthodox quantum field theory the field operators are an effective device for the specification of Hamiltonians having good space-time properties. For our purposes here, what is critical is the connection between field operators and POVMs.

Throughout this paper, the connection between Hilbert space and the particle positions in physical space has been made through the POVM $P$, and through it alone. We now wish to emphasize that the field operators are closely related to $P$, and indeed that field operators are just what is needed for efficiently defining a POVM $P$ on $\Gamma\left(\mathbb{R}^{3}\right)$.

This connection is made through number operators $N(R), R \subseteq \mathbb{R}^{3}$. These define a number-operator-valued measure (NOVM) $N(\cdot)$ on $\mathbb{R}^{3}$, an "unnormalized POVM" $\left(N\left(\mathbb{R}^{3}\right)\right.$ is usually not the identity operator and $N(R)$ is usually an unbounded positive operator) for which the values $N(R)$ commute and are number operators: $\operatorname{spectrum}(N(R)) \subseteq\{0,1,2,3, \ldots\}$. (The basic difference, then, between a NOVM and a PVM is that the spectrum of the positive operators is $\{0,1,2,3, \ldots\}$ rather than just $\{0,1\}$.

There is an obvious one-to-one relation between NOVMs $N(\cdot)$ on $\mathbb{R}^{3}$ and PVMs $P$ on $\Gamma\left(\mathbb{R}^{3}\right)$, given by

$$
N(R)=\int_{q \in \Gamma\left(\mathbb{R}^{3}\right)} n_{R}(q) P(d q)
$$

where $n_{R}(q)=\#(q \cap R)$ is the number function on $\Gamma\left(\mathbb{R}^{3}\right)$ for the region $R$. Since (129) is the spectral decomposition of the commuting family $N(R)$, this correspondence is one-to-one. (Note that the joint spectrum of the commuting family $N(R)$ is the set of 
nonnegative-integer-valued measures $n_{R}$ on $\mathbb{R}^{3}$, one of the definitions of $\Gamma\left(\mathbb{R}^{3}\right)$ given in Section 2.8.)

The moral is that a NOVM on $\mathbb{R}^{3}$ is just a different way of speaking about a PVM $P$ on $\mathcal{Q}=\Gamma\left(\mathbb{R}^{3}\right)$. All other POVMs arise from PVMs by restriction to a subspace (Naimark's theorem [1] ). An easy way to obtain a NOVM $N$ starts with setting

$$
N(R)=\int_{R} \phi^{*}(\boldsymbol{x}) \phi(\boldsymbol{x}) d^{3} \boldsymbol{x}
$$

for suitable operators $\phi(\boldsymbol{x})$. An easy way to ensure that the $N(R)$ commute is to require that the operators $\phi(\boldsymbol{x})$ commute or anti-commute with each other and the adjoints $\phi^{*}\left(\boldsymbol{x}^{\prime}\right)$ for $\boldsymbol{x}^{\prime} \neq \boldsymbol{x}$. An easy way to ensure that the $N(R)$ have nonnegative integer eigenvalues is to require that

$$
\left[\phi(\boldsymbol{x}), \phi^{*}\left(\boldsymbol{x}^{\prime}\right)\right]_{ \pm}=\delta\left(\boldsymbol{x}-\boldsymbol{x}^{\prime}\right),
$$

where $[,]_{ \pm}$is the (anti-)commutator, and that there is a cyclic vacuum state $|0\rangle \in \mathscr{H}$ for which $\phi(\boldsymbol{x})|0\rangle=0$. The relations (131) are of course just the usual canonical (anti-)commutation relations that field operators are required to satisfy.

Moreover, in gauge theories the connection between matter field $\phi$ and the NOVM is perhaps even more compelling. Consider a gauge theory with internal state space $V$, equipped with the inner product $\langle\langle\cdot \mid \cdot\rangle\rangle$. Then, given $\boldsymbol{x} \in \mathbb{R}^{3}$, the matter field $\phi(\boldsymbol{x})$ should formally be regarded as a linear functional $V \rightarrow \mathcal{O}(\mathscr{H}), \xi \mapsto \phi_{\xi}(\boldsymbol{x})$, from the internal state space to operators on $\mathscr{H}$, with $\phi_{\xi^{*}}^{*}(\boldsymbol{x})=\left(\phi_{\xi}(\boldsymbol{x})\right)^{*}$ a linear function $V^{*} \rightarrow \mathcal{O}(\mathscr{H})$ on the dual of $V$. (131) then becomes $\left[\phi_{\xi}(\boldsymbol{x}), \phi_{\eta^{*}}^{*}\left(\boldsymbol{x}^{\prime}\right)\right]=\delta\left(\boldsymbol{x}-\boldsymbol{x}^{\prime}\right)\langle\langle\eta \mid \xi\rangle\rangle$. Thus the simplest gauge-invariant object associated with $\phi$ is the NOVM (130), with the integrand understood as the contraction of the tensor $V \times V^{*} \rightarrow \mathcal{O}(\mathscr{H}),(\xi, \eta) \mapsto$ $\phi_{\eta}^{*}(\boldsymbol{x}) \phi_{\xi}(\boldsymbol{x})$.

Hence, not only does the notion of particle not conflict with the prominence of field operators (see Sections 3.1 and 3.3 for explicit examples), but field operators have a natural place in a theory whose ultimate goal it is to govern the motion of particles. One of their important roles is to define the POVM $P$ that relates Hilbert space to configuration space. Quantum theory of fields or quantum theory of particles? A theory of particle motion exploiting field operators!

\section{Conclusions}

The essential point of this paper is that there is a direct and natural way of understanding QFT as a theory about moving particles, an idea pioneered, in the realm of nonrelativistic quantum mechanics, by de Broglie and Bohm. We leave open, however, three considerable gaps: the question of the process associated with the Klein-Gordon operator, the problem of removing cut-offs, and the issue of Lorentz invariance.

Acknowledgements. We thank James Taylor of Rutgers University and Stefan Teufel of Technische Universität München for helpful discussions. R.T. gratefully acknowledges 
support by the German National Science Foundation (DFG). N.Z. gratefully acknowledges support by INFN and DFG. Finally, we appreciate the hospitality that some of us have enjoyed, on more than one occasion, at the Mathematisches Institut of LudwigMaximilians-Universität München, at the Dipartimento di Fisica of Università di Genova, and at the Mathematics Department of Rutgers University.

\section{References}

[1] Ali, S.T., Emch, G.G.: Fuzzy Observables in Quantum Mechanics, J. Math. Phys. 15, 176-182 (1974)

[2] Bacciagaluppi, G., Dickson, M.: Dynamics for modal interpretations, Found. Phys. 29, 1165-1201 (1999), and quant-ph/9711048

[3] Bell, J.S.: Beables for quantum field theory, Phys. Rep. 137, 49-54 (1986). Reprinted in [5], p. 173.

[4] Bell, J.S.: De Broglie-Bohm, delayed-choice double-slit experiment, and density matrix, Int. J. Quant. Chem. 14, 155-159 (1980). Reprinted in [5], p. 111.

[5] Bell, J.S.: Speakable and unspeakable in quantum mechanics. Cambridge: Cambridge University Press (1987)

[6] Bohm, D.: A Suggested Interpretation of the Quantum Theory in Terms of "Hidden" Variables, I, Phys. Rev. 85, 166-179 (1952). Bohm, D.: A Suggested Interpretation of the Quantum Theory in Terms of "Hidden" Variables, II, Phys. Rev. 85, 180-193 (1952)

[7] Bohm, D., Hiley, B.J.: The Undivided Universe: An Ontological Interpretation of Quantum Theory. London: Routledge, Chapman and Hall (1993)

[8] Breiman, L.: Probability. Reading: Addison-Wesley (1968)

[9] Daumer, M., Dürr, D., Goldstein, S., Zanghì, N.: Naive Realism about Operators, Erkenntnis 45, 379-397 (1996), and quant-ph/9601013

[10] Davidson, M.: A generalization of the Fényes-Nelson stochastic model of quantum mechanics, Lett. Math. Phys. 3, 271-277 (1979)

[11] Davies, E.B.: Quantum Theory of Open Systems. London, New York, San Francisco: Academic Press (1976)

[12] Deotto, E., Ghirardi, G.C.: Bohmian mechanics revisited, Found. Phys. 28, 1-30 (1998), and quant-ph/9704021

[13] Dürr, D., Goldstein, S., Taylor, J., Tumulka, R., Zanghì, N.: Bosons, Fermions, and the Topology of Configuration Space, in preparation. 
[14] Dürr, D., Goldstein, S., Tumulka, R., Zanghì, N.: Trajectories and Particle Creation and Annihilation in Quantum Field Theory, J. Phys. A: Math. Gen. 36, 4143-4149 (2003), and quant-ph/0208072

[15] Dürr, D., Goldstein, S., Tumulka, R., Zanghì, N.: Quantum Hamiltonians and Stochastic Jumps, quant-ph/0303056

[16] Dürr, D., Goldstein, S., Tumulka, R., Zanghì, N.: Quantum Theory and Minimal Processes, in preparation.

[17] Dürr, D., Goldstein, S., Tumulka, R., Zanghì, N.: QED With Particles, in preparation.

[18] Dürr, D., Goldstein, S., Tumulka, R., Zanghì, N.: Trajectories From Klein-Gordon Functions, in preparation.

[19] Dürr, D., Goldstein, S., Zanghì, N.: Quantum equilibrium and the origin of absolute uncertainty, J. Statist. Phys. 67, 843-907 (1992)

[20] Georgii, H.-O., Tumulka, R.: Global Existence of Bell's Time-Inhomogeneous Jump Process for Lattice Quantum Field Theory, to appear in Markov Processes Rel. Fields (2004), and math.PR/0312294

[21] Goldstein, S.: Bohmian Mechanics (2001), in: Stanford Encyclopedia of Philosophy (Winter 2002 Edition), E.N. Zalta (ed.), http://plato.stanford.edu/archives/win2002/entries/qm-bohm/

[22] Guerra, F., Marra, R.: Discrete stochastic variational principles and quantum mechanics, Phys. Rev. D 29, 1647-1655 (1984)

[23] Haag, R.: Local Quantum Physics: Fields, Particles, Algebras. Berlin: SpringerVerlag (1992)

[24] Jaekel, M.T., Pignon, D.: Stochastic Processes of a Quantum State, Int. J. Theor. Phys. 24, 557-569 (1985)

[25] Kraus, K.: Position Observables of the Photon, p. 293-320 in W.C. Price and S.S. Chissick (eds.), The Uncertainty Principle and Foundations of Quantum Mechanics. New York: Wiley (1977)

[26] Nelson, E.: Quantum Fluctuations. Princeton: Princeton University Press (1985)

[27] Newton, T.D., Wigner, E.P.: Localized States for Elementary Systems, Rev. Mod. Phys. 21, 400-406 (1949)

[28] Norsen, T., Tumulka, R.: A Model of Photon Trajectories, in preparation.

[29] Reed, M., Simon, B.: Methods of Modern Mathematical Physics. I: Functional Analysis. New York and London: Academic Press (1972) 
[30] Roy, S.M., Singh, V.: Generalized beable quantum field theory, Phys. Lett. B 234, 117-120 (1990)

[31] Ruijsenaars, S.N.M.: Charged Particles in External Fields. I. Classical Theory, J. Math. Phys. 18 No 4, 720-737 (1977), and Charged Particles in External Fields. II. The Quantized Dirac and Klein-Gordon Theories, Commun. Math. Phys. 52, 267-294 (1977)

[32] Schweber, S.S.: An Introduction to Relativistic Quantum Field Theory. New York: Harper and Row (1961)

[33] Sudbery, A.: Objective interpretations of quantum mechanics and the possibility of a deterministic limit, J. Phys. A: Math. Gen. 20, 1743-1750 (1987)

[34] Vink, J.C.: Quantum mechanics in terms of discrete beables, Phys. Rev. A 48, 1808-1818 (1993) 